\title{
Une nouvelle preuve du théorème de point fixe de Handel
}

\author{
PATRICE LE CALVEZ
}

\begin{abstract}
M Handel has proved in [11] a fixed point theorem for an orientation preserving homeomorphism of the open unit disk, that may be extended to the closed disk and that satisfies a linking property of orbits. We give here a new proof of Handel's fixed point theorem, based on Brouwer theory and some plane topology arguments. We will slightly improve the theorem by proving the existence of a simple closed curve of index 1 . This index result was known to be true under an additional hypothesis and has been used by different authors (J Franks [9; 8], S Matsumoto [16]) to study homeomorphisms of surfaces.
\end{abstract}

37B20, 37C25, 37E30; 37E45, 37J10

\section{O Introduction}

M Handel a énoncé et prouvé dans [11] un théorème d'existence de point fixe pour un homéomorphisme $f$ préservant l'orientation du disque $\mathbf{D}=\{z \in \mathbb{C}|| z \mid<1\}$, qui se prolonge au bord et qui vérifie une propriété d'enlacement d'orbites qui s'énonce sous différentes formes, par exemple sous la forme suivante: il existe $n$ points $z_{i}$ dans $\mathbf{D}$ tels que

$$
\lim _{k \rightarrow-\infty} f^{k}\left(z_{i}\right)=\alpha_{i}, \lim _{k \rightarrow+\infty} f^{k}\left(z_{i}\right)=\omega_{i},
$$

où les $2 n$ points $\alpha_{i}$ et $\omega_{i}$ sont des points distincts de $S^{1}=\{z \in \mathbb{C}|| z \mid=1\}$ et où les droites $\Delta_{i}$ passant par $\alpha_{i}$ et $\omega_{i}$ délimitent un polygone convexe compact à $n$ côtés inclus dans D. Il s'agit d'un résultat d'une grande importance pour l'étude des homéomorphismes de surfaces, qui a maintes fois été utilisé dans le domaine (voir J Franks [9;8] ou S Matsumoto [16]). Dans la plupart des cas où ce théorème est utilisé, l'homéomorphisme $f$ vérifie une propriété supplémentaire qui permet d'obtenir une conclusion plus forte: il existe une courbe fermée simple $C \subset \mathbf{D}$ ne contenant pas de point fixe de $f$ et dont l'indice est 1. Rappelons que si $\Gamma: s \mapsto \Gamma(s)$ est un paramétrage de $C$ défini sur $S^{1}$, l'indice $i(f, C)$ est égal au degré de l'application

$$
s \mapsto \frac{f(\Gamma(s))-\Gamma(s)}{|f(\Gamma(s))-\Gamma(s)|} .
$$


Si $i(f, C) \neq 0$, on sait alors que $f$ a un point fixe dans la composante connexe de $\mathbf{D} \backslash C$ qui est simplement connexe.

La preuve de Handel de ce théorème de point fixe est considérée comme difficile. Elle utilise en particulier une généralisation de la classification de Nielsen-Thurston des homéomorphismes de surfaces, au cas du disque D privé d'une réunion finie d'orbites sans point limite. Elle utilise également la théorie de Brouwer des homéomorphismes du disque et une version homotopique de cette théorie. Nous donnons dans cet article une nouvelle preuve du théorème du point fixe de Handel qui n'utilise que la théorie de Brouwer "classique" et où n'apparaissent donc aucun argument géométrique (existence d'une métrique de courbure -1 , groupes fuchsiens,...). Même si la preuve n'est pas très courte, elle n'utilise, outre une partie de la théorie de Brouwer, que des arguments simples de topologie plane. Nous prouverons que les hypothèses du théorème impliquent l'existence d'une chaîne fermée de disques libres, c'est-à-dire d'une famille $\left(D_{i}\right)_{i \in \mathbb{Z} / r \mathbb{Z}}$ de parties ouvertes disjointes, connexes et simplement connexes, vérifiant $f\left(D_{i}\right) \cap D_{i}=\varnothing$, telle que $\bigcup_{k \geq 1} f^{k}\left(D_{i}\right) \cap D_{i+1} \neq \varnothing$, pour tout $i \in \mathbb{Z} / r \mathbb{Z}$. Le lemme de Franks [7] énonce qu'il existe alors une courbe fermée simple $C \subset \mathbf{D}$ ne contenant pas de point fixe de $f$ et dont l'indice est 1 . Ainsi le résultat d'indice est vrai sous les hypothèses du théorème du point fixe de Handel, il n'y a pas besoin d'ajouter des hypothèses. Nous verrons également que l'hypothèse de prolongement au bord peut être légèrement affaiblie.

Énonçons donc le théorème, dont les hypothèses sont illustrées sur la Figure 1:

Théorème $\mathbf{0 . 1}$ Soit $f$ un homéomorphisme de $\mathbf{D}$ qui préserve l'orientation et $n$ un entier supérieur ou égal à 3 . On suppose que les hypothèses suivantes sont satisfaites:

(i) il existe une famille $\left(z_{i}\right)_{i \in \mathbb{Z} / n \mathbb{Z}}$ dans $\mathbf{D}$ et deux familles $\left(\alpha_{i}\right)_{i \in \mathbb{Z} / n \mathbb{Z}}$ et $\left(\omega_{i}\right)_{i \in \mathbb{Z} / n \mathbb{Z}}$ dans $S^{1}$, telles que $\lim _{k \rightarrow-\infty} f^{k}\left(z_{i}\right)=\alpha_{i}$ et $\lim _{k \rightarrow+\infty} f^{k}\left(z_{i}\right)=\omega_{i}$, pour tout $i \in Z / n \mathbb{Z}$;

(ii) les $2 n$ points $\alpha_{i}, \omega_{i}, i \in \mathbb{Z} / n \mathbb{Z}$, sont distincts;

(iii) le seul, parmi ces points, qui se trouve sur l'intervalle ouvert du cercle orienté $S^{1}$ joignant $\omega_{i-1}$ à $\omega_{i}$ est $\alpha_{i+1}$;

(iv) $f$ se prolonge en un homéomorphisme de $\mathbf{D} \cup\left(\bigcup_{i \in \mathbb{Z} / n \mathbb{Z}}\left\{\alpha_{i}, \omega_{i}\right\}\right)$.

Il existe alors une courbe fermée simple $C \subset \mathbf{D}$ ne contenant pas de point fixe de $f$ et dont l'indice est 1. 


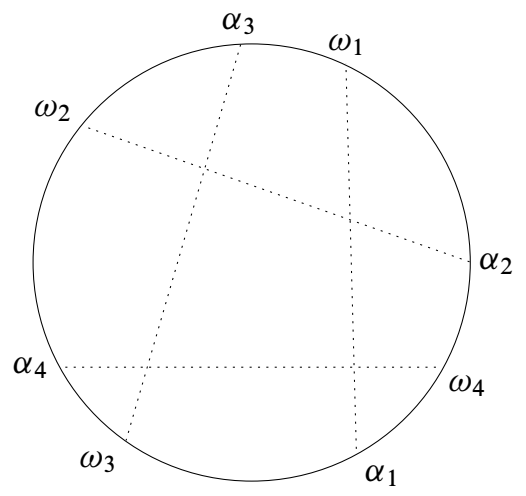

Figure 1

\section{Remarques}

(1) Le prolongement défini par (iv) fixe nécessairement les points $\alpha_{i}$ et $\omega_{i}$. Cette hypothèse de prolongement est indispensable. En effet, nous rappelerons dans la prochaine section que les orbites d'un homéomorphisme $f$ de $\mathbf{D}$, qui préserve l'orientation et qui n'a pas de point fixe, n'ont pas de point limite (en d'autres termes tendent vers le bout de $\mathbf{D}$ en $-\infty$ et $+\infty)$. Ainsi, si $n \geq 3$ est fixé, si $\left(z_{i}\right)_{i \in \mathbb{Z} / n \mathbb{Z}}$ est une famille arbitraire de points de $\mathbf{D}$ d'orbites distinctes, si $\left(\alpha_{i}\right)_{i \in \mathbb{Z} / n \mathbb{Z}}$ et $\left(\omega_{i}\right)_{i \in \mathbb{Z} / n \mathbb{Z}}$ sont deux familles arbitraires dans $S^{1}$ qui vérifient les hypothèses (ii) et (iii), il n'est pas difficile de construire un homéomorphisme $h$ de $\mathbf{D}$ préservant l'orientation, pour que l'hypothèse (i) soit satisfaite par la famille $\left(h\left(z_{i}\right)\right)_{i \in \mathbb{Z} / n \mathbb{Z}}$ et l'homéomorphisme $h \circ f \circ h^{-1}$. L'hypothèse de prolongement qui est supposée dans l'article original de Handel est un peu plus forte: $f$ se prolonge en un homéomorphisme de $\overline{\mathbf{D}}$.

(2) La propriété d'enlacement (ici l'hypothèse (iii)) peut être formulée différement. Ainsi, dans l'article de Handel, les $z_{i}$ sont indexés par $\{1, \ldots, n\}$, et l'hypothèse (iii) est remplacée par les deux assertions suivantes:

(iii') tout intervalle de $S^{1}$ délimité par deux points $\alpha$-limites (resp. $\omega$-limites) contient un point $\omega$-limite (resp. $\alpha$-limite);

(iii") pour tout $i \in\{1, \ldots, n\}$, il existe $i^{\prime} \in\{1, \ldots, n\}$ tel que $\alpha_{i}$ et $\omega_{i}$ sont séparés $\operatorname{sur} S^{1}$ par $\alpha_{i^{\prime}}$ et $\omega_{i^{\prime}}$.

Expliquons rapidement pourquoi ces deux conditions impliquent que la propriété (iii) est vraie (à orientation près) si on ne garde qu'un certain nombre des $z_{i}$. Notons $\Delta_{i}$ la droite affine réelle orientée, engendrée par le vecteur joignant $\alpha_{i}$ à $\omega_{i}$, et $\Pi_{i}^{+}$(resp. $\Pi_{i}^{-}$) le demi-plan affine situé strictement à gauche (resp. à droite) de $\Delta_{i}$. Quitte à 
perturber légèrement les points $\alpha_{i}$ et $\omega_{i}$, on peut supposer que l'intersection de trois droites $\Delta_{i}$ est toujours vide. Il suffit de trouver une partie $I$ de $\{1, \ldots, n\}$ telle que l'intersection $\bigcap_{i \in I} \Pi_{i}^{+}$ou $\bigcap_{i \in I} \Pi_{i}^{-}$soit relativement compacte dans $\mathbf{D}$, puis de choisir $I^{\prime} \subset I$, minimale parmi les parties de $I$ satisfaisant cette propriété. L'assertion (iii') implique que la fonction $v: \mathbb{C} \backslash \bigcup_{1 \leq i \leq n} \Delta_{i} \rightarrow \mathbb{N}$, qui à $z$ associe le nombre de demi-plans $\Pi_{i}^{+}$contenant $z$, prend exactement deux valeurs au voisinage de $S^{1}$ et que ces deux valeurs sont des entiers consécutifs. L'assertion (iii") implique qu'il existe au moins deux droites $\Delta_{i}$ et $\Delta_{i^{\prime}}$ qui s'intersectent dans D. Au voisinage du point d'intersection, la fonction $v$ prend trois valeurs consécutives. On en déduit que le maximum ou le minimum de la fonction $v$ sur $\mathbf{D} \backslash \bigcup_{1 \leq i \leq n} \Delta_{i}$ n'est pas l'une des deux valeurs près du bord. Dans le premier cas (resp. second cas), on considére une composante connexe $U$ de $\mathbf{D} \backslash \bigcup_{1 \leq i \leq n} \Delta_{i}$ où ce maximum (resp. minimum) est atteint. Elle est relativement compacte dans $\mathbf{D}$ et on peut écrire $U=\bigcap_{i \in I} \Pi_{i}^{+}$(resp. $\left.U=\bigcap_{i \in I} \Pi_{i}^{-}\right)$, où $I \subset\{1, \ldots, n\}$.

Nous allons continuer cette introduction en rappelant certaines applications importantes du théorème de Handel à l'étude des homéomorphismes de surfaces isotopes à l'identité Si $\left(F_{t}\right)_{t \in[0,1]}$ est une isotopie issue de l'identité sur une surface $M$, on peut définir la trajectoire $\gamma_{z}: t \mapsto F_{t}(z)$ de tout point $z \in M$. Si $z$ est un point périodique, de période $q \geq 1$, on obtient alors un lacet $\prod_{0 \leq i \leq q-1} \gamma_{F^{i}(z)}$ par assemblage des trajectoires, dont on note $\kappa\left(z_{i}\right) \in H_{1}(M, \mathbb{Z})$ la classe d'homologie. Les deux résultats qui suivent sont des conséquences directes du théorème de point fixe. Le premier est attribué par Franks dans [8] à M Betsvina et Handel, le second est dû à Franks [9]. Nous rappelerons également les grandes lignes des preuves que l'on trouve dans [9] et [8].

Théorème 0.2 Soit $M$ une partie ouverte de la sphère $S^{2}$, dont le complémentaire a au moins trois composantes connexes, et $\widetilde{M}$ son revêtement universel. Soit $\left(F_{t}\right)_{t \in[0,1]}$ une isotopie sur $M$ issue de l'identité, et $\left(f_{t}\right)_{t \in[0,1]}$ l'isotopie relevée à $\widetilde{M}$ qui est issue de l'identité. $S$ 'il existe un point périodique $z_{0}$, de période $q \geq 1$, tel que $\kappa\left(z_{0}\right)=0$, alors on peut trouver une courbe fermée simple $C \subset \widetilde{M}$ ne contenant pas de point fixe de $f_{1}$ et dont l'indice $i\left(f_{1}, C\right)$ est 1 . En particulier, $F_{1}$ a au moins un point fixe.

Idée de la preuve On peut munir $M$ d'une structure riemannienne complète de courbure -1 et identifier le revêtement universel $\widetilde{M}$ au disque de Poincaré D. On sait alors que $f_{1}$ se prolonge en un homéomorphisme de $\overline{\mathbf{D}}$ qui fixe tout point de $S^{1}$ (voir Handel et Thurston [12]). Notons $\Gamma$ l'unique géodésique fermée qui est librement homotope à $\prod_{0 \leq i \leq q-1} \gamma_{F_{1}^{i}\left(z_{0}\right)}$. Tout relèvement de $\Gamma$ dans $\mathbf{D}$ est une géodésique de $\mathbf{D}$ qui joint un point $\alpha \in S^{1}$ à un point $\omega \in S^{1}$. De plus, il existe un antécédent $\widetilde{z}_{0}$ de $z_{0}$ tel que $\lim _{k \rightarrow-\infty} f_{1}^{k}\left(z_{0}\right)=\alpha$ et $\lim _{k \rightarrow+\infty} f_{1}^{k}\left(z_{0}\right)=\omega$. Puisque $\Gamma$ est homologue à 
zéro dans $M$, il existe une fonction $\Lambda: M \backslash \Gamma \rightarrow \mathbb{Z}$, à support relativement compact, tel que $\Lambda\left(z^{\prime}\right)-\Lambda(z)$ représente le nombre d'intersection algébrique $\Gamma \wedge \Gamma^{\prime}$ entre $\Gamma$ et un arc quelconque $\Gamma^{\prime}$ qui joint $z$ à $z^{\prime}$. L'un au moins des deux nombres max $\Lambda$ ou $\min \Lambda$ doit être non nul. Supposons par exemple que max $\Lambda \neq 0$ et fixons une composante connexe $U$ de $M \backslash \Gamma$ où $\Lambda$ atteint son maximum. Il n'est pas difficile de voir que $U$ est un disque ouvert, que sa frontière est une courbe fermée simple, réunion de $p$ sous-segments de $\Gamma$, et que $U$ est localement à gauche de chacun de ces segments. Toute composante connexe de la préimage de $U$ dans $\mathbf{D}$ est l'intersection (relativement compacte) de $p$ demi-plans géodésiques, à gauche de géodésiques relevant $\Gamma$. Parmi ces demi-plans, on choisit une famille dont l'intersection est relativement compacte dans $\mathbf{D}$, et qui est minimale pour cette propriété. Les conditions du Théorème 0.1 sont alors vérifiées.

Théorème 0.3 Soit $M$ une surface compacte orientable de genre $g \geq 1$ et $\widetilde{M}$ son revêtement universel. Soit $\left(F_{t}\right)_{t \in[0,1]}$ une isotopie sur $M$ issue de l'identité et $\left(f_{t}\right)_{t \in[0,1]}$ l'isotopie relevée à $\widetilde{M}$ qui est issue de l'identité. On suppose qu'il existe $2 g+1$ points périodiques $z_{i}, 0 \leq i \leq 2 g$, tels que l'intérieur de l'enveloppe convexe des $\kappa\left(z_{i}\right) \in H_{1}(M, \mathbb{R})$ contient 0 . Il existe alors une courbe fermée simple $C \subset \widetilde{M}$ ne contenant pas de point fixe de $f_{1}$ et dont l'indice $i\left(f_{1}, C\right)$ est 1 . En particulier, $F_{1}$ a au moins deux points fixes.

Idée de la preuve Dans le cas où $g \geq 2$, la preuve est très proche de celle du Théorème 0.2. On munit $M$ d'une structure riemannienne de courbure -1 et on identifie $\widetilde{M}$ à $\mathbf{D}$. On sait, là encore, que $f_{1}$ se prolonge en un homéomorphisme de $\overline{\mathbf{D}}$ qui fixe tout point de $S^{1}$. On note $\Gamma_{i}$ la géodésique fermée homotope à $\prod_{0 \leq i \leq q_{i}-1} \gamma_{F_{1}^{i}\left(z_{0}\right)}$, où $q_{i}$ est la période de $z_{i}$. On peut alors trouver des entiers $m_{i}>0$ tels que $\sum_{i=0}^{2 g} m_{i} \kappa\left(z_{i}\right)=0$ La multi-courbe $\Gamma=\sum_{i=0}^{2 g} m_{i} \Gamma_{i}$ est homologue à zéro et on lui associe une fonction duale $\Lambda: M \backslash \Gamma \rightarrow \mathbb{Z}$, comme dans la preuve du Théorème 0.2 . Là encore, on peut supposer que max $\Lambda \neq 0$. Pour montrer le résultat d'indice, il suffit de prouver que toute composante connexe $U$ de $M \backslash \Gamma$ où $\Lambda$ atteint son maximum est un disque ouvert, puis de reprendre les arguments donnés dans la preuve précédente. Puisque les classes $\left[\Gamma_{i}\right]$ engendrent $H_{1}(M, \mathbb{R})$, l'application $\iota_{*}: H_{1}(U, \mathbb{R}) \rightarrow H_{1}(M, \mathbb{R})$ induite par l'inclusion $\iota: U \rightarrow M$ doit être nulle. On en déduit que l'intersection algébrique entre deux lacets quelconques de $U$ est nul et donc que $U$ est de genre zéro. Il reste à montrer que son complémentaire est connexe. Dans le cas contraire, il existerait une partition non triviale $\{0, \ldots, 2 g\}=I_{1} \sqcup I_{2}$, telle que $\sum_{i \in I_{1}} m_{i}\left[\Gamma_{i}\right]=\sum_{i \in I_{2}} m_{i}\left[\Gamma_{i}\right]=0$, ce qui contredirait le fait que 0 est à l'intérieur de l'enveloppe convexe des $\left[\Gamma_{i}\right]$.

Si $g=1$, la preuve est plus simple. On peut écrire $M=\mathbb{C} /(\mathbb{Z}+i \mathbb{Z})$ et on sait alors que $f_{1}$ commute avec les translations entières. On en déduit que $f_{1}-\operatorname{Id}_{\mathbb{C}}$ est 
une application bornée et donc que $f_{1}$ se prolonge en un homéomorphisme sur le compactifié de $\mathbb{C}$ obtenu en rajoutant les directions à l'infini. En d'autres termes, l'homéomorphisme $\tilde{f}_{1}=h \circ f_{1} \circ h^{-1}$ de $\mathbf{D}$, conjugué par $h: \mathbb{C} \rightarrow \mathbf{D}, z \mapsto \frac{z}{1+|z|}$, se prolonge en l'identité sur $S^{1}$. Remarquons maintenant que $\tilde{f}_{1}$ vérifie les hypothèses du Théorème 0.1. avec $n=3$.

Dans chacun des cas, on sait que $f_{1}$ (et donc que $F_{1}$ ) a au moins un point fixe. Si $F_{1}$ n'avait qu'un seul point fixe, la formule de Lefschetz nous dirait que son indice de Lefschetz est $2-2 g$. Tout point fixe de $f_{1}$ serait donc d'indice $2-2 g$, ce qui impliquerait que l'indice de toute courbe fermée simple $C$ ne contenant pas de point fixe de $f_{1}$ serait égal à $m(2-2 g) \leq 0$, l'entier $m$ étant le nombre de points fixes dans la composante connexe simplement connexe de $\widetilde{M} \backslash C$. Le même raisonnement, en utilisant cette fois-ci la formule de Lefschetz-Nielsen, nous dit en fait que $F_{1}$ a au moins deux points fixes qui se relèvent en des points fixes de $f_{1}$.

En utilisant alors un simple argument de perturbation, Franks donne dans [9] la version plus générale suivante, où l'ensemble de rotation désigne la partie convexe et compacte formée des vecteurs de rotation (ou enlacements asymptotiques) de toutes les mesures boréliennes de probabilité invariantes par $F_{1}$ (voir S Schwartzman [18]).

Théorème 0.4 Soit $M$ une surface compacte orientable de genre $g \geq 1$ et $\widetilde{M}$ son revêtement universel. Soit $\left(F_{t}\right)_{t \in[0,1]}$ une isotopie sur $M$ issue de l'identité et $\left(f_{t}\right)_{t \in[0,1]}$ l'isotopie relevée à $\widetilde{M}$ qui est issue de l'identité. Si l'ensemble de rotation $\rho\left(f_{1}\right) \subset H_{1}(M, \mathbb{R})$ défini par l'isotopie contient 0 dans son intérieur, il existe alors une courbe fermée simple $C \subset \widetilde{M}$ ne contenant pas de point fixe de $f_{1}$ et dont l'indice $i\left(f_{1}, C\right)$ est 1 .

Les résultats précédents sont d'une grande utilité pour montrer l'existence de points fixes ou de points périodiques. Intéressons nous par exemple au cas où $M$ est une surface compacte munie d'une forme symplectique et l'isotopie $\left(F_{t}\right)_{t \in[0,1]}$ est hamiltonienne (i.e. induite par un champ de vecteurs hamiltonien de classe $C^{1}$ dépendant du temps). Le vecteur de rotation de la mesure naturellement définie par la forme est alors égal à 0 . Un résultat, dû indépendamment à A Floer [5] et à J C Sikorav [19], nous dit que $F_{1}$ a au moins trois points fixes $z$ contractiles (i.e. tels que le lacet $\gamma_{z}$ soit homotope à zéro). Dans [9], Franks donne une preuve de ce résultat qui se déduit du Théorème 0.4, toujours par des arguments de perturbation. La méthode de Franks a été généralisée au cas des homéomorphismes. Matsumoto [16] utilise également le théorème de point fixe de Handel pour donner la version topologique suivante de la conjecture d'Arnold pour les homéomorphismes de surfaces: 
Théorème 0.5 Soit $M$ une surface compacte orientable de genre $g \geq 1$ et $\left(F_{t}\right)_{t \in[0,1]}$ une isotopie issue de l'identité. On suppose que $F_{1}$ préserve la mesure de Lebesgue $\mu$ et que le vecteur de rotation de cette mesure est nul. Il existe alors au moins trois points fixes contractiles.

Nous rappelerons dans la section qui suit certains points de la théorie des homéomorphismes du plan initiée par Brouwer. En particulier, nous donnerons des critères d'existence de courbes fermées simple d'indice 1 pour un homéomorphisme de $\mathbf{D}$ qui préserve l'orientation. Nous rappelerons dans la Section 2 la notion de décomposition en briques libre, qui sera l'outil principal pour montrer que ces critères sont vérifiés. Nous donnerons à la fin de la Section 2 le plan de la preuve du Théorème 0.1. Nous détaillerons cette preuve à partir de la Section 3 . Nous allons conclure cette introduction en introduisant les principales définitions de cet article.

On écrira respectivement $\bar{X}, \operatorname{Int}(X), \partial X$ pour l'adhérence, l'intérieur et la frontière d'une partie $X$ d'un espace topologique $E$. On dira qu'une partie $Y \subset E$ sépare deux parties connexes $X_{1}$ et $X_{2}$ si $Y$ est disjoint de $X_{1}$ et $X_{2}$ et si $X_{1}$ et $X_{2}$ sont dans des composantes connexes différentes de $E \backslash Y$.

On appelera disque ouvert (resp. disque fermé) d'une surface $M$ toute partie homéomorphe à $\mathbf{D}$ (resp. à $\overline{\mathbf{D}}$ ).

On appelera arc une application continue $\gamma$ d'un intervalle $I \subset \mathbb{R}$ vers une surface $M$, ou plus précisément, une classe d'équivalence d'applications par reparamétrage préservant l'orientation. On utilisera souvent, par abus de langage, le mot arc pour désigner l'image de l'arc, en faisant bien attention qu'il n'y ait pas d'ambiguité.

Si $\gamma_{1}: I_{1} \rightarrow M$ et $\gamma_{2}: I_{2} \rightarrow M$ sont deux arcs tels que

$$
\sup I_{1} \in I_{1}, \quad \inf I_{2} \in I_{2}, \quad \gamma_{1}\left(\sup I_{1}\right)=\gamma_{2}\left(\inf I_{2}\right),
$$

on notera $\gamma_{1} \gamma_{2}$ l'arc naturellement défini par assemblage de $\gamma_{1}$ et $\gamma_{2}$. Si $J$ est un intervalle de $\mathbb{Z}$ et si $\left(\gamma_{j}\right)_{j \in J}$ est une famille d'arcs $\gamma_{j}: I_{j} \rightarrow M$ telle que

$$
\sup I_{j} \in I_{j}, \quad \inf I_{j+1} \in I_{j+1}, \quad \gamma_{j}\left(\sup I_{j}\right)=\gamma_{j+1}\left(\inf I_{j+1}\right),
$$

si $j$ et $j+1$ appartiennent à $J$, on écrira $\prod_{j \in J} \gamma_{j}$ l'arc défini par assemblage des $\gamma_{j}$.

On appelera droite (resp. demi-droite négative, demi-droite positive, segment, cercle) d'une surface $M$ tout plongement topologique propre de $\mathbb{R}$ (resp. ]- $\infty, 0],[0,+\infty[$, $\left.[0,1], S^{1}\right)$, plus précisément, toute classe d'équivalence d'un tel plongement par reparamétrage préservant l'orientation. Un tel objet est alors déterminé par son image et une orientation. On utilisera, là encore, le mot droite, demi-droite, segment ou cercle, 
pour désigner l'image du plongement. Si $\gamma$ est un segment ou une demi-droite, on notera int $(\gamma)$ l'arc privé des ses extrémités.

On notera $\mathrm{Homeo}^{+}(\mathbf{D})$ l'ensemble des homéomorphismes du disque $\mathbf{D}$ qui préservent l'orientation. Pour tout $f \in \mathrm{Homeo}^{+}(\mathbf{D})$ on notera $\operatorname{Fix}(f)$ l'ensemble des points fixes de $f$. On dira qu'une partie $X \subset \mathbf{D}$ est libre si $f(X) \cap X=\varnothing$.

On identifiera $\mathbb{R}^{2}$ à $\mathbb{C}$, via l'isomorphisme naturel $(x, y) \mapsto x+i y$.

\section{Rappels sur la théorie de Brouwer}

Commençons par rappeler le lemme de translation de Brouwer qui est le point fondamental de la théorie (voir [1], [2], [4] ou [10]):

Proposition 1.1 Soit $f \in \mathrm{Homeo}^{+}(\mathbf{D})$. On peut trouver un cercle d'indice 1 dans chacun des deux cas suivants:

- il existe un segment $\gamma$ qui joint un point $z \in \operatorname{Fix}\left(f^{2}\right) \backslash \operatorname{Fix}(f)$ à $f(z)$ et tel que $f(\gamma) \cap \gamma=\{z, f(z)\}$;

- il existe un segment $\gamma$ qui joint un point $z \notin \operatorname{Fix}\left(f^{2}\right)$ à $f(z)$, tel que $f(\gamma) \cap \gamma=$ $\{f(z)\}$, et un entier $k \geq 2$ tel que $f^{k}(\gamma) \cap \gamma \neq \varnothing$.

Un segment $\gamma$ qui joint un point $z \notin \operatorname{Fix}(f)$ à $f(z)$ et tel que

$$
\begin{cases}f(\gamma) \cap \gamma=\{z, f(z)\} & \text { si } f^{2}(z)=z \\ f(\gamma) \cap \gamma=\{z\} & \text { si } f^{2}(z) \neq z,\end{cases}
$$

s'appelle un arc de translation de $f$ (voir Figure 2).
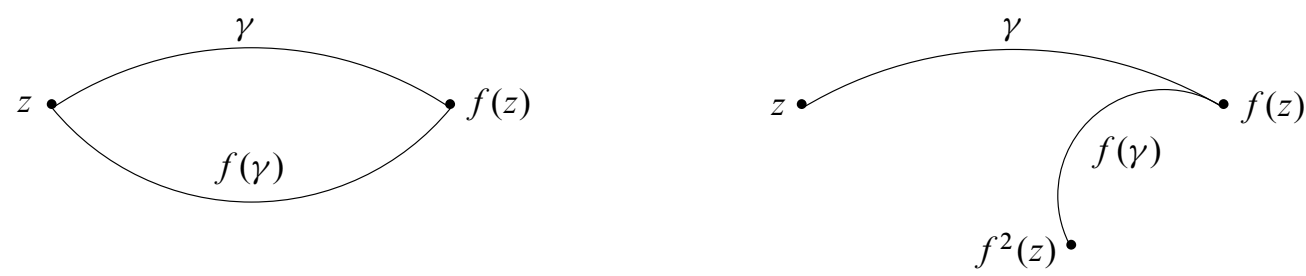

Figure 2

Il n'est pas difficile de montrer que tout point qui n'est pas fixe appartient à un arc de translation (nous en rappelerons d'ailleurs la preuve en Section 3). On en déduit l'énoncé suivant: 
Proposition 1.2 Soit $f \in \mathrm{Homeo}^{+}(\mathbf{D})$. S'il existe un point périodique qui n'est pas fixe, alors il existe un cercle d'indice 1 .

Démonstration Supposons que $z^{\prime}$ soit un point périodique de période $q \geq 2$ et considérons un arc de translation $\gamma$ joignant un point $z$ à $f(z)$ et contenant $z^{\prime}$. Remarquons maintenant que l'une des deux hypothèses de la Proposition 1.1 est nécessairement vérifiée.

Énonçons maintenant le lemme de Franks, introduit dans [7], qui étend le critère précédent. Pour cela, rappelons qu'une chaîne fermée de $f \in \mathrm{Homeo}^{+}(\mathbf{D})$ est une famille $\left(X_{i}\right)_{i \in \mathbb{Z} / r \mathbb{Z}}$ de parties de $\mathbf{D}$ telle que, pour tout $i \in \mathbb{Z} / r \mathbb{Z}, \bigcup_{k \geq 1} f^{k}\left(X_{i}\right) \cap$ $X_{i+1} \neq \varnothing$. L'entier $r$ est alors la longueur de la chaîne.

Proposition 1.3 Soit $f \in \mathrm{Homeo}^{+}(\mathbf{D})$. S'il existe une chaîne fermée de disques ouverts libres disjoints deux à deux, alors il existe un cercle d'indice 1 .

Démonstration Supposons qu'il existe une chaîne fermée de disques ouverts libres disjoints deux à deux. Parmi ces chaînes, choisissons en une de longueur minimale,

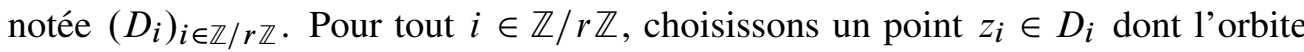
positive rencontre $D_{i+1}$, en notant $f^{k_{i}}\left(z_{i}\right)$ le premier point où cette orbite rencontre $D_{i+1}$. Puisque $r$ est minimal, on peut remarquer que tout point $f^{k}\left(z_{i}\right), 0<k<k_{i}$, est en dehors de chaque disque de la chaîne. On peut construire une isotopie $\left(h_{t}\right)_{t \in[0,1]}$ dans $\mathrm{Homeo}^{+}(\mathbf{D})$, issue de l'identité, telle que le support de chaque $h_{t}$ est inclus dans $\bigcup_{i \in \mathbb{Z} / r \mathbb{Z}} D_{i}$, et telle que $h_{1}$ envoie $f^{k_{i}}\left(z_{i}\right)$ sur $z_{i+1}$. Remarquons alors que $h_{1} \circ f$ a un point périodique de période $\sum_{i \in \mathbb{Z} / r \mathbb{Z}} k_{i} \geq 2$. D'après la Proposition 1.2, il existe un cercle $C \subset \mathbf{D} \backslash \operatorname{Fix}\left(h_{1} \circ f\right)$ tel que $i\left(h_{1} \circ f, C\right)=1$. Remarquons, puisque les disques $D_{i}$ sont libres, que Fix $\left(h_{t} \circ f\right)=\operatorname{Fix}(f)$, pour tout $t \in[0,1]$. On en déduit que la fonction $t \mapsto i\left(h_{t} \circ f, C\right)$ est bien définie. Elle est continue et à valeurs entières, elle est donc constante égale à 1 .

Nous dirons qu'un homéomorphisme $f \in \mathrm{Homeo}^{+}(\mathbf{D})$ est récurrent s'il vérifie les hypothèses du lemme de Franks, c'est-à-dire s'il existe une chaîne fermée de disques ouverts libres disjoints deux à deux. Nous pouvons alors énoncer deux classiques critères de récurrence:

Proposition 1.4 Un homéomorphisme $f \in \mathrm{Homeo}^{+}(\mathbf{D})$ est récurrent s'il vérifie l'une des conditions suivantes:

(i) il existe une chaîne fermée de parties libres, connexes par arcs et disjointes deux à deux; 
(ii) il existe un arc de translation $\gamma$ qui joint un point $z \notin \operatorname{Fix}\left(f^{2}\right)$ à $f(z)$ et un entier $k \geq 2$ tel que $f^{k}(\gamma) \cap \gamma \neq \varnothing$.

Démonstration Supposons que la condition (i) est vérifiée. Parmi les chaînes fermées de parties libres connexes par arcs et disjointes deux à deux, choisissons en une de

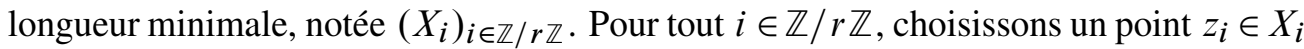
dont l'orbite positive rencontre $X_{i+1}$ et notons $f^{k_{i}}\left(z_{i}\right)$ le premier point où cette orbite rencontre $X_{i+1}$. D'après la minimalité de $r$, deux cas sont possibles:

- $r=1$ et $f^{k_{1}}\left(z_{1}\right)=z_{1}$;

- $f^{k_{i}}\left(z_{i}\right) \neq z_{i+1}$, pour tout $i \in \mathbb{Z} / r \mathbb{Z}$.

Dans le premier cas, $z_{1}$ est un point périodique de période $k_{1}>1$. Il suffit alors de choisir un disque ouvert contenant $z_{1}$ et libre pour obtenir une chaîne fermée de longueur 1: l'existence d'un point périodique qui n'est pas fixe implique la récurrence. Dans le second cas, puisque chaque $X_{i}$ est connexe par arcs, on peut trouver un segment $\gamma_{i} \subset X_{i}$ joignant $f^{k_{i}}\left(z_{i}\right)$ à $z_{i+1}$. Si on remplace chaque $\gamma_{i}$ par un disque ouvert $D_{i}$, voisinage assez proche de $\gamma_{i}$, on obtient notre chaîne.

Supposons maintenant que la condition (ii) est vérifiée. On peut toujours supposer que $f^{k^{\prime}}(\gamma) \cap \gamma=\varnothing$ si $2 \leq k^{\prime}<k$ et donc que $f^{k}(\gamma)$ ne contient pas $f(z)$. Deux cas sont alors possibles:

- $f^{k}(\gamma) \cap \gamma$ se réduit à un seul point $z^{\prime}$ et ce point est $f^{k+1}(z)$;

- il existe un point $z^{\prime \prime} \neq f(z)$ dont l'image par $f^{k}$ appartient à $\gamma$.

Plaçons nous dans le premier cas. Si $z^{\prime}=z$, alors $f$ a un point périodique de période $k+1 \geq 3$ et $f$ est donc récurrent. Si $z^{\prime} \neq z$, le sous-segment de $\gamma$ qui joint $z$ à $z^{\prime}$ est libre et définit une chaîne fermée de longueur 1, la condition (i) est vérifiée. Plaçons nous maintenant dans le second cas. Choisissons $z^{\prime} \neq f(z)$ sur $\gamma$ assez proche de $f(z)$ pour que le sous-segment de $\gamma$ qui joint $z^{\prime}$ à $f(z)$ ne contienne pas $z^{\prime \prime}$ et soit disjoint de $f^{k}(\gamma)$. Le sous-segment de $\gamma$ qui joint $z$ à $z^{\prime}$ est libre et définit une chaîne fermée de longueur 1 , la condition (i) est encore vérifiée.

Notre propriété de récurrence, qui est clairement invariante par conjugaison, est en fait très faible. Considérons, dans le plan complexe $\mathbb{C}$, l'homéomorphisme $g: z \mapsto-\frac{1}{2} z$. Le demi-plan de Poincaré $\Im(z)>0$ est un domaine périodique de $g$, de période 2 . Ainsi, tout homéomorphisme $f \in \mathrm{Homeo}^{+}(\mathbf{D})$ qui est conjugué à $g$ est récurrent. Un tel homéomorphisme a une dynamique Nord-Sud entre le bout de $\mathbf{D}$ et un point fixe.

Énonçons pour finir le critère suivant, dû à L Guillou et F Le Roux (voir [15, page 39]), qui est un peu plus délicat à montrer: 
Proposition 1.5 Soit $f \in \mathrm{Homeo}^{+}(\mathbf{D})$. Supposons qu'il existe une chaîne fermée $\left(X_{i}\right)_{i \in \mathbb{Z} / r \mathbb{Z}}$ de parties libres dont les intérieurs sont disjoints deux à deux et qui vérifient la propriété suivante: si $z$ et $z^{\prime}$ sont deux points distincts de $X_{i}$, il existe un segment joignant $z$ à $z^{\prime}$ tel que $\operatorname{int}(\gamma) \subset \operatorname{Int}\left(X_{i}\right)$. Alors $f$ est récurrent.

Pour démontrer le Théorème 0.1 , nous prouverons le résultat plus fort suivant:

Théorème 1.6 Si $f \in \mathrm{Homeo}^{+}$(D) vérifie les hypothèses (i), (ii), (iii), (iv) $d u$ Théorème 0.1 , alors $f$ est récurrent.

Le principe de la preuve est le suivant. Nous énoncerons un certain nombre d'hypothèses qui, si elles ne sont pas satisfaites par $f$, obligent $f$ à vérifier l'une des deux assertions (i) ou (ii) de la Proposition 1.4 (et donc à être récurrent). Dans le cas où toutes ces conditions sont satisfaites, nous montrerons que $f$ vérifie les hypothèses de la Proposition 1.5, et est donc également récurrent. Nous construirons pour cela une décomposition en briques libre particulière de $\mathbf{D} \backslash \operatorname{Fix}(f)$. Ces objets ont été introduits par M Flucher [6] puis développés par A Sauzet [17] (voir également [15] et [14]). Nous allons rappeler dans la Section 2 la définition ainsi que les principales propriétés des décompositions en briques.

\section{Décomposition en briques}

Une décomposition en briques d'une surface orientable $M$ (non nécessairement connexe) est la donnée d'un ensemble stratifié $\Sigma(\mathcal{D})$ de dimension un, appelé squelette de la décomposition $\mathcal{D}$, avec une sous-variété de dimension zéro $S$ tel que de tout sommet $s \in S$ sont issues (localement) exactement trois arêtes. Les briques sont les adhérences des composantes connexes de $M \backslash \Sigma(\mathcal{D})$ et les arêtes les adhérences des composantes connexes de $\Sigma(\mathcal{D}) \backslash S$. On notera $A$ l'ensemble des arêtes et $B$ l'ensemble des briques et on écrira $\mathcal{D}=(S, A, B)$ pour désigner une décomposition en briques. La Figure 3 donne un exemple de décomposition en briques.

Soit $\mathcal{D}=(S, A, B)$ une décomposition en briques de $M$. On dit qu'une partie $X$ de $B$ est connexe si, pour toutes briques $b, b^{\prime} \in X$, il existe une suite $\left(b_{i}\right)_{0 \leq i \leq n}$, où $b_{0}=b$ et $b_{n}=b^{\prime}$, telle que $b_{i}$ et $b_{i+1}$ sont adjacentes si $i \in\{0, \ldots, n-1\}$, c'est-à-dire contiennent une arête commune. On identifiera par la suite une partie $X$ de $B$ et la partie fermée de $M$, réunion des briques de $X$. Remarquons que $\partial X$ est une variété topologique de dimension 1 , remarquons également que la connexité de $X \subset B$ équivaut à la connexité de $X \subset M$ ainsi qu'à celle de $\operatorname{Int}(X) \subset M$. 


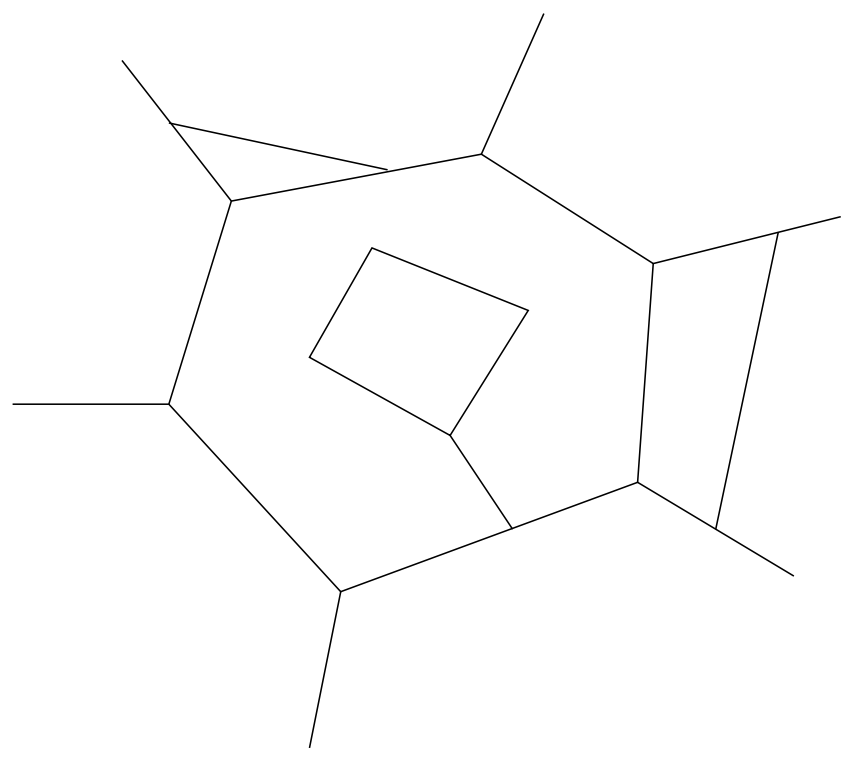

Figure 3

On dit que la décomposition $\mathcal{D}^{\prime}$ est une sous-décomposition de $\mathcal{D}$ si $\Sigma\left(\mathcal{D}^{\prime}\right)$ est inclus dans $\Sigma(\mathcal{D})$. Tout sommet de $\mathcal{D}^{\prime}$ est un sommet de $\mathcal{D}$, toute arête de $\mathcal{D}^{\prime}$ est réunion d'arêtes de $\mathcal{D}$ et toute brique de $\mathcal{D}^{\prime}$ réunion de briques de $\mathcal{D}$. Si $\left(X_{i}\right)_{i \in I}$ est une partition de $B$ en parties connexes, alors $\bigcup_{i \in I} \partial X_{i}$ est le squelette d'une sous-décomposition $\mathcal{D}^{\prime}$ de $\mathcal{D}$ dont les briques sont les $X_{i}$. En particulier si $\left(X_{i}\right)_{i \in I}$ est la partition en points, les briques de $\mathcal{D}^{\prime}$ et les briques de $\mathcal{D}$ sont les mêmes. Si $\mathcal{D}^{\prime}$ et $\mathcal{D}$ coïncident, on dira que $\mathcal{D}$ est une décomposition remplie: ceci signifie que toute arête de la décomposition est incluse dans exactement deux briques (ainsi la décomposition représentée sur la Figure 3 n'est pas remplie).

Si $f$ est un homéomorphisme de $M$, on définit naturellement une application $\varphi: \mathcal{P}(B) \rightarrow \mathcal{P}(B)$ en posant:

$$
\begin{aligned}
\varphi(X) & =\left\{b \in B \mid \text { il existe } b^{\prime} \in X \text { tel que } b \cap f\left(b^{\prime}\right) \neq \varnothing\right\} \\
& =\{b \in B \mid b \cap f(X) \neq \varnothing\} .
\end{aligned}
$$

Remarquons que $\varphi(X)$ est connexe si c'est le cas de $X$. Remarquons également que pour toute famille $\left(X_{i}\right)_{i \in I}$ de parties de $B$, on a:

$$
\varphi\left(\bigcup_{i \in I} X_{i}\right)=\bigcup_{i \in I} \varphi\left(X_{i}\right), \varphi\left(\bigcap_{i \in I} X_{i}\right) \subset \bigcap_{i \in I} \varphi\left(X_{i}\right) .
$$


On définit de façon analogue une application $\varphi_{-}: \mathcal{P}(B) \rightarrow \mathcal{P}(B)$ en posant:

$$
\begin{aligned}
\varphi_{-}(X) & =\left\{b \in B \mid \text { il existe } b^{\prime} \in X \text { tel que } b \cap f^{-1}\left(b^{\prime}\right) \neq \varnothing\right\} \\
& =\left\{b \in B \mid b \cap f^{-1}(X) \neq \varnothing\right\} .
\end{aligned}
$$

On définit le futur $b_{\geq}$et le passé $b_{\leq}$d'une brique $b$ en posant:

$$
b_{\geq}=\bigcup_{k \geq 0} \varphi^{k}(\{b\}), \quad b_{\leq}=\bigcup_{k \geq 0} \varphi_{-}^{k}(\{b\}) .
$$

On définit de même le futur strict $b_{>}$et le passé strict $b_{<}$en posant:

$$
b_{>}=\bigcup_{k \geq 1} \varphi^{k}(\{b\}), \quad b_{<}=\bigcup_{k \geq 1} \varphi_{-}^{k}(\{b\}) .
$$

Remarquons que les conditions suivantes sont équivalentes:

$$
b \in b_{>}, \quad b_{>}=b_{\geq}, \quad b \in b_{<}, \quad b_{<}=b_{\leq}, \quad b_{<} \cap b_{\geq} \neq \varnothing, \quad b_{\leq} \cap b_{>} \neq \varnothing .
$$

L'existence d'une brique $b \in B$ pour laquelle ces conditions sont vérifiées équivaut à l'existence d'une chaîne fermée constituée de briques.

On dira qu'une décomposition en briques $\mathcal{D}=(S, A, B)$ est libre si toute brique de $\mathcal{D}$ est libre. Si $f$ n'a pas de point fixe, il est toujours possible, quitte à choisir les briques assez petites, de construire une décomposition en briques libre. On déduit immédiatement de la Proposition 1.5:

Proposition 2.1 Soit $f \in \mathrm{Homeo}^{+}(\mathbf{D})$ un homéomorphisme différent de l'identité et $\mathcal{D}=(S, A, B)$ une décomposition en briques libre de $\mathbf{D} \backslash \operatorname{Fix}(f)$. S'il existe $b \in B$ tel que $b \in b_{>}$, alors $f$ est récurrent.

Rappelons la définition de décomposition libre maximale, notion introduite par Sauzet dans sa thèse [17]. Soit $f$ un homéomorphisme sans point fixe d'une surface $M$ et $\mathcal{D}$ une décomposition en briques libre. On peut trouver une sous-décomposition $\mathcal{D}^{\prime}$ de $\mathcal{D}$ qui est libre et telle que toute sous-décomposition stricte de $\mathcal{D}^{\prime}$ n'est plus libre. On dit que $\mathcal{D}^{\prime}$ est une décomposition en briques libre maximale. Remarquons que toute décomposition en briques libre maximale est remplie. Remarquons également que la réunion de deux briques adjacentes n'est pas libre. Dans sa thèse, Sauzet montre, dans le cas où $M=\mathbf{D}$, que toute brique $b$ est adjacente à une brique qui rencontre $f(b)$ et à une brique qui rencontre $f^{-1}(b)$. Nous allons prouver un résultat similaire si $M$ est le complémentaire de l'ensemble des points fixes d'un homéomorphisme non trivial $f \in \mathrm{Homeo}^{+}(\mathbf{D})$. Plus précisément: 
Proposition 2.2 Soit $f \in \mathrm{Homeo}^{+}(\mathbf{D})$ un homéomorphisme différent de l'identité et $\mathcal{D}=(S, A, B)$ une décomposition en briques libre maximale de $\mathbf{D} \backslash$ Fix $(f)$. Alors, l'une au moins des assertions suivantes est vraie:

(i) il existe une chaîne fermée de briques de $B$ (et $f$ est donc récurrent);

(ii) toute brique $b$ est adjacente à une brique qui rencontre $f(b)$ et à une brique qui rencontre $f^{-1}(b)$.

Dans le second cas, on en déduit que les parties $b_{\geq}, b_{>}, b_{\leq}$et $b_{<}$sont connexes.

Démonstration Expliquons d'abord le dernier point. Si (ii) est vérifié, les ensembles $\{b\} \cup \varphi\left(\{b\}\right.$ et $\{b\} \cup \varphi_{-}(\{b\})$ sont connexes et on peut écrire

$$
b_{\geq}=\bigcup_{k \geq 0} \varphi^{k}(\{b\})=\bigcup_{k \geq 0} \varphi^{k}(\{b\} \cup \varphi(\{b\})) .
$$

Ainsi, $b \geq$ est connexe, étant l'union de parties connexes $X_{k}=\varphi^{k}(\{b\} \cup \varphi(\{b\}))$ telles que $X_{k} \cap X_{k+1} \neq \varnothing$. De même on peut écrire

$$
b_{>}=\bigcup_{k \geq 1} \varphi^{k}(\{b\} \cup \varphi(\{b\}))
$$

ainsi que

$$
b_{\leq}=\bigcup_{k \geq 0} \varphi_{-}^{k}\left(\{b\} \cup \varphi_{-}(\{b\})\right), \quad b_{<}=\bigcup_{k \geq 1} \varphi_{-}^{k}\left(\{b\} \cup \varphi_{-}(\{b\})\right) .
$$

Prouvons maintenant que l'une des assertions (i) ou (ii) est vraie. Commençons par compactifier $\mathbf{D}$ en ajoutant le bout $\infty$ et prolongeons $f$ en un homéomorphisme de la sphère $\mathbf{D} \sqcup\{\infty\}$ qui fixe $\infty$. On sait, d'après M. Brown et J. Kister [3], que toute composante connexe de $\mathbf{D} \backslash \operatorname{Fix}(f)$ est fixe (et n'est donc pas libre). Ainsi la frontière de toute brique $b$ est non vide. C'est une sous-variété topologique de dimension un, et donc une réunion de cercles et de droites de $\mathbf{D} \backslash \operatorname{Fix}(f)$.

Commençons par le cas où $\partial b$ admet au moins deux composantes connexes $\Gamma_{1}$ et $\Gamma_{2}$. Fixons une arête $a_{1} \subset \Gamma_{1}$ (resp. $a_{2} \subset \Gamma_{2}$ ) et notons $b_{1}$ (resp. $b_{2}$ ) la brique adjacente à $b$ qui contient $a_{1}$ (resp. $a_{2}$ ). On sait que ni $b \cup b_{1}$, ni $b \cup b_{2}$ n'est libre, puisque $\mathcal{D}$ est une décomposition libre maximale. Ainsi, $b_{1}$ et $b_{2}$ rencontrent $f(b) \cup f^{-1}(b)$. Si $b$ ne vérifie pas la condition (ii), l'un des ensembles $f(b)$ ou $f^{-1}(b)$ rencontre à la fois $b_{1}$ et $b_{2}$. On peut supposer, sans perte de généralité, que c'est $f(b)$. L'ensemble connexe $\varphi(\{b\})$ contient donc $b_{1}$ et $b_{2}$. Nous pouvons donc construire deux segments $\gamma$ et $\gamma^{\prime}$ joignant un point $z_{1} \in \operatorname{int}\left(a_{1}\right)$ à un point $z_{2} \in \operatorname{int}\left(a_{2}\right)$, le premier prenant ses valeurs, sauf aux extrémités, dans l'intérieur de $\varphi(\{b\})$, le second prenant ses valeurs, 
sauf aux extrémités, dans l'intérieur de $b$. Le réunion des deux segments est un cercle $C \subset b_{\geq}$qui rencontre transversalement chaque $\operatorname{arc} \Gamma_{1}$ et $\Gamma_{2}$ en un unique point. Les $\operatorname{arcs} \Gamma_{1}$ et $\Gamma_{2}$ ne peuvent donc pas être des cercles, ce sont des droites de $\mathbf{D} \backslash \operatorname{Fix}(f)$. On peut alors trouver deux points d'accumulation (dans $\mathbf{D} \sqcup\{\infty\}$ ) de $\Gamma_{1}$ qui sont séparés par $C$. Il s'agit bien sûr de points fixes de $f$ et ils appartiennent donc également à l'adhérence (dans $\mathbf{D} \sqcup\{\infty\}$ ) de $f^{-1}(b)$. On en déduit que $f^{-1}(b)$ rencontre $C$ et donc que $b_{<} \cap b_{\geq} \neq \varnothing$. Ainsi $f$ vérifie la Proposition 1.5 et est donc récurrent. Remarquons que nous venons également de montrer que $f$ est récurrent si $\partial b$ a au moins trois composantes connexes, puisque le raisonnement précédent s'applique à au moins un couple de composantes connexes de $\partial b$.

Supposons maintenant que $\partial b$ a une seule composante connexe $\Gamma$ et que c'est un cercle. Supposons, là encore, que $b$ ne vérifie pas la condition (ii) et montrons qu'il existe une chaîne fermée de briques de $B$. Nous sommes dans une situation déjà étudiée par Sauzet. Il y a un nombre fini de briques adjacentes à $b$ et on peut supposer que l'image par $f$ de chacune de ces briques rencontre $b$. Si $b^{\prime}$ est adjacente à $b$, l'image par $f$ de l'arête commune à $b$ et $b^{\prime}$ est disjointe de $b$ puisque $b$ est libre. Ainsi $f\left(b^{\prime}\right)$ rencontre nécessairement une autre brique adjacente à $b$. On en déduit immédiatement l'existence d'une chaîne fermée de briques adjacentes à $b$.

Il reste à étudier le cas le plus difficile, où $\partial b$ a une seule composante connexe $\Gamma$ qui est une droite de $\mathbf{D} \backslash$ Fix $(f)$. Nous sommes dans une situation également étudiée par Sauzet (voir [17] ou [14]). Nous allons supposer que toute brique adjacente à $b$ rencontre $f^{-1}(b)$ et en déduire que $f$ est récurrent. Nous notons $W$ la composante connexe de $\mathbf{D} \backslash \operatorname{Fix}(f)$ qui contient $b$. Nous savons d'après [3] qu'elle est invariante par $f$ et qu'elle contient également $f(b)$ et $f^{-1}(b)$. On peut bien sûr supposer que $f(b)$ et $f^{-1}(b)$ sont disjoints, puisque dans le cas contraire, $f$ est récurrent. Soit $b_{0}$ une brique adjacente à $b$. On sait que $f\left(b_{0}\right)$ rencontre à la fois $b$ et $f(b)$. On peut donc trouver un segment $\gamma_{0}$ joignant un point de $b$ à un point de $f(b)$ et contenu dans $f\left(b_{0}\right)$. Quitte à restreindre ce segment, on peut supposer qu'il joint un point $z_{0} \in \partial b$ à un point $z_{0}^{\prime} \in \partial f(b)$ et que $\operatorname{int}\left(\gamma_{0}\right) \subset W \backslash(b \cup f(b))$. On peut écrire $\Gamma=\Gamma_{0}^{-} \Gamma_{0}^{+}$comme assemblage de deux demi-droites ayant $z_{0}$ comme extrémité commune. Fixons alors $z_{0}^{-} \in \operatorname{int}\left(\Gamma_{0}^{-}\right)$et $z_{0}^{+} \in \operatorname{int}\left(\Gamma_{0}^{+}\right)$et remarquons que tout point de $W \backslash(b \cup f(b))$, proche de $z_{0}^{-}$, est séparé dans $W \backslash(b \cup f(b)) \operatorname{par} \operatorname{int}\left(\gamma_{0}\right)$ de tout point de $W \backslash(b \cup f(b))$ proche de $z_{0}^{+}$. En effet, dans le cas contraire, on pourrait construire un cercle $C \subset W \backslash(b \cup f(b))$ qui intersecte $\gamma_{0}$ en un unique point et ceci de façon transverse. Le cercle $C$ séparerait donc $b$ et $f(b)$ dans $\mathbf{D}$. Notons $\bar{b}$ et $\overline{f(b)}$ les adhérences de $b$ et $f(b)$ dans D. La courbe $C$ séparerait $\bar{b}$ et $\overline{f(b)}$ dans $\mathbf{D}$ puisque $b$ et $f(b)$ sont fermés dans $W$. Notons maintenant que $\bar{b}$ contient un point fixe (il suffit de choisir un point d'accumulation de $\Gamma$ ). Ce point 
fixe appartient également à $f \overline{(b)}$. Ceci contredit la propriété de séparation. Dans le cas où $f^{-1}(b) \cap \gamma_{0} \neq \varnothing$, l'homéomorphisme $f$ est récurrent. En effet, $\gamma_{0}$ est libre, puisqu'il est contenu dans $f\left(b_{0}\right)$. On peut donc épaissir $\gamma_{0}$ pour construire un disque libre $D_{0} \subset W$ dont l'intérieur est disjoint de $b$ et $f(b)$ et ce disque vérifie $f\left(D_{0}\right) \cap b=\varnothing$ si $f^{-1}(b) \cap \gamma_{0} \neq \varnothing$. Puisque $f(b) \cap D_{0} \neq \varnothing$, on peut appliquer la Proposition 1.5 pour conclure que $f$ est récurrent. Nous supposerons donc que $f^{-1}(b) \cap \gamma_{0}=\varnothing$. Puisque $f^{-1}(b)$ est connexe, on peut toujours supposer, quitte à intervertir $\Gamma_{0}^{-}$et $\Gamma_{0}^{+}$, qu'un point dans $W \backslash(b \cup f(b))$ qui est proche de $z_{0}^{-}$est séparé de $f^{-1}(b)$ dans $W \backslash(b \cup f(b))$ par $\operatorname{int}\left(\gamma_{0}\right)$. Par hypothèse, toute brique $b^{\prime}$ adjacente à $b$ intersecte $f^{-1}(b)$, elle rencontre donc $\gamma_{0}$ si elle rencontre $\Gamma_{0}^{-}$. Puisque $\gamma_{0}$ est une partie compacte de $W$, il n'y a donc qu'un nombre fini de briques adjacentes à $b$ qui rencontrent $\Gamma_{0}^{-}$. Il existe donc une brique $b_{1}$, adjacente à $b$, qui rencontre $\Gamma_{0}^{-}$en des points arbitrairement éloignés de $z_{0}$. On construit alors de façon similaire un segment $\gamma_{1} \subset f\left(b_{1}\right)$ joignant un point $z_{1} \in \partial b$ à un point $z_{1}^{\prime} \in \partial f(b)$ tel que $\operatorname{int}\left(\gamma_{1}\right) \subset W \backslash(b \cup f(b))$. On écrit alors $\Gamma=\Gamma_{1}^{-} \Gamma_{1}^{+}$comme assemblage de deux demi-droites ayant $z_{1}$ comme extrémité commune en faisant le choix suivant: $\Gamma_{1}^{-} \cap \Gamma_{0}^{-}$ est une demi-droite. On fixe alors $z_{1}^{-} \in \operatorname{int}\left(\Gamma_{1}^{-}\right)$et $z_{1}^{+} \in \operatorname{int}\left(\Gamma_{1}^{+}\right)$. Si $f^{-1}(b)$ rencontre $\gamma_{1}$, alors $f$ est récurrent. Sinon, la partie connexe $b_{1} \cup f^{-1}(b)$ est disjointe de $\gamma_{1}$ et rencontre $\Gamma_{1}^{-}$. On en déduit que tout point de $W \backslash b \cup f(b)$ qui est proche de $z_{1}^{+}$est séparé de $f^{-1}(b)$ dans $W \backslash(b \cup f(b))$ par int $\left(\gamma_{1}\right)$. On sait donc qu'il n'y a qu'un nombre fini de briques adjacentes à $b$ qui rencontrent $\Gamma_{1}^{+}$. On vient de prouver qu'il n'y a qu'un nombre fini de briques adjacentes à $b$. On remarque alors que le raisonnement fait dans le cas où $\Gamma$ est un cercle est encore valable.

Nous allons conclure cette section en donnant le plan de la démonstration du Théorème 0.1. Pour prouver ce théorème, la question qui vient naturellement à l'esprit est la suivante:

Si $f \in$ Homeo $^{+}$(D) vérifie les hypothèses (i), (ii), (iii), (iv) du Théorème 0.1 , et si $\mathcal{D}$ est une décomposition en briques libre maximale de $\mathbf{D} \backslash \operatorname{Fix}(f)$, existe-t-il une chaîne fermée constituée de briques de la décomposition?

Nous ne savons pas répondre à cette question. Nous verrons, par contre, qu'il est possible de construire une décomposition en briques libre maximale $\mathcal{D}$ de $\mathbf{D} \backslash \operatorname{Fix}(f)$, pour laquelle la réponse est oui. Pour obtenir cette chaîne, nous nous servirons de la connexité du futur, du passé, du futur strict et du passé strict de chaque brique. Il faudra cependant rendre utilisables, dans l'étude de la décomposition, les hypothèses dynamiques et combinatoires du Théorème 0.1 . Ces quatre hypothèses portent sur 
des ensembles discrets, les orbites des $z_{i}$. La première étape est de construire des ensembles unidimensionnels sur lesquels, on peut traduire ces hypothèses.

Nous commencerons dans la Section 3 par construire pour tout $i \in \mathbb{Z} / n \mathbb{Z}$ et tout $k \in \mathbb{Z}$ un arc de translation $\gamma_{i}^{k}$ de $f^{k}\left(z_{i}\right)$, paramétré par $t \in[k, k+1]$, de telle façon que l'arc $\Gamma_{i}=\prod_{k \in \mathbb{Z}} \gamma_{i}^{k}$ paramétré par $t \in \mathbb{R}$ vérifie:

- $\lim _{t \rightarrow-\infty} \Gamma_{i}(t)=\alpha_{i}$

- $\lim _{t \rightarrow+\infty} \Gamma_{i}(t)=\omega_{i}$

- pour tous réels $t$ et $t^{\prime}$, on a $f\left(\Gamma_{i}(t)\right) \neq \Gamma_{i}\left(t^{\prime}\right)$ si $t^{\prime} \leq t$.

L'arc $\Gamma_{i}$ contient l'orbite $O_{i}$ du point $z_{i}$. Si chaque $\gamma_{i}^{k}$ est un arc simple, il n'en est pas de même de chaque $\Gamma_{i}$. Dans la Section 4, nous verrons que l'on peut modifier les segments $\gamma_{i}^{k}$ pour avoir une situation "générique" au sens suivant:

- tout point $f^{k}\left(z_{i}\right)$ est un point simple de $\Gamma_{i}$;

- les arcs $\Gamma_{i}$ n'ont pas de points triples;

- $\Gamma_{i} \cap O_{i^{\prime}}=\varnothing$, si $i \neq i^{\prime}$;

- $\Gamma_{i} \cap \Gamma_{i^{\prime}}$ est fini, si $i \neq i^{\prime}$;

- $\Gamma_{i} \cap \Gamma_{i^{\prime}}$ ne contient aucun point double ni de $\Gamma_{i}$, ni de $\Gamma_{i^{\prime}}$, si $i \neq i^{\prime}$;

- $\Gamma_{i} \cap \Gamma_{i^{\prime}} \cap \Gamma_{i^{\prime \prime}}=\varnothing$ si $i$, $i^{\prime}$ et $i^{\prime \prime}$ sont distincts.

En utilisant les hypothèses combinatoires (ii) et (iii) du Théorème 0.1 , nous démontrerons dans la Section 5 l'existence d'une configuration de connexité critique $\left(t_{i}\right)_{i \in \mathbb{Z} / n \mathbb{Z}}$ au sens suivant:

(A) les ensembles $\Gamma_{i}(]-\infty, t_{i}[), i \in \mathbb{Z} / n \mathbb{Z}$, sont disjoints deux à deux;

(B) pour tout $i \in \mathbb{Z} / n \mathbb{Z}$, il existe $i^{\prime} \notin\{i-1, i\}$ tel que

$$
\left.\left.\left.\left.\Gamma_{i}(]-\infty, t_{i}\right]\right) \cap \Gamma_{i^{\prime}}(]-\infty, t_{i^{\prime}}\right]\right) \neq \varnothing .
$$

Il est facile de voir qu'il existe un entier $K>0$ tel que si $i$ et $i^{\prime}$ sont distincts, alors

$$
\left.\left.\Gamma_{i}(]-\infty,-K\right]\right) \cap \Gamma_{i^{\prime}}=\Gamma_{i}\left(\left[K,+\infty[) \cap \Gamma_{i^{\prime}}=\varnothing .\right.\right.
$$

On en déduit que $\left|t_{i}\right|<K$ pour tout $i \in \mathbb{Z} / n \mathbb{Z}$. La propriété (B) implique alors que l'ensemble $\left.\left.\Gamma_{i-1}(]-\infty,-K\right]\right)$ est séparé dans $\mathbf{D}$ de $\Gamma_{i-1}\left(\left[K,+\infty[)\right.\right.$ par $\left.\left.\Gamma_{i}(]-\infty, t_{i}\right]\right) \cup$ $\left.\left.\Gamma_{i^{\prime}}(]-\infty, t_{i^{\prime}}\right]\right)$. Nous verrons que l'on peut de plus supposer que pour tout $i \in \mathbb{Z} / n \mathbb{Z}$, il existe $i^{\prime} \neq i$ tel que $\Gamma_{i}\left(t_{i}\right) \in \Gamma_{i^{\prime}}(]-\infty, t_{i^{\prime}}[)$.

Dans la Section 6, nous construirons à partir des ensembles $\left.\left.\Gamma_{i}(]-\infty, t_{i}\right]\right)$ une famille d'arbres $\left(A_{i}^{-}\right)_{i \in \mathbb{Z} / n \mathbb{Z}}$, où $\left.\left.A_{i}^{-} \subset \Gamma_{i}(]-\infty, t_{i}\right]\right)$ sera obtenu en enlevant des boucles de $\left.\left.\Gamma_{i}(]-\infty, t_{i}\right]\right)$. L'arbre $A_{i}^{-}$contiendra tous les points $f^{k}\left(z_{i}\right), k \leq t_{i}$, l'extrémité $\Gamma_{i}\left(t_{i}\right)$ ainsi que toutes les extrémités $\Gamma_{i^{\prime}}\left(t_{i^{\prime}}\right)$ qui sont sur $\left.\left.\Gamma_{i}(]-\infty, t_{i}\right]\right)$. Ainsi: 
$\left(\mathbf{A}^{\prime}\right)$ les ensembles $A_{i}^{-} \backslash\left\{\Gamma_{i}\left(t_{i}\right)\right\}$ sont disjoints deux à deux;

$\left(\mathbf{B}^{\prime}\right)$ pour tout $i \in \mathbb{Z} / n \mathbb{Z}$, il existe $i^{\prime} \notin\{i-1, i\}$ tel que $A_{i}^{-} \cap A_{i^{\prime}}^{-} \neq \varnothing$.

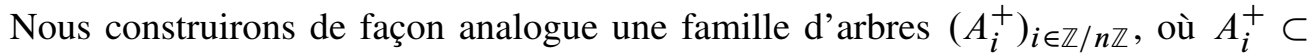
$\Gamma_{i}\left(\left[K,+\infty[)\right.\right.$ contiendra tous les points $f^{k}\left(z_{i}\right), k>K$. Chaque arbre $A_{i}^{+}$sera disjoint de tous les arbres $A_{i^{\prime}}^{-}$, ainsi que de tous les $A_{i^{\prime}}^{+}, i^{\prime} \neq i$. La propriété $\left(\mathbf{B}^{\prime}\right)$ implique qu'il existe une composante connexe de $A_{i}^{-} \cup A_{i^{\prime}}^{-}$qui contient tous les points $f^{k}\left(z_{i-1}\right), k \leq-K$, et une autre composante connexe qui contient l'arbre $A_{i-1}^{+}$et donc tous les points $f^{k}\left(z_{i-1}\right), k>K$.

Le caractère "presque disjoint" de tous ces arbres, illustré par la propriété $\left(\mathbf{A}^{\prime}\right)$, va nous permettre de construire dans la Section 7 une décomposition en briques libre maximale modelée sur ces arbres. Cette construction se fera en plusieurs étapes, la première étape consistant à épaissir les arbres, ce qui sera possible justement grâce à la propriété $\left(\mathbf{A}^{\prime}\right)$. La propriété essentielle vérifiée par la décomposition finale $\mathcal{D}^{\prime \prime}=\left(S^{\prime \prime}, A^{\prime \prime}, B^{\prime \prime}\right)$ sera la suivante:

Pour tout $i \in \mathbb{Z} / n \mathbb{Z}$, il existe une famille de briques $\left(b^{\prime \prime l}{ }_{i}\right)_{l \neq 0}$ telle que:

- $A_{i}^{-} \subset \bigcup_{l<0} b_{i}^{\prime \prime l}$;

- $A_{i}^{+} \subset \bigcup_{l>0} b_{i}^{\prime \prime l}$;

- $\Gamma_{i}\left(t_{i}\right) \in b_{i}^{\prime \prime-1}$;

- $b_{i}^{\prime \prime \prime} l_{i}^{\prime} \in\left(b_{i}^{\prime \prime l}\right)_{i}$ si $0<l<l^{\prime}$, si $l<l^{\prime}=-1$ ou si $l<0<l^{\prime}$.

Grâce à la propriété $\left(\mathbf{B}^{\prime}\right)$, ou plutôt à la propriété de séparation qu'elle implique, on montrera dans la Section 8 que le futur $\left(b_{i-1}^{\prime \prime-1}\right)_{\geq}$de $b_{i-1}^{\prime \prime}-1$, qui peut être supposé connexe d'après la Proposition 2.2, et qui contient $b_{i-1}^{\prime \prime-1}$, doit contenir une brique $b^{\prime \prime}{ }_{i}$, $l<0$, ou une brique $b^{\prime \prime l^{\prime}}, l^{\prime}<0$. Ainsi $\left(b_{i-1}^{\prime \prime-1}\right) \geq$ contient $b_{i}^{\prime \prime-1}$ ou $b_{i^{\prime}}^{\prime \prime-1}$. Dans le cas où les briques $b^{\prime \prime}-1, i \in \mathbb{Z} / n \mathbb{Z}$, sont toutes distinctes, on en déduit immédiatement qu'il existe une chaîne fermée de briques. Il restera à étudier le cas où deux au moins de ces briques sont égales. Un argument de connexité du même type permettra de prouver que cette brique $b^{\prime \prime}$ vérifie $b^{\prime \prime} \in b_{>}^{\prime \prime}$.

Il faut noter que les propositions que nous verrons dans la suite de l'article sont du même type que la Proposition 2.2. En d'autres termes, si dans la construction précédente, on ne peut pas passer d'une étape à l'étape suivante, c'est que $f$ est récurrent. 


\section{Construction des $\operatorname{arcs} \Gamma_{i}$}

Nous fixons jusqu'à la fin de l'article un homéomorphisme $f \in \mathrm{Homeo}^{+}$(D) qui vérifie les hypothèses du Théorème 0.1 . Nous notons alors $O_{i}$ l'orbite de $z_{i}$ et posons $z_{i}^{k}=f^{k}\left(z_{i}\right)$. Nous prouverons dans cette section le résultat suivant:

Proposition 3.1 L'une au moins des assertions suivantes est vraie.

(i) L'homéomorphisme $f$ est récurrent.

(ii) Pour tout $i \in \mathbb{Z} / n \mathbb{Z}$, on peut construire une suite $\left(\gamma_{i}^{k}\right)_{k \in \mathbb{Z}}$ de segments tels que:

- chaque $\gamma_{i}^{k}$ est un arc de translation qui joint $z_{i}^{k}$ à $z_{i}^{k+1}$;

- $f\left(\gamma_{i}^{k}\right) \cap \gamma_{i}^{k^{\prime}}=\varnothing$ si $k^{\prime}<k$;

- la suite $\left(\gamma_{i}^{k}\right)_{k \leq 0}$ converge vers $\left\{\alpha_{i}\right\}$ pour la distance de Hausdorff;

- la suite $\left(\gamma_{i}^{k}\right)_{k \geq 0}$ converge vers $\left\{\omega_{i}\right\}$.

Pour simplifier les notations, nous omettrons dans cette section l'indice $i$. Commençons par un lemme.

Lemme 3.2 On peut construire une suite $\left(\gamma^{\prime k}\right)_{k \in \mathbb{Z}}$ de segments disjoints deux à deux tels que:

- $z^{k} \in \gamma^{\prime k}$

- $\gamma^{\prime k} \cap \operatorname{Fix}(f)=\varnothing$;

- $f\left(\gamma^{\prime k}\right) \cap \gamma^{\prime k} \neq \varnothing$;

- la suite $\left(\gamma^{\prime k}\right)_{k \leq 0}$ converge vers $\{\alpha\}$;

- la suite $\left(\gamma^{\prime k}\right)_{k \geq 0}$ converge vers $\{\omega\}$.

Démonstration On construit aisément un homéomorphisme $h: \mathbf{D} \rightarrow]-1,1\left[{ }^{2}\right.$ tel que

$$
\begin{array}{r}
\lim p_{1}\left(z^{\prime}\right)=-1 \Leftrightarrow \lim h^{-1}\left(z^{\prime}\right)=\alpha, \\
\lim p_{1}\left(z^{\prime}\right)=1 \Leftrightarrow \lim h^{-1}\left(z^{\prime}\right)=\omega,
\end{array}
$$

où $p_{1}, p_{2}$ sont les deux projections définies sur $] 0,1\left[{ }^{2}\right.$. Par un simple argument de récurrence, utilisant en particulier le fait que $\lim _{k \rightarrow-\infty} z^{k}=\alpha$ et $\lim _{k \rightarrow+\infty} z^{k}=\omega$, on peut toujours modifier $h$ pour que $p_{1}$ soit injective sur $h(O)$, ce qu'on supposera dorénavant. Puisque $p_{1}$ est injective sur $h(O)$, puisque $\lim _{k \rightarrow-\infty} p_{1}\left(h\left(z^{k}\right)\right)=-1$ et puisque $\lim _{k \rightarrow+\infty} p_{1}\left(h\left(z^{k}\right)\right)=1$, on peut, quitte à composer $h$ par un homéomorphisme de $]-1,1\left[^{2}\right.$ fixant chaque verticale, choisir la suite $p_{2}\left(h\left(z^{k}\right)\right)_{k \in \mathbb{Z}}$ arbitraire. 
On supposera donc que cette suite est strictement croissante. Pour simplifier l'écriture, on omettra $h$ dans la suite; on supposera en fait $p_{1}$ et $p_{2}$ définies sur $\mathbf{D}$, via la carte globale $h$.

Notons $I_{k}$ l'intervalle ouvert de ]-1,1[ délimité par $p_{1}\left(z^{k}\right)$ et $p_{1}\left(z^{k+1}\right)$ et posons $\left.U^{k}=I_{k} \times\right] p_{2}\left(z^{k}\right), p_{2}\left(z^{k+1}\right)\left[\right.$. Remarquons que les suites $\left(\bar{U}^{k}\right)_{k \leq 0}$ et $\left(\bar{U}^{k}\right)_{k \geq 0}$ sont formées de disques fermés de $\mathbf{D}$ et convergent respectivement vers $\{\alpha\}$ et $\{\omega\}$.

L'ensemble des points de $\mathbf{D}$ dont l'image par $f$ est sur la même verticale qu'un élément de $O$ est un $F_{\sigma}$ d'intérieur vide, on peut donc choisir un point $z^{\prime 0} \in U^{0}$ en dehors de cet ensemble. Pour les mêmes raisons, on peut choisir $z^{\prime 1} \in U^{1}$ en dehors de cet ensemble, qui de plus n'appartient pas à la verticale passant par $z^{\prime 0}$, ni à l'image par $f$ de cette verticale, ni à son image inverse. En réitérant cet argument, on peut construire par un simple procédé de récurrence, une suite $\left(z^{\prime k}\right)_{k \in \mathbb{Z}}$ telle que

- $z^{\prime k} \in U^{k}$

- $p_{1}\left(f\left(z^{\prime k}\right)\right) \neq p_{1}\left(z^{k^{\prime}}\right)$ pour tous entiers $k, k^{\prime}$;

- $p_{1}\left(z^{\prime k}\right) \neq p_{1}\left(z^{\prime k^{\prime}}\right)$ si $k \neq k^{\prime}$;

- $p_{1}\left(f\left(z^{\prime k}\right)\right) \neq p_{1}\left(z^{\prime k^{\prime}}\right)$ si $k \neq k^{\prime}$.

Puisque, par hypothèse, $f$ se prolonge en un homéomorphisme de $\mathbf{D} \cup\{\alpha, \omega\}$, et puisque les suites $\left(z^{\prime k}\right)_{k \geq 0}$ et $\left(z^{\prime k}\right)_{k \leq 0}$ convergent respectivement vers $\omega$ et $\alpha$, nous en déduisons que les suites $\left(f\left(z^{\prime k}\right)\right)_{k \geq 0}$ et $\left(f\left(z^{\prime k}\right)\right)_{k \geq 0}$ convergent également respectivement vers $\omega$ et $\alpha$. Quitte à composer $h$ par un homéomorphisme de $]-1,1\left[{ }^{2}\right.$ fixant chaque verticale, on peut donc supposer que $p_{2}\left(z^{k}\right)<p_{2}\left(f\left(z^{\prime k}\right)\right)<p_{2}\left(z^{k+1}\right)$. On peut alors construire une suite $\left(I^{\prime k}\right)_{k \in \mathbb{Z}}$ d'intervalles ouverts relativement compacts de ]-1, 1[, telle que:

- $I^{\prime k}$ contient $I^{k}$;

- $\left.U^{\prime k}=I^{\prime k} \times\right] p_{2}\left(z^{k}\right), p_{2}\left(z^{k+1}\right)\left[\right.$ contient $z^{\prime k}$ et $f\left(z^{\prime k}\right)$;

- les suites $\left(\bar{U}^{\prime k}\right)_{k \leq 0}$ et $\left(\bar{U}^{\prime k}\right)_{k \geq 0}$ de disques fermés de $\mathbf{D}$, convergent respectivement vers $\{\alpha\}$ et $\{\omega\}$.

Chaque segment $\gamma^{\prime k}$ que nous allons construire sera contenu dans $U_{k}^{\prime} \cup\left\{z^{k}\right\}$. Ces segments seront donc disjoints et vérifieront les deux conditions de convergence demandées. Il reste à construire les $\gamma^{\prime k}$ pour qu'ils vérifient les trois autres conditions.

Supposons d'abord qu'il n'y ait qu'un nombre fini de points fixes dans $U^{\prime k}$. Nous pouvons alors supposer que $z^{\prime k}$ n'est pas fixe et trouver un segment issu de $z^{k}$, à valeurs dans $U^{\prime k} \cup\left\{z^{k}\right\}$, disjoint de $\operatorname{Fix}(f)$ et contenant à la fois $z^{\prime k}$ et $f\left(z^{\prime k}\right)$. Ce segment convient. 
Supposons maintenant qu'il y ait une infinité de points fixes dans $U^{\prime k}$. On peut construire dans $U^{\prime k} \cup\left\{z^{k}\right\}$ trois segments issus de $z^{k}$, n'ayant que ce point en commun, et aboutissant chacun en un point fixe. Quitte à restreindre chaque segment, on peut supposer que l'extrémité finale est l'unique point fixe rencontré. Dans le cas où l'un des segments rencontre son image par $f$ en un point qui n'est pas l'extrémité fixe, on peut trouver un sous-segment d'extrémité $z^{k}$, qui ne rencontre pas Fix $(f)$ et qui n'est pas libre. Ce sous-segment $\gamma^{\prime k}$ convient. Supposons maintenant que chacun des trois segments ne rencontre son image qu'en son extrémité fixe. Remarquons que si l'image par $f$ de chacun des trois segments était disjointe des deux autres segments, alors $f$ devrait renverser l'orientation (voir Figure 4).

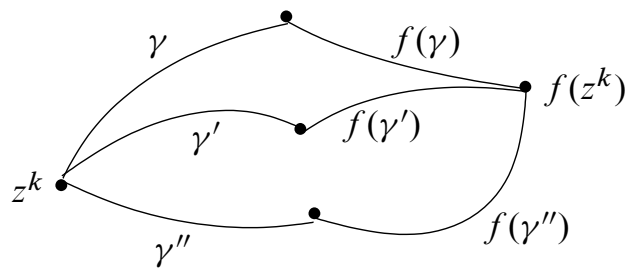

Figure 4

Puisque $f$ préserve l'orientation, on peut donc trouver deux segments, parmi les trois, dont la réunion n'est pas libre. Enlevons à chacun de nos deux segments un petit voisinage de l'extrémité fixe, la réunion des deux segments diminués est un segment $\gamma^{\prime k}$ qui convient.

On en déduit alors:

Lemme 3.3 On peut construire une suite $\left(D^{\prime k}\right)_{k \in \mathbb{Z}}$ de disques fermés disjoints deux à deux tels que:

- $z^{k} \in \partial D^{\prime k}$

- $D^{\prime k} \cap \operatorname{Fix}(f)=\varnothing$;

- $f\left(D^{\prime k}\right) \cap D^{\prime k} \neq \varnothing$;

- $f\left(\operatorname{Int}\left(D^{\prime k}\right)\right) \cap \operatorname{Int}\left(D^{\prime k}\right)=\varnothing$;

- la suite $\left(D^{\prime k}\right)_{k \leq 0}$ converge vers $\{\alpha\}$ :

- la suite $\left(D^{\prime k}\right)_{k \geq 0}$ converge vers $\{\omega\}$.

Démonstration On peut épaissir chaque arc $\gamma^{\prime k}$ construit dans le Lemme 3.2 pour construire une suite $\left(D^{\prime k}\right)_{k \in \mathbb{Z}}$ de disques fermés disjoints deux à deux vérifiant toutes 
les conditions demandées sauf la quatrième. Quitte à diminuer les disques que l'on vient de construire, on peut cependant supposer que l'intérieur de chaque $D^{\prime k}$ est libre et donc que $f\left(D^{\prime k}\right) \cap D^{\prime k}=f\left(\partial D^{\prime k}\right) \cap \partial D^{\prime k}$. En effet, on peut écrire $D^{\prime k} \backslash\left\{z^{k}\right\}=$ $\bigsqcup_{t \in] 0,1]}\left(\partial D_{t}^{k} \backslash\left\{z^{k}\right\}\right)$, où $t \mapsto D_{t}^{k}$ est une fonction continue croissante de disques vérifiant:

- $z^{k} \in \partial D_{t}^{k}$

- $D_{1}^{k}=D^{\prime k}$;

- $\lim _{t \rightarrow 0} D_{t}^{k}=\left\{z^{k}\right\}$;

- $\operatorname{Int}\left(D_{t}^{k}\right)=\bigsqcup_{t^{\prime}<t}\left(\partial D_{t^{\prime}}^{k} \backslash\left\{z^{k}\right\}\right)$.

Il existe alors un plus petit réel $t \in] 0,1]$ tel que $D_{t}^{k}$ ne soit pas libre. On peut vérifier que son intérieur est nécessairement libre.

Continuons notre série de lemmes.

Lemme 3.4 L'une au moins des assertions suivantes est vraie.

(i) L'homéomorphisme $f$ est récurrent.

(ii) On peut construire une suite $\left(D^{k}\right)_{k \in \mathbb{Z}}$ de disques fermés disjoints deux à deux tels que:

- $z^{k} \in \operatorname{Int}\left(D^{k}\right)$;

- $D^{k} \cap \operatorname{Fix}(f)=\varnothing$;

- $f\left(D^{k}\right) \cap D^{k} \neq \varnothing$;

- $f\left(D^{k}\right) \cap D^{k^{\prime}}=f^{2}\left(D^{k}\right) \cap D^{k^{\prime}}=\varnothing$ si $k^{\prime}<k$;

- la suite $\left(D^{k}\right)_{k \leq 0}$ converge vers $\{\alpha\}$;

- la suite $\left(D^{k}\right)_{k \geq 0}$ converge vers $\{\omega\}$.

Démonstration Considérons une suite $\left(D^{\prime k}\right)_{k \in \mathbb{Z}}$ définie par le Lemme 3.3. Tout segment $\gamma$ joignant le point $z^{k}$ à un point $z^{\prime} \in \partial D^{\prime k}$ et contenu, sauf en ses extrémités, dans l'intérieur du disque $D^{\prime k}$, est libre. En effet $z_{k}$ n'étant pas fixe, le segment $f(\gamma)$ ne peut rencontrer $\gamma$ que si l'extrémité $z^{\prime}$ est fixe, égale à $f\left(z^{k}\right)=z^{k+1}$ ou égale à $f^{-1}\left(z^{k}\right)=z^{k-1}$. Le premier cas est impossible puisque $D^{\prime k} \cap \operatorname{Fix}(f)=\varnothing$, les deux autres cas le sont également pusique $D^{\prime k+1}$ et $D^{\prime k-1}$ sont disjoints de $D^{\prime k}$ et contiennent respectivement $z^{k+1}$ et $z^{k-1}$.

Choisissons $z^{\prime \prime k} \in \partial D^{\prime k} \cap f^{-1}\left(\partial D^{\prime k}\right)$, puis deux segments $\gamma_{-}^{k}$ et $\gamma_{+}^{k}$, le premier joignant $z^{\prime \prime k}$ à $z^{k}$, le second joignant $z^{k}$ à $f\left(z^{\prime \prime k}\right)$, tous deux contenus dans l'intérieur 
de $D^{\prime k}$, sauf aux extrémités, et ne se rencontrant qu'en $z^{k}$. Fixons $k^{\prime}<k$. L'orbite positive de chacun des segments $\gamma_{-}^{k^{\prime}}$ et $\gamma_{+}^{k^{\prime}}$ rencontre $\gamma_{-}^{k}$ et $\gamma_{+}^{k}$ (au moins au point $z^{k}$ ). Puisque tous ces segments sont libres, si l'orbite positive de $\gamma_{-}^{k}$ ou de $\gamma_{+}^{k}$ rencontre $\gamma_{-}^{k^{\prime}}$ ou $\gamma_{+}^{k^{\prime}}$, alors $f$ est récurrent, d'après la Proposition 1.4. Ainsi l'assertion (i) est vraie. Supposons maintenant que l'orbite positive de $\gamma_{-}^{k}$ et de $\gamma_{+}^{k}$ ne rencontre ni $\gamma_{-}^{k^{\prime}}$ ni $\gamma_{+}^{k^{\prime}}$, dès que $k^{\prime}<k$. Puisque

$$
\lim _{k \rightarrow-\infty} \gamma_{-}^{k} \gamma_{+}^{k}=\alpha, \lim _{k \rightarrow+\infty} \gamma_{-}^{k} \gamma_{+}^{k}=\omega,
$$

on peut trouver un disque fermé $D^{0}$, voisinage de $\gamma_{-}^{0} \gamma_{+}^{0}$, tel que

- $D^{0} \cap \operatorname{Fix}(f)=\varnothing$;

- $D^{0} \cap \gamma_{-}^{k} \gamma_{+}^{k}=f\left(D^{0}\right) \cap \gamma_{-}^{k} \gamma_{+}^{k}=f^{2}\left(D^{0}\right) \cap \gamma_{-}^{k} \gamma_{+}^{k}=\varnothing$, si $k<0$;

- $D^{0} \cap \gamma_{-}^{k} \gamma_{+}^{k}=f^{-1}\left(D^{0}\right) \cap \gamma_{-}^{k} \gamma_{+}^{k}=f^{-2}\left(D^{0}\right) \cap \gamma_{-}^{k} \gamma_{+}^{k}=\varnothing$, si $k>0$.

On a alors nécessairement:

- $z^{0} \in \operatorname{Int}\left(D^{0}\right)$;

- $f\left(D^{0}\right) \cap D^{0} \neq \varnothing$.

On peut choisir ensuite un disque fermé $D^{1}$, voisinage de $\gamma_{-}^{1} \gamma_{+}^{1}$, tel que

- $\quad D^{1} \cap \operatorname{Fix}(f)=\varnothing$;

- $D^{1} \cap \gamma_{-}^{k} \gamma_{+}^{k}=f\left(D^{1}\right) \cap \gamma_{-}^{k} \gamma_{+}^{k}=f^{2}\left(D^{1}\right) \cap \gamma_{-}^{k} \gamma_{+}^{k}=\varnothing$, si $k<0$;

- $D^{1} \cap D^{0}=f\left(D^{1}\right) \cap D^{0}=f^{2}\left(D^{1}\right) \cap D^{0}=\varnothing$;

- $D^{1} \cap \gamma_{-}^{k} \gamma_{+}^{k}=f^{-1}\left(D^{1}\right) \cap \gamma_{-}^{k} \gamma_{+}^{k}=f^{-2}\left(D^{1}\right) \cap \gamma_{-}^{k} \gamma_{+}^{k}=\varnothing$, si $k>1$.

On a alors:

- $z^{1} \in \operatorname{Int}\left(D^{1}\right)$;

- $f\left(D^{1}\right) \cap D^{1} \neq \varnothing$.

Par un simple procédé de récurrence on construit de cette façon notre suite $\left(D^{k}\right)_{k \in \mathbb{Z}}$.

Nous pouvons maintenant prouver la Proposition 3.1 en utilisant des arguments classiques dans le domaine. 
Démonstration de la Proposition 3.1 On peut bien sûr supposer que l'assertion (ii) du Lemme 3.4 est vérifiée et considérer une suite $\left(D^{k}\right)_{k \in \mathbb{Z}}$ définie par ce lemme. Là encore, en diminuant $D^{k}$ si nécessaire, on peut supposer que l'intérieur de chaque $D^{k}$ est libre. Fixons $z^{\prime \prime \prime} k \in \partial D^{k} \cap f^{-1}\left(\partial D^{k}\right)$, puis deux segments $\gamma_{-}^{\prime k}$ et $\gamma_{+}^{\prime k}$ le premier joignant $z^{\prime \prime \prime} k$ à $z^{k}$, le second joignant $z^{k}$ à $f\left(z^{\prime \prime \prime}\right)$ contenus dans l'intérieur de $D^{k}$, sauf en une extrémité, et ne se rencontrant qu'en $z^{k}$. Le segment $\gamma_{-}^{\prime k} \gamma_{+}^{\prime k}$ est un arc de translation. Si l'une des hypothèses de la Proposition 1.1 (le lemme de Brouwer) est vérifiée, alors $f$ est récurrent, d'après la Proposition 1.4. Supposons donc que pour tout $k \in \mathbb{Z}$, aucune de ces hypothèses n'est vérifée. Remarquons que $\gamma^{k}=\gamma_{+}^{\prime k} f\left(\gamma_{-}^{\prime k}\right)$ est alors un arc de translation joignant $z^{k}$ à $z^{k+1}$. Puisque $\gamma^{k} \subset D^{k} \cup f\left(D^{k}\right)$ et puisque $f$ se prolonge en un homéomorphisme de $\mathbf{D} \cup\{\alpha, \omega\}$, les deux propriétés de convergence de la Proposition 3.1 sont vérifiées. Si $k^{\prime}<k$, les ensembles $D^{k^{\prime}} \cup f\left(D^{k^{\prime}}\right)$ et $f\left(D^{k}\right) \cup f^{2}\left(D^{k}\right)$ sont disjoints. Ainsi toutes les conclusions de la proposition sont vérifiées.

Remarque Les trois premières assertions du Lemme 3.2 impliquent que chaque orbite $O_{i}$ est contenue dans une composante connexe de $\mathbf{D} \backslash$ Fix $(f)$ (on retrouve un cas particulier du théorème prouvé dans [3]). Les deux autres assertions ainsi que les hypothèses combinatoires sur les $\alpha_{i}$ et $\omega_{i}$ vérifées par $f$ impliquent en fait que les orbites $O_{i}$ sont contenues dans la même composante connexe de $\mathbf{D} \backslash \operatorname{Fix}(f)$.

Puisque nous voulons montrer que $f$ est récurrent, nous supposerons dorénavant que l'assertion (ii) de la Proposition 3.1 est vérifiée. Chaque segment $\gamma_{i}^{k}$ sera paramétré par $t \in[k, k+1]$. On a donc un paramétrage par $t \in \mathbb{R}$ de chaque arc $\Gamma_{i}=\prod_{k \in \mathbb{Z}} \gamma_{i}^{k}$ et on a $\lim _{t \rightarrow-\infty} \Gamma_{i}(t)=\alpha, \lim _{t \rightarrow+\infty} \Gamma_{i}(t)=\omega$. L'arc $\Gamma_{i}$ n'est pas nécessairement simple, contrairement aux arcs restreints $\left.\Gamma_{i}\right|_{[k, k+1]}$. Le fait que chaque $\gamma_{i}^{k}$ est un arc de translation qui joint $z_{i}^{k}$ à $z_{i}^{k+1}$ et dont l'image par $f$ ne rencontre aucun segment $\gamma_{i}^{k^{\prime}}, k^{\prime}<k$, nous donne:

Proposition 3.5 Pour tous réels $t$ et $t^{\prime}$, on a $f\left(\Gamma_{i}(t)\right) \neq \Gamma_{i}\left(t^{\prime}\right)$ si $t^{\prime} \leq t$.

\section{Mise sous forme générique}

Nous allons expliquer dans cette section comment perturber les $\gamma_{i}^{k}$, pour que les arcs $\Gamma_{i}$ soient en position "générique".

Proposition 4.1 On peut toujours supposer que les arcs $\gamma_{i}^{k}$ vérifient, en plus des propriétés énoncées dans la Proposition 3.1, les conditions suivantes: 
- $\gamma_{i}^{k} \cap O_{i}=\left\{z_{i}^{k}, z_{i}^{k+1}\right\}$;

- $\gamma_{i}^{k} \cap O_{i^{\prime}}=\varnothing$ si $i^{\prime} \neq i$;

- $\gamma_{i}^{k} \cap \gamma_{i^{\prime}}^{k^{\prime}}$ est fini si $\left(i^{\prime}, k^{\prime}\right) \notin\{(i, k-1),(i, k),(i, k+1)\}$;

- $\gamma_{i}^{k} \cap \gamma_{i^{\prime}}^{k^{\prime}} \cap \gamma_{i^{\prime \prime}}^{k^{\prime \prime}}=\varnothing$ si $(i, k),\left(i^{\prime}, k^{\prime}\right)$ et $\left(i^{\prime \prime}, k^{\prime \prime}\right)$ sont distincts.

Commençons par énoncer deux résultats préliminaires:

Lemme 4.2 Soit $\gamma$ un arc de translation d'un point $z \notin \operatorname{Fix}\left(f^{2}\right)$ et $\gamma^{\prime} \subset \operatorname{int}(\gamma)$ un segment. Il existe alors un voisinage $U$ de $\gamma^{\prime}$ tel que tout segment joignant $z$ à $f(z)$ à valeurs dans $\gamma \cup U$ est un arc de translation de $z$.

Démonstration Puisque

$$
f\left(\gamma^{\prime}\right) \cap \gamma^{\prime}=f\left(\gamma^{\prime}\right) \cap \gamma=\gamma^{\prime} \cap f(\gamma)=\varnothing,
$$

on peut choisir $U$ de telle façon que

$$
f(U) \cap U=f(U) \cap \gamma=U \cap f(\gamma)=\varnothing .
$$

On en déduit alors

$$
f(U \cup \gamma) \cap(U \cup \gamma)=f(\gamma) \cap \gamma=\{f(z)\} .
$$

Lemme 4.3 On peut construire sur $\mathbb{R}^{2}$ une famille $\left(X_{p}\right)_{p \in \mathbb{N}}$ de parties dénombrables denses, qui vérifient les propriétés suivantes, où $\Xi_{p}$ désigne l'ensemble des droites affines contenant au moins deux points de $X_{p}$ :

- si $p$ et $p^{\prime}$ sont distincts, alors $\Xi_{p} \cap \Xi_{p^{\prime}}=\varnothing$;

- si $p$, $p^{\prime}$ et $p^{\prime \prime}$ sont distincts, alors $\Delta \cap \Delta^{\prime} \cap \Delta^{\prime \prime}=\varnothing$ pour tout $\left(\Delta, \Delta^{\prime}, \Delta^{\prime \prime}\right) \in$ $\Xi_{p} \times \Xi_{p^{\prime}} \times \Xi_{p^{\prime \prime}}$

Démonstration On pose $X_{0}=\mathbb{Q}^{2}$. On suppose ensuite que la famille $\left(X_{p}\right)_{0 \leq p \leq P}$ a été construite jusqu'à l'ordre $P$ et vérifie les deux propriétés du Lemme 4.3. On va montrer qu'on peut adjoindre un ensemble $X_{P+1}$ pour que ces deux propriétés soient encore vérifiées. Remarquons que l'ensemble

$$
Y_{P}=\bigcup_{0 \leq p<p^{\prime} \leq P} \bigcup_{\Delta \in \Xi_{p}} \bigcup_{\Delta^{\prime} \in \Xi_{p^{\prime}}} \Delta \cap \Delta^{\prime}
$$

est dénombrable puisque la première condition est vraie jusqu'au rang $P$. Notons $\operatorname{Vect}_{\mathbb{Q}}(A)$ le $\mathbb{Q}$-espace vectoriel engendré par une partie $A \subset \mathbb{R}^{2}$ et $\mathbb{R} A$ l'ensemble des multiples réels d'élements de $A$. Si $A_{1}$ et $A_{2}$ sont deux parties de $\mathbb{R}^{2}$, notons 
$A_{1}+A_{2}$ l'ensemble des points $z \in \mathbb{R}^{2}$ qui s'écrivent $z=z_{1}+z_{2}$, où $z_{1} \in A_{1}$ et $z_{2} \in A_{2}$. L'ensemble

$$
Z_{P}=\operatorname{Vect}_{\mathbb{Q}}\left(Y_{P} \cup\left(\bigcup_{0 \leq p \leq P} X_{p}\right)\right)+\mathbb{R} \mathbb{Q}^{2}
$$

est une réunion dénombrable de droites affines (à pentes rationnelles) et donc un $F_{\sigma}$ d'intérieur vide. On choisit un point $x_{P+1} \notin Z_{P}$ et on pose $X_{P+1}=x_{P+1}+\mathbb{Q}^{2}$. Il est clair que $X_{P+1}$ est disjoint de $Z_{P}$. On vérifie aisément que la famille $\left(X_{p}\right)_{0 \leq p \leq P+1}$ vérifie les conditions demandées. En effet, toute droite $\Delta \in \Xi_{P+1}$ est à pente rationnelle et ne contient donc aucun élément de $X_{p}, p \leq P$. La première condition est donc vérifiée. Pour les mêmes raisons, $\Delta$ ne contient aucun point de $Y_{P}$, ce qui implique que la seconde condition est également vérifiée.

Avant de prouver la Proposition 4.1, remarquons d'abord que la famille $\left(\gamma_{i}^{k}\right)_{i, k}$ est localement finie: seul un nombre fini de ces arcs rencontrent une partie compacte donnée de $\mathbf{D}$. On utilisera implicitement ce fait dans la suite. Remarquons ensuite que l'on peut construire, par récurrence, une suite $\left(W_{i}^{k}\right)_{k \in \mathbb{Z}}$ de disques de sécurité:

- chaque $W_{i}^{k}$ est un disque ouvert contenant $\gamma_{i}^{k}$;

- $f\left(W_{i}^{k}\right) \cap W_{i}^{k^{\prime}}=\varnothing$ si $k^{\prime}<k$;

- la suite $\left(\bar{W}_{i}^{k}\right)_{k \leq 0}$ converge vers $\left\{\alpha_{i}\right\}$;

- la suite $\left(\bar{W}_{i}^{k}\right)_{k \geq 0}$ converge vers $\left\{\omega_{i}\right\}$.

Démonstration de la Proposition 4.1 Commençons par perturber les $\gamma_{i}^{k}$ pour que les deux premières assertions de la Proposition 4.1 soient vérifiées. Fixons $\gamma_{i}^{k}$. Les orbites $O_{i}$ sont disjointes deux à deux, fermées et discrètes. L'ensemble

$$
\gamma_{i}^{k} \cap\left(\left(\bigcup_{i^{\prime}} O_{i^{\prime}}\right) \backslash\left\{z_{i}^{k}, z_{i}^{k+1}\right\}\right)
$$

est donc fini et inclus dans un segment $\gamma^{\prime} \subset \operatorname{int}\left(\gamma_{i}^{k}\right)$. On choisit alors un voisinage $U$ de $\gamma^{\prime}$ satisfaisant le Lemme 4.2, inclus dans le disque de sécurité $W_{i}^{k}$ et tel que

$$
U \cap\left(\left(\bigcup_{i^{\prime}} O_{i^{\prime}}\right) \backslash\left\{z_{i}^{k}, z_{i}^{k+1}\right\}\right)=\gamma_{i}^{k} \cap\left(\left(\bigcup_{i^{\prime}} O_{i^{\prime}}\right) \backslash\left\{z_{i}^{k}, z_{i}^{k+1}\right\}\right) .
$$

On construit facilement un segment $\tilde{\gamma}_{i}^{k}$ dans $\gamma_{i}^{k} \cup U$ joignant $z_{i}^{k}$ à $z_{i}^{k+1}$ et évitant l'ensemble

$$
\gamma_{i}^{k} \cap\left(\left(\bigcup_{i^{\prime}} O_{i^{\prime}}\right) \backslash\left\{z_{i}^{k}, z_{i}^{k+1}\right\}\right) .
$$


Puisque dans cette construction, on ne touche pas aux autres arcs, on peut faire une simple récurrence et perturber tous les $\gamma_{i}^{k}$ pour qu'ils vérifient les deux premières conditions de la Proposition 4.1. Nous supposerons maintenant que ces deux conditions sont satisfaites et allons perturber de nouveaux les $\gamma_{i}^{k}$ pour que les deux dernières conditions soient également vérifiées.

On peut construire une famille de disques ouverts $\left(\Delta_{i}^{k}\right)_{i, k}$, disjoints deux à deux, tels que

- $\Delta_{i}^{k} \subset W_{i}^{k}$

- $z_{i}^{k} \in \Delta_{i}^{k}$;

- $\bar{\Delta}_{i}^{k} \cap \gamma_{i^{\prime}}^{k^{\prime}}=\varnothing \operatorname{si}\left(i^{\prime}, k^{\prime}\right) \notin\{(i, k-1),(i, k)\}$.

On choisit alors, pour tout $(i, k)$, un segment dans $\operatorname{int}\left(\gamma_{i}^{k}\right)$ avec une extrémité dans $\Delta_{i}^{k}$ et l'autre dans $\Delta_{i+1}^{k}$ puis un voisinage $U_{i}^{k} \subset W_{i}^{k}$ de ce segment qui vérifie le Lemme 4.2. On peut supposer de plus que $\bar{\Delta}_{i}^{k} \cap U_{i^{\prime}}^{k^{\prime}}=\varnothing$, si $\left(i^{\prime}, k^{\prime}\right) \notin\{(i, k-1),(i, k)\}$.

On peut trouver une famille $\left(X_{i}^{k}\right)_{i, k}$ de parties dénombrables denses de $\mathbb{R}^{2}$ qui vérifient les propriétés indiquées dans le Lemme 4.3. On construit ensuite une famille $\left(\widetilde{\gamma}_{i}^{k}\right)_{i, k}$ de segments, tels que $\tilde{\gamma}_{i}^{k}$ joint $z_{i}^{k}$ à $z_{i}^{k+1}$, est à valeurs dans $\gamma_{i}^{k} \cup U_{i}^{k}$ et s'écrit $\tilde{\gamma}_{i}^{k}=\tilde{\gamma}_{i}^{k, 1} \tilde{\gamma}_{i}^{k, 2} \tilde{\gamma}_{i}^{k, 3}$, où $\tilde{\gamma}_{i}^{k, 1}$ (resp. $\tilde{\gamma}_{i}^{k, 2}, \tilde{\gamma}_{i}^{k, 3}$ ) est un segment à valeurs dans $\Delta_{i}^{k}$, (resp. $U_{i}^{k}, \Delta_{i}^{k+1}$ ), et où $\tilde{\gamma}_{i}^{k, 2}$ est affine par morceaux avec des sommets dans $X_{i}^{k}$.

Si $\left(i^{\prime}, k^{\prime}\right) \notin\{(i, k-1),(i, k),(i, k+1)\}$, alors $\tilde{\gamma}_{i}^{k} \cap \tilde{\gamma}_{i^{\prime}}^{k^{\prime}}=\tilde{\gamma}_{i}^{k, 2} \cap \tilde{\gamma}_{i^{\prime}}^{k^{\prime}, 2}$ est fini. De même, si $(i, k),\left(i^{\prime}, k^{\prime}\right)$ et $\left(i^{\prime \prime}, k^{\prime \prime}\right)$ sont distincts, alors

$$
\tilde{\gamma}_{i}^{k} \cap \tilde{\gamma}_{i^{\prime}}^{k^{\prime}} \cap \tilde{\gamma}_{i^{\prime \prime}}^{k^{\prime \prime}}=\tilde{\gamma}_{i}^{k, 2} \cap \tilde{\gamma}_{i^{\prime}}^{k^{\prime}, 2} \cap \tilde{\gamma}_{i^{\prime \prime}}^{k^{\prime \prime}, 2}=\varnothing .
$$

On supposera dorénavant que les $\gamma_{i}^{k}$ vérifient les propriétés énoncées dans les propositions 3.1 et 4.1 .

Proposition 4.4 Les arcs $\Gamma_{i}$ vérifient les propriétés suivantes:

- tout point $z_{i}^{k}$ est un point simple de $\Gamma_{i}$;

- les arcs $\Gamma_{i}$ n'ont pas de points triples;

- $\Gamma_{i} \cap O_{i^{\prime}}=\varnothing$, si $i \neq i^{\prime}$;

- $\Gamma_{i} \cap \Gamma_{i^{\prime}}$ est fini, si $i \neq i^{\prime}$;

- $\Gamma_{i} \cap \Gamma_{i^{\prime}}$ ne contient aucun point double ni de $\Gamma_{i}$, ni de $\Gamma_{i^{\prime}}$, si $i \neq i^{\prime}$;

- $\Gamma_{i} \cap \Gamma_{i^{\prime}} \cap \Gamma_{i^{\prime \prime}}=\varnothing$ si $i$, $i^{\prime}$ et $i^{\prime \prime}$ sont distincts. 
Démonstration Il s'agit de conséquences immédiates des points suivants:

- les $\operatorname{arcs} \gamma_{i}^{k}=\left.\Gamma_{i}\right|_{[k, k+1]}$ sont des arcs simples;

- les $\operatorname{arcs} \gamma_{i}^{k}$ vérifient les conditions de la Proposition 4.1;

- $\lim _{k \rightarrow-\infty} \gamma_{i}^{k}=\left\{\alpha_{i}\right\}$;

- $\lim _{k \rightarrow+\infty} \gamma_{i}^{k}=\left\{\omega_{i}\right\}$;

- les $2 n$ points $\omega_{i}, \alpha_{i}, i \in \mathbb{Z} / n \mathbb{Z}$, sont distincts.

\section{Démonstration du lemme topologique fondamental}

Nous prouverons dans ce paragraphe le résultat-clé de l'article (Proposition 5.1). Il s'agit d'un résultat purement topologique qui utilise que le fait que $\Gamma_{i}$ joint $\alpha_{i}$ à $\omega_{i}$ ainsi que les hypothèses combinatoires (ii) et (iii) du Théorème 0.1 . Le résultat est encore vrai sans les hypothèses de généricité décrites par la Proposition 4.4. Nous n'écrirons cependant la preuve que dans le cas où ces hypothèses sont vérifiées, beaucoup d'arguments se simplifiant.

Nous noterons $\Gamma_{i}^{\leq t}$ (resp. $\Gamma_{i}^{<t}$ ) la restriction de $\Gamma_{i}$ à $\left.]-\infty, t\right]$ (resp. $]-\infty, t[$ ) et nous définirons de façon analogue $\Gamma_{i}^{\geq t}$ et $\Gamma_{i}^{>t}$. Nous ferons, en prenant les précautions usuelles, l'abus de langage qui consiste à identifier un arc à son image. Pour des questions d'homogénéité d'écriture, nous écrirons $\Gamma_{i}^{t}$ au lieu de $\Gamma_{i}(t)$. Nous définirons l'ensemble des configurations $\mathcal{T}=\mathbb{R}^{\mathbb{Z} / n \mathbb{Z}}$ et pour tout $i_{0} \in \mathbb{Z} / n \mathbb{Z}$, nous écrirons $p_{i_{0}}: T=\left(t_{i}\right)_{i \in \mathbb{Z} / n \mathbb{Z}} \mapsto t_{i_{0}}$ pour la $i_{0}$-ème projection.

Si $K$ est un entier assez grand et si $i$ et $i^{\prime}$ sont distincts, alors

$$
\left.\left.\Gamma_{i}(]-\infty,-K\right]\right) \cap \Gamma_{i^{\prime}}=\Gamma_{i}\left(\left[K,+\infty[) \cap \Gamma_{i^{\prime}}=\varnothing .\right.\right.
$$

Nous fixons un tel entier $K$ jusqu'à la fin de l'article.

Proposition 5.1 Il existe $T=\left(t_{i}\right)_{i \in \mathbb{Z} / n \mathbb{Z}} \in \mathcal{T}$ tel que:

(A) les ensembles $\Gamma_{i}^{<t_{i}}, i \in \mathbb{Z} / n \mathbb{Z}$, sont disjoints deux à deux;

(B) pour tout $i \in \mathbb{Z} / n \mathbb{Z}$, il existe $i^{\prime} \notin\{i-1, i\}$ tel que $\Gamma_{i}^{\leq t_{i}} \cap \Gamma_{i^{\prime}}^{\leq t_{i^{\prime}}} \neq \varnothing$.

Remarquons que si les $t_{i}$ sont tous très petits, la condition (A) est vérifiée mais pas la condition (B). Si, par contre, les $t_{i}$ sont tous très grands, c'est (B) qui est vérifiée, mais pas (A). Nous devons donc chercher une configuration critique, correspondant à une situation de connexité limite. Nous utiliserons un procédé de type minimax. 
Pour tout $T=\left(t_{i}\right)_{i \in \mathbb{Z} / n \mathbb{Z}} \in \mathcal{T}$, notons $\Sigma(T)$ l'ensemble des $i \in \mathbb{Z} / n \mathbb{Z}$ tels qu'il existe $i^{\prime} \notin\{i-1, i\}$ vérifiant $\Gamma_{i}^{\leq t_{i}} \cap \Gamma_{i^{\prime}}^{\leq t_{i^{\prime}}} \neq \varnothing$ et appelons ordre de $T$ le cardinal de $\Sigma(T)$. D'après les hypothèses (ii) et (iii) du Théorème 0.1 , on peut remarquer que si $i \in \Sigma(T)$ et si $\Gamma_{i}^{\leq t_{i}}$ rencontre $\Gamma_{i^{\prime}}^{\leq t_{i^{\prime}}}, i^{\prime} \notin\{i-1, i\}$, alors les arcs $\Gamma_{i-1}^{\leq-K}$ et $\Gamma_{i-1}^{\geq K}$ sont séparés dans D par $\Gamma_{i}^{\leq t_{i}} \cup \Gamma_{i^{\prime}}^{\leq t_{i^{\prime}}}$. Notons également $\mathcal{T}_{*}$ l'ensemble des configurations vérifiant (A). Remarquons que c'est une partie fermée de $\mathcal{T}$.

Lemme 5.2 Soit $T=\left(t_{i}\right)_{i \in \mathbb{Z} / n \mathbb{Z}} \in \mathcal{T}_{*}$.

(i) Pour tout $i \in \Sigma(T)$, on a $t_{i}>-K$ et $t_{i-1}<K$.

(ii) Les configurations

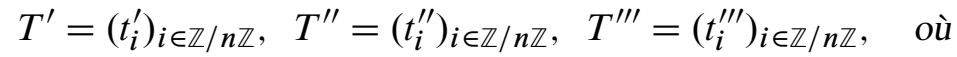

$$
\begin{aligned}
& t_{i}^{\prime}=\min \left(t_{i}, K\right), \quad t_{i}^{\prime \prime}=\max \left(t_{i},-K\right), t_{i}^{\prime \prime \prime}=\min \left(t_{i}^{\prime \prime}, K\right)=\max \left(t_{i}^{\prime},-K\right),
\end{aligned}
$$

appartiennent à $\mathcal{T}_{*}$. De plus on a

$$
\Sigma\left(T^{\prime}\right)=\Sigma\left(T^{\prime \prime}\right)=\Sigma\left(T^{\prime \prime \prime}\right)=\Sigma(T) .
$$

Démonstration Soit $i \in \Sigma(T)$ et $i^{\prime} \notin\{i-1, i\}$ tel que $\Gamma_{i}^{\leq t_{i}} \cap \Gamma_{i^{\prime}}^{\leq t_{i^{\prime}}} \neq \varnothing$. L'égalité $t_{i}>-K$ est une conséquence de la définition de $K$. Prouvons par l'absurde que $t_{i-1}<K$. Dans le cas contraire, l'arc $\Gamma_{i-1}=\Gamma_{i-1}^{<t_{i-1}} \Gamma_{i-1}^{\geq t_{i-1}}$ serait disjoint de $\Gamma_{i}^{<t_{i}}$ et de $\Gamma_{i^{\prime}}^{<t_{i^{\prime}}}$, il séparerait ces deux arcs. Les deux $\operatorname{arcs} \Gamma_{i}^{\leq t_{i}}$ et $\Gamma_{i^{\prime}}^{\leq t_{i^{\prime}}}$ auraient donc une extrémité commune $\Gamma_{i}^{t_{i}}=\Gamma_{i^{\prime}}^{t_{i}^{\prime}}$ située sur $\Gamma_{i-1}$. Ceci contredit la dernière propriété de la Proposition 4.4.

Il est évident que $T^{\prime} \in \mathcal{T}_{*}$ et que $\Sigma\left(T^{\prime}\right) \subset \Sigma(T)$ puisque $t_{i}^{\prime} \leq t_{i}$, pour tout $i \in \mathbb{Z} / n \mathbb{Z}$. Soit $i \in \Sigma(T)$ et $i^{\prime} \notin\{i-1, i\}$ tel que $\Gamma_{i}^{\leq t_{i}} \cap \Gamma_{i^{\prime}}^{\leq t_{i^{\prime}}} \neq \varnothing$. Puisque $\Gamma_{i}^{\geq K} \cap \Gamma_{i^{\prime}}=$ $\Gamma_{i^{\prime}}^{\geq K} \cap \Gamma_{i}=\varnothing$, on en déduit que $\Gamma_{i}^{\leq t_{i}^{\prime}} \cap \Gamma_{i^{\prime}}^{\leq t_{i^{\prime}}^{\prime}} \neq \varnothing$ et donc que $i \in \Sigma\left(T^{\prime}\right)$.

Puisque $\Gamma_{i}^{\leq-K} \cap \Gamma_{i^{\prime}}=\varnothing$, si $i^{\prime} \neq i$, on sait que $T^{\prime \prime} \in \mathcal{T}_{*}$. On sait également que $\Sigma(T) \subset \Sigma\left(T^{\prime \prime}\right)$ puisque $t_{i} \leq t_{i}^{\prime \prime}$, pour tout $i \in \mathbb{Z} / n \mathbb{Z}$. Soit $i \in \Sigma\left(T^{\prime \prime}\right)$ et $i^{\prime} \notin\{i-1, i\}$ tel que $\Gamma_{i}^{\leq t_{i}^{\prime \prime}} \cap \Gamma_{i^{\prime}}^{\leq t_{i^{\prime \prime}}^{\prime \prime}} \neq \varnothing$. Puisque $\Gamma_{i}^{\leq-K} \cap \Gamma_{i^{\prime}}=\Gamma_{i^{\prime}}^{\leq-K} \cap \Gamma_{i}=\varnothing$, on en déduit que $t_{i}^{\prime \prime}=t_{i}$ et $t_{i^{\prime}}^{\prime \prime}=t_{i^{\prime}}$, puis que $i \in \Sigma(T)$.

On en déduit finalement que $T^{\prime \prime \prime} \in \mathcal{T}_{*}$ et que $\Sigma\left(T^{\prime \prime \prime}\right)=\Sigma(T)$. Remarquons que $\left|t_{i}^{\prime \prime \prime}\right| \leq K$, pour tout $i \in \mathbb{Z} / n \mathbb{Z}$.

Démontrer la Proposition 5.1, c'est trouver une configuration $T \in \mathcal{T}_{*}$ d'ordre $n$. Commençons d'abord par le résultat suivant: 
Lemme 5.3 Il existe une configuration $T=\left(t_{i}\right)_{i \in \mathbb{Z} / n \mathbb{Z}} \in \mathcal{T}_{*}$ d'ordre au moins $n-1$.

Démonstration Fixons $i \in \mathbb{Z} / n \mathbb{Z}$. Utilisant les hypothèses (ii) et (iii) du Théorème 0.1 , on sait que $\Gamma_{i}^{\leq-K}$ et $\Gamma_{i}^{\geq K}$ sont disjoints de $\Gamma_{i-1}$ et séparés par cet arc. On peut donc définir le premier instant $t_{i}$ où $\Gamma_{i}$ rencontre $\Gamma_{i-1}$. D'après la Proposition 4.4, le point $\Gamma_{i}^{t_{i}}$ est un point simple de $\Gamma_{i-1}$. On écrit $\Gamma_{i}^{t_{i}}=\Gamma_{i-1}^{t_{i-1}}$. Remarquons que $\Gamma_{i}^{<t_{i}} \cap \Gamma_{i-1}^{<t_{i-1}}=\varnothing$ et que $\Gamma_{i}^{\leq t_{i}} \cup \Gamma_{i-1}^{\leq t_{i-1}}$ sépare $\Gamma_{i-2}^{\leq-K}$ de $\Gamma_{i-2}^{\geq K}$. On peut donc définir le premier instant $t_{i-2}$ où $\Gamma_{i-2}$ rencontre $\Gamma_{i}^{\leq t_{i}} \cup \Gamma_{i-1}^{\leq t_{i-1}}$. On peut continuer le raisonnement et définir par récurrence sur $r \in\{2, \ldots, n-1\}$ le premier instant $t_{i-r}$ où $\Gamma_{i-r}$ rencontre $\bigcup_{0 \leq s \leq r-1} \Gamma_{i-s}^{\leq t_{i-s}}$. La configuration $T=\left(t_{i}\right)_{i \in \mathbb{Z} / n \mathbb{Z}}$ vérifie (A) et $\Sigma(T)$ contient $\{i-n+2, \ldots, i-1\}$. Remarquons maintenant que $\Sigma(T)$ contient $i$ si $\Gamma_{i+1}^{t_{i+1}}=\Gamma_{i-n+1}^{t_{i-n+1}} \in \Gamma_{i}^{\leq t_{i}}$. Si, par contre, $\Gamma_{i+1}^{t_{i+1}} \notin \Gamma_{i}^{\leq t_{i}}$, il existe $j \in\{i-n+2, \ldots, i-1\}$ tel que $\Gamma_{i+1}^{t_{i+1}} \in \Gamma_{j}^{\leq t_{j}}$. On en déduit que $i+1 \in \Sigma(T)$. La configuration $T$ est donc au moins d'ordre $n-1$ (les deux cas sont illustrés sur la Figure 5).
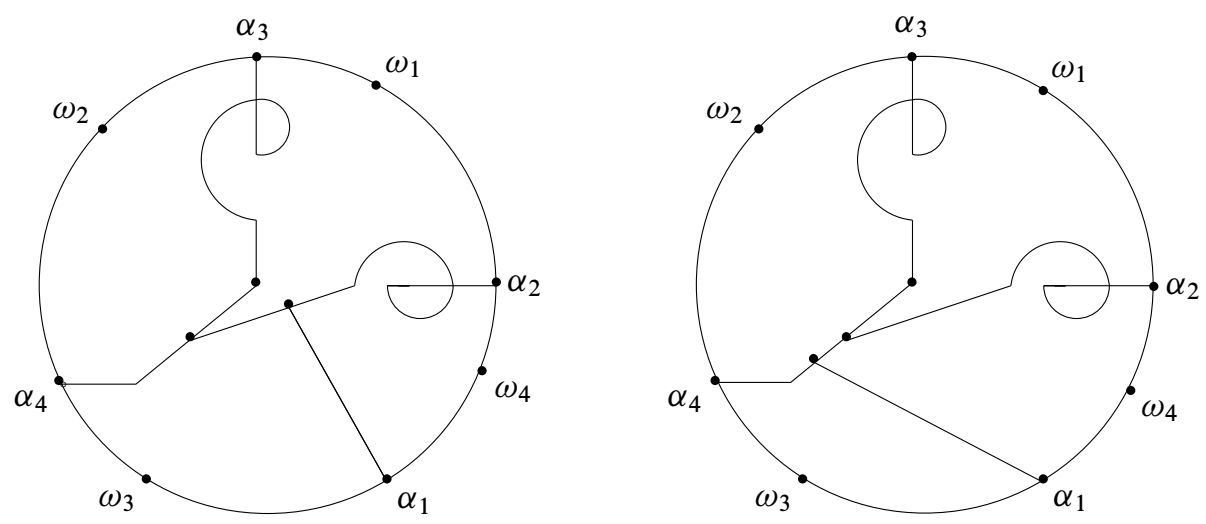

Figure 5

Pour des raisons d'écriture, il sera plus commode dans la suite de la Section 5 d'indexer $\mathcal{T}$ par $j \in\{1, \ldots, n\}$. Quitte à changer les indices, on peut supposer, d'après le lemme précédent, qu'il existe $T \in \mathcal{T}_{*}$ telle que $j \in \Sigma(T)$, si $j \neq n$. D'après le Lemme 5.2, on peut toujours supposer que $\left|t_{j}\right| \leq K$, pour tout $j \in\{1, \ldots, n\}$. On notera $\mathcal{T}_{-1} \subset \mathcal{T}_{*}$ l'ensemble des configurations vérifiant ces deux propriétés. Remarquons que c'est une partie compacte de $\mathcal{T}$. On en déduit que l'ensemble $\mathcal{T}_{0}$ formé des configurations $T \in \mathcal{T}_{-1}$ où $p_{n}$ atteint son maximum est une partie compacte non vide. On notera $t_{n}^{*}$ ce maximum. Nous voulons montrer qu'il existe une configuration d'ordre $n$ dans $\mathcal{T}_{-1}$. Ce sera une conséquence du Lemme 5.4 qui va suivre (et de la finitude de $\{1, \ldots, n\})$. Le principe est un raisonnement par l'absurde, on suppose qu'il n'y a pas 
de configuration d'ordre $n$ dans $\mathcal{T}_{-1}$ et on construira alors par récurrence une suite (infinie !) décroissante de parties compactes $\left(\mathcal{T}_{r}\right)_{r \geq 0}$ de $\mathcal{T}$ définies par les valeurs de $r+1$ projections. Nous allons commencer par initier la récurrence, pour faire ressortir les idées, puis nous énoncerons et démontrerons la construction générale (Lemme 5.4).

Fixons $T=\left(t_{j}\right)_{1 \leq j \leq n}$ dans $\mathcal{T}_{0}$ et montrons le résultat suivant:

Le point $\Gamma_{n}^{t_{n}^{*}}$ appartient à un arc $\Gamma_{j_{1}}^{\leq t_{j_{1}}}, j_{1} \neq n$, cet entier $j_{1}$ est unique, il est égal à $n-1$ et on a $\Gamma_{n}^{t_{n}^{*}} \in \Gamma_{n-1}^{<t_{n-1}}$.

D'après la première assertion du Lemme 5.2 appliqué à $j=1 \in \Sigma(T)$, on sait que $t_{n}=t_{n}^{*}<K$. D'après la Proposition 4.4, on sait qu'il existe $\left.t_{n}^{\prime} \in\right] t_{n}^{*}, K[$ tel que $\left.\Gamma_{n}\right|_{\left.] t_{n}^{*}, t_{n}^{\prime}\right]}$ ne rencontre aucun arc $\Gamma_{j}, j \neq n$. La configuration $T^{\prime}$ obtenue à partir de $T$ en remplaçant $t_{n}^{*}$ par $t_{n}^{\prime}$ n'est pas dans $\mathcal{T}_{-1}$, puisque $t_{n}^{*}$ est le maximum de $p_{n}$ sur $\mathcal{T}_{-1}$. Or les coordonnées de $T^{\prime}$ sont toutes entre $-K$ et $K$ et on a bien évidemment $\Sigma(T) \subset \Sigma\left(T^{\prime}\right)$. On en déduit que $T^{\prime}$ ne vérifie pas la condition (A). Ceci implique que $\Gamma_{n}^{t_{n}^{*}}$ appartient à un arc $\Gamma_{j_{1}}^{\leq t_{j_{1}}}, j_{1} \neq n$. La propriété 4.4 nous dit que l'entier $j_{1}$ est unique et que $\Gamma_{n}^{t_{n}^{*}}$ est un point simple de $\Gamma_{j_{1}}$. Le fait que $T^{\prime}$ ne vérifie pas la condition (A) implique en fait que $\Gamma_{n}^{t_{n}^{*}}$ n'est pas l'extrémité $\Gamma_{j_{1}}^{t_{j_{1}}}$ de $\Gamma_{j_{1}}^{\leq t_{j_{1}}}$ et appartient donc à $\Gamma_{j_{1}}^{<t_{j_{1}}}$. Le fait que $n \notin \Sigma(T)$ implique que $j_{1}=n-1$.

On définit alors

$$
\begin{aligned}
t_{n-1}^{*} & =\left.\min p_{n-1}\right|_{\mathcal{T}_{0}}, \\
\mathcal{T}_{1} & =\left\{T \in \mathcal{T}_{0} \mid p_{n-1}(T)=t_{n-1}^{*}\right\} .
\end{aligned}
$$

L'ensemble $\mathcal{T}_{1}$ est non vide, on choisit donc $T=\left(t_{j}\right)_{1 \leq j \leq n} \in \mathcal{T}_{1}$. Montrons le résultat suivant:

Le point $\Gamma_{n-1}^{t_{n-1}^{*}}$ appartient à un arc $\Gamma_{j_{2}}^{\leq t_{j_{2}}}, j_{2} \neq n-1$, cet entier $j_{2}$ est unique et vérifie $j_{2}<n-1$.

Puisque $n-1 \in \Sigma(T)$ on sait, d'après le Lemme 5.2, que $t_{n-1}=t_{n-1}^{*}>-K$. D'après la Proposition 4.4, on sait qu'il existe $\left.t_{n-1}^{\prime} \in\right]-K, t_{n-1}^{*}\left[\right.$ tel que $\left.\Gamma_{n-1}\right|_{\left.] t_{n-1}^{\prime}, t_{n-1}^{*}\right]}$ ne rencontre aucun arc $\Gamma_{j}, j \neq n-1$. Par définition de $t_{n-1}^{*}$, on sait que la configuration $T^{\prime}$ obtenue à partir de $T$ en remplaçant $t_{n-1}^{*}$ par $t_{n-1}^{\prime}$ n'appartient pas à $\mathcal{T}_{0}$. Elle vérifie bien évidemment (A), puisque c'est le cas de $T$. Puisque les coordonnées de $T^{\prime}$ sont toutes entre $-K$ et $K$ et puisque sa $n$-ième coordonnée est $t_{n}^{*}$, c'est donc qu'il existe $j \neq n$ tel que $j \notin \Sigma\left(T^{\prime}\right)$. On en déduit que $\Gamma_{n-1}^{t_{n-1}^{*}}$ appartient à un arc $\Gamma_{j_{2}}^{\leq t_{j_{2}}}$, $j_{2} \neq n-1$, que $j_{2}$ est unique et que l'entier $j \neq n$ qui n'est plus dans $\Sigma\left(T^{\prime}\right)$ est soit 
$n-1$ soit $j_{2}$. Or on a vu précédemment que le point $\Gamma_{n}^{t_{n}^{*}}$ n'est pas l'extrémité $\Gamma_{n-1}^{t_{n-1}^{*}}$. Il appartient donc à $\Gamma_{n-1}^{<t_{n-1}^{*}}$ et par conséquent appartient à $\Gamma_{n-1}^{\leq t_{n-1}^{\prime}}$. On en déduit que $n-1 \in \Sigma\left(T^{\prime}\right)$. C'est donc l'entier $j_{2}$ qui n'est pas dans $\Sigma\left(T^{\prime}\right)$, ainsi $j_{2} \neq n$. On en déduit que $j_{2}<n-1$.

On définit alors

$$
\begin{aligned}
& t_{j_{2}}^{*}=\max p_{j_{2}} \mid \mathcal{T}_{1}, \\
& \mathcal{T}_{2}=\left\{T \in \mathcal{T}_{1} \mid p_{j_{2}}(T)=t_{j_{2}}^{*}\right\} .
\end{aligned}
$$

L'ensemble $\mathcal{T}_{2}$ est non vide, on choisit donc $T=\left(t_{j}\right)_{1 \leq j \leq n} \in \mathcal{T}_{2}$. Montrons la propriété suivante:

le point $\Gamma_{j_{2}}^{t_{j_{2}}^{*}}$ appartient à un arc $\Gamma_{j_{3}}^{\leq t_{j_{3}}}, j_{3} \neq j_{2}$, cet entier $j_{3}$ est unique, il est égal à $j_{2}-1$ et on $a \Gamma_{j_{2}}^{t_{j_{2}}^{*}} \in \Gamma_{j_{2}-1}^{<t_{j_{2}-1}}$.

On sait que $j_{2} \neq n-1$ et donc que $j_{2}+1 \in \Sigma(T)$. On en déduit que $t_{j_{2}}=t_{j_{2}}^{*}<K$, d'après le Lemme 5.2. Utilisant le raisonnement fait plus haut (qui utilise la maximalité de $t_{2}^{*}$ ) on sait qu'il existe $j_{3} \neq j_{2}$, unique, tel que $\Gamma_{j_{2}}^{t_{j_{2}}^{*}} \in \Gamma_{j_{3}}^{\leq t_{j_{3}}}$ et que $\Gamma_{j_{2}}^{t_{j_{2}}^{*}} \neq \Gamma_{j_{3}}^{t_{j_{3}}}$. On écrit alors $\Gamma_{j_{2}}^{t_{j_{2}}^{*}}=\Gamma_{j_{3}}^{t^{\prime \prime} j_{3}}$. Notons $T^{\prime}=\left(t_{j}^{\prime}\right)_{1 \leq j \leq n}$ la configuration obtenue à partir de $T$ en remplaçant $t_{n-1}^{*}$ par $\left.t_{n-1}^{\prime} \in\right]-K, t_{n-1}^{*}\left[\right.$, où $\left.\Gamma_{n-1}\right|_{\left.] t_{n-1}^{\prime}, t_{n-1}^{*}\right]}$ ne rencontre aucun arc $\Gamma_{j}, j \neq n-1$. Nous avons vu plus haut que $j_{2} \notin \Sigma\left(T^{\prime}\right)$. Remarquons que $t_{j_{3}}^{\prime \prime} \leq t_{j_{3}}^{\prime}$ dans le cas éventuel où $j_{3}$ serait égal à $n-1$. Dans le cas contraire nous avons l'égalité $t_{j_{3}}^{\prime \prime}=t_{j_{3}}^{\prime}$. Nous en déduisons que $\Gamma_{j_{2}}^{\leq t_{j_{2}}^{\prime}} \cap \Gamma_{j_{3}}^{\leq t_{j_{3}}^{\prime}} \neq \varnothing$ et par conséquent que $j_{3}$ est nécessairement égal à $j_{2}-1$. Remarquons également que les seuls arcs $\Gamma_{j}^{\leq t_{j}}, j \neq j_{2}$, rencontrés par $\Gamma_{j_{2}}^{\leq t_{j_{2}}^{*}}$ sont $\Gamma_{n-1}^{\leq t_{n-1}^{*}}$ et $\Gamma_{j_{3}}^{\leq t_{j_{3}}^{*}}$ (voir Figure 6).

En continuant ce processus, nous allons montrer:

Lemme 5.4 Si $\mathcal{T}_{-1}$ ne contient aucune configuration d'ordre $n$, on peut construire:

- une suite strictement décroissante d'entiers positifs $\left(j_{r}\right)_{r \geq 0}$, vérifiant $j_{0}=n$ et $j_{2 r+1}=j_{2 r}-1$;

- une suite de réels $\left(t_{j_{r}}^{*}\right)_{r \geq 0}$;

- une suite décroissante de parties compactes $\left(\mathcal{T}_{r}\right)_{r \geq 0}$ de $\mathcal{T}$;

uniquement définies par les propriétés suivantes:

(i) $t_{j_{2 r}}^{*}=\max p_{j_{2 r}} \mid \mathcal{T}_{2 r-1}$ et $\mathcal{T}_{2 r}=\left\{T \in \mathcal{T}_{2 r-1} \mid p_{j_{2 r}}(T)=t_{j_{2 r}}^{*}\right\}$;

(ii) $t_{j_{2 r+1}}^{*}=\min p_{j_{2 r+1}} \mid \mathcal{T}_{2 r}$ et $\mathcal{T}_{2 r+1}=\left\{T \in \mathcal{T}_{2 r} \mid p_{j_{2 r+1}}(T)=t_{j_{2 r+1}}^{*}\right\}$; 


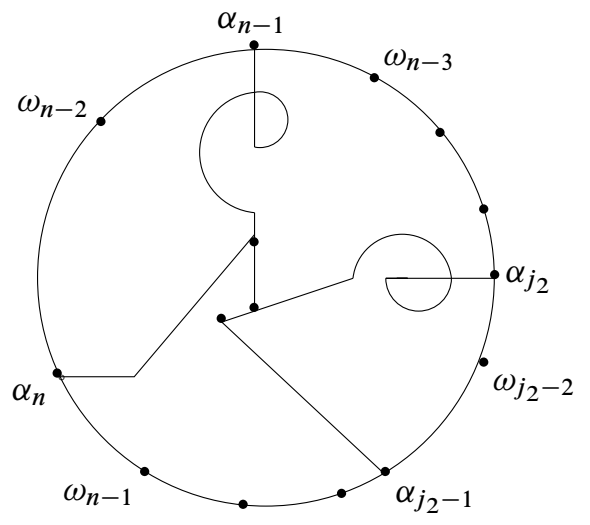

Figure 6

(iii) il existe un unique entier $j_{2 r+2} \neq j_{2 r+1}$ dans $\{1, \ldots, n\}$ tel que $\Gamma_{j_{2 r+1}}^{t_{2 r+1}^{*}} \in$ $\Gamma_{j_{2 r+2}}$.

De plus, on a:

(iv) si $T=\left(t_{j}\right)_{1 \leq j \leq n} \in \mathcal{T}_{2 r}$, l'arc $\Gamma_{j_{2 r}}^{\leq t_{j_{2 r}}}=\Gamma_{j_{2 r}}^{\leq t_{j_{2 r}}^{*}}$ est disjoint de $\Gamma_{j}^{\leq t_{j}}$ si $j \notin$ $\left\{j_{2 r-1}, j_{2 r}, j_{2 r+1},\right\}$ (si $j \notin\{n-1, n\}$ dans le cas où $r=0$ ) ainsi que de $\Gamma_{j_{2 r-1}}^{<t_{j_{2 r-1}}}=\Gamma_{j_{2 r-1}}^{<t_{j_{2 r-1}}^{*}}$;

(v) si $T=\left(t_{j}\right)_{1 \leq j \leq n} \in \mathcal{T}_{2 r}$, le point $\Gamma_{j_{2 r}}^{t_{j_{2 r}}}=\Gamma_{j_{2 r}}^{t_{j_{2 r}}^{*}}$ appartient à $\Gamma_{j_{2 r+1}}^{<t_{j_{2 r+1}}}$;

(vi) si $T=\left(t_{j}\right)_{1 \leq j \leq n} \in \mathcal{T}_{2 r+1}$, le point $\Gamma_{j_{2 r+1}}^{t_{j_{2 r+1}}}=\Gamma_{j_{2 r+1}}^{t_{j_{2 r+1}}^{*}}$ appartient à $\Gamma_{j_{2 r+2}}^{\leq t_{j_{2 r+2}}}$.

Démonstration On peut supposer que les ensembles $\mathcal{T}_{s}$ ont été définis jusqu'à l'ordre $2 r-1$, que les entiers $\left(j_{s}\right)$ ont été définis jusqu'à l'ordre $2 r$ et que les assertions du théorème sont vérifiées jusqu'à cet ordre. C'est le cas, on l'a vu, pour $r=1$. On définit alors $t_{j_{2 r}}^{*}$ et $\mathcal{T}_{2 r}$ par la condition (i). On pose ensuite $j_{2 r+1}=j_{2 r}-1$ et on définit $t_{j_{2 r+1}}^{*}$ et $\mathcal{T}_{2 r+1}$ par la condition (ii). On va commencer par montrer que (iv), puis (v), sont vérifiées. On montrera ensuite que c'est également le cas de (iii) et (vi). Enfin, on prouvera que $j_{2 r+2}$ est strictement plus petit que $j_{2 r+1}$.

Preuve de (iv) Fixons $T=\left(t_{j}\right)_{1 \leq j \leq n} \in \mathcal{T}_{2 r}$. Puisque $j_{2 r-1} \neq n$ on sait que $j_{2 r-1} \in$ $\Sigma(T)$ et donc que $t_{j_{2 r-1}}=t_{j_{2 r-1}}^{*}>-K$, d'après le Lemme 5.2. Choisissons $t_{j_{2 r-1}}^{\prime} \in$

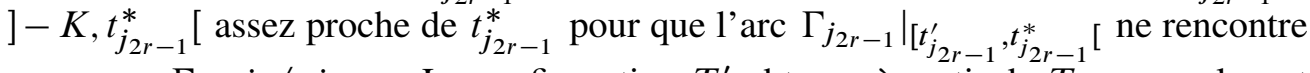
aucun arc $\Gamma_{j}, j \neq j_{2 r-1}$. La configuration $T^{\prime}$ obtenue à partir de $T$ en remplaçant $t_{j_{2 r-1}}^{*}$ par $t_{j_{2 r-1}}^{\prime}$ n'étant plus dans $\mathcal{T}_{2 r-2}$, il existe donc $j \neq n$ qui n'est plus dans $\Sigma(T)$. 


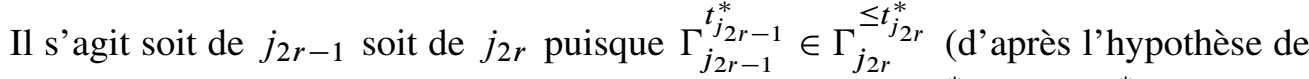

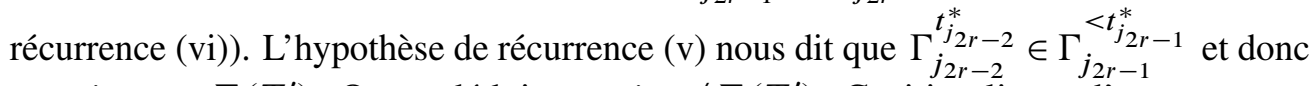
que $j_{2 r-1} \in \Sigma\left(T^{\prime}\right)$. On en déduit que $j_{2 r} \notin \Sigma\left(T^{\prime}\right)$. Ceci implique, d'une part que

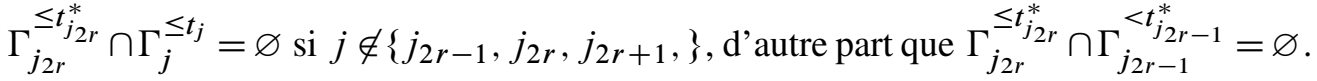

Preuve de (v) Fixons $T=\left(t_{j}\right)_{1 \leq j \leq n} \in \mathcal{T}_{2 r}$. Puisque $j_{2 r}+1 \neq n$ on sait que $j_{2 r}+1 \in \Sigma(T)$ et donc que $t_{2 j}=t_{2 j}^{*}<K$, d'après le Lemme 5.2. Choisissons $\left.t_{j_{2 r}}^{\prime} \in\right] t_{j_{2 r}}^{*}, K\left[\right.$ assez proche de $t_{j_{2 r}}^{*}$ pour que $\left.\Gamma_{j_{2 r}}\right|_{\left.] t_{j_{2 r}}^{*}, t^{\prime} j_{2 r}\right]}$ ne rencontre aucun arc $\Gamma_{j}$ $j \neq j_{2 r}$. La configuration $T^{\prime}$ obtenue à partir de $T$ en remplaçant $t_{j_{2 r}}^{*}$ par $t^{\prime}{ }_{j_{2 r}}$ ne vérifie plus (A) par maximalité de $t_{j_{2 r}}^{*}$. Il existe donc $j \neq j_{2 r}$ tel que $\Gamma_{j_{2 r}}^{t_{2 r}^{*}}$ appartient à un arc $\Gamma_{j}^{\leq t_{j}}, j \neq j_{2 r}$, et ce point, qui est un point simple de $\Gamma_{j}$, n'est pas l'extrémité $\Gamma_{j}^{t_{j}}$. Utilisant le point (iv) qui vient juste d'être prouvé, on en déduit que $j=j_{2 r+1}$ et que (v) est vrai.

Preuve de (iii) et de (vi) Fixons $T=\left(t_{j}\right)_{1 \leq j \leq n} \in \mathcal{T}_{2 r+1}$. On vient de démontrer (v) et on sait donc que $t_{j_{2 r+1}}=t_{j_{2 r+1}}^{*}>-K$. Choisissons $\left.t_{j_{2 r+1}}^{\prime} \in\right]-K, t_{j_{2 r+1}}^{*}[$

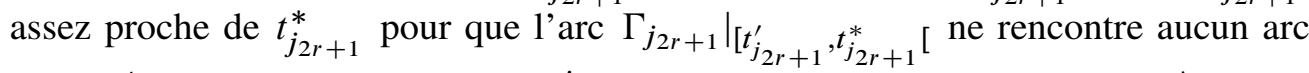
$\Gamma_{j}, j \neq j_{2 r+1}$. La configuration $T^{\prime}$ obtenue à partir de $T$ en remplaçant $t_{j_{2 r+1}}^{*}$ par $t_{j_{2 r+1}}^{\prime}$ n'étant plus dans $\mathcal{T}_{2 r}$, il existe donc $j \neq n$ qui n'est pas dans $\Sigma\left(T^{\prime}\right)$. Il existe donc $j_{2 r+2} \neq j_{2 r+1}$ tel que $\Gamma_{j_{2 r+1}}^{t_{2 r+1}^{*}} \in \Gamma_{j_{2 r+2}}^{\leq t_{j_{2 r+2}}}$. L'entier $j_{2 r+2}$ est le seul entier $j \neq j_{2 r+1}$ tel que $\Gamma_{j_{2 r+1}}^{t_{2 r+1}^{*}} \in \Gamma_{j}$. Nous venons de démontrer (iii) et (vi).

Preuve de l'inégalité $j_{2 r+2}<j_{2 r+1}$ Nous gardons les notations du paragraphe précédent. Utilisant de nouveau le point (v) qui vient juste d'être prouvé, on en déduit que $j_{2 r+1} \in \Sigma\left(T^{\prime}\right)$ et donc que l'entier $j \neq n$ qui n'appartient pas à $\Sigma\left(T^{\prime}\right)$ est $j_{2 r+2}$. En particulier $j_{2 r+2} \neq n$. Puisque $j_{2 r+2} \notin \Sigma\left(T^{\prime}\right), 1$ 'arc $\Gamma_{j_{2 r+2}}^{\leq t_{j_{2}+2}}$ ne rencontre aucun arc autre que lui même, que $\Gamma_{j_{2 r+1}}^{\leq t_{j_{2 r+1}^{*}}^{*}}$ et que $\Gamma_{j_{2 r+2}-1}^{\leq t_{j_{2 r+2}-1}}$. On en déduit que $j_{2 r+2} \neq j_{s}$, si $1 \leq s \leq 2 r+1$. En effet tout arc $\Gamma_{j_{s}}^{\leq t_{j_{s}}}$ rencontre $\Gamma_{j_{s-1}}^{\leq t_{j_{s-1}}}$ et on sait que $j_{s-1} \notin\left\{j_{s}, j_{s}-1, j_{2 r+1}\right\}$, puisque $j_{s-1}>j_{s} \geq j_{2 r+1}$. Il reste à montrer que $j_{2 r+2} \notin\left\{j_{2 s}+1, \ldots, j_{2 s-1}-1\right\}$ si $s \leq r$. Même dans le cas où $s=r$, on sait que $\Gamma_{j_{2 s}}^{t_{j_{2 s}}}=\Gamma_{j_{2 s}}^{t_{j_{2 s}}^{*}}$ appartient à $\Gamma_{j_{2 s+1}}^{<t_{j_{2 s+1}}}=\Gamma_{j_{2 s+1}}^{<t_{j_{2 s+1}}^{*}}$. On sait d'autre part que $\Gamma_{j_{2 s-1}}^{t_{j_{2 s-1}}}=\Gamma_{j_{2 s-1}}^{t_{j_{2 s-1}}^{*}}$ appartient à $\Gamma_{j_{2 s}}^{\leq t_{j_{2 s}}^{*}}$. D'après la Proposition 4.4, on en déduit que 
$\Gamma_{j_{2 s-1}}^{t_{2 s-1}^{*}} \in \Gamma_{j_{2 s}}^{<t_{j_{2 s}}^{*}}$. Ceci implique que $\Gamma_{j_{2 s-1}}^{<t_{j_{2 s-1}}^{*}} \cup \Gamma_{j_{2 s}}^{<t_{j_{2 s}}^{*}}$ est connexe et sépare dans D tout arc $\Gamma_{j^{\prime}}^{\leq-K}, j^{\prime} \in\left\{j_{2 s}+1, \ldots, j_{2 s-1}-1\right\}$, de tout arc $\Gamma_{j^{\prime \prime}}^{\leq-K}, j^{\prime \prime} \notin\left\{j_{2 s}, \ldots, j_{2 s-1}\right\}$. Puisque, par hypothèse, les arcs $\Gamma_{j}^{<t_{j}}$ sont disjoints deux à deux, $\Gamma_{j_{2 s-1}}^{<t_{j_{2 s-1}}^{*}} \cup \Gamma_{j_{2 s}}^{<t_{j_{2}}^{*}}$ sépare $\Gamma_{j^{\prime}}^{<t_{j^{\prime}}}$ de $\Gamma_{j^{\prime \prime}}^{<t_{j^{\prime \prime}}}$. On en déduit que $\Gamma_{j^{\prime}}^{\leq t_{j^{\prime}}} \cap \Gamma_{j^{\prime \prime}}^{\leq t_{j^{\prime \prime}}}=\varnothing$. En effet si ces deux arcs s'intersectaient, ils devraient le faire sur $\Gamma_{j_{2 s-1}}^{<t_{j_{2 s-1}}^{*}} \cup \Gamma_{j_{2 s}}^{<t_{j_{2 s}}^{*}}$, ce qui contredirait la Proposition 4.4. Puisque $j_{2 r+1} \notin\left\{j_{2 s}, \ldots, j_{2 s-1}\right\}$ et puisque $\Gamma_{j_{2 r+1}}^{\leq t_{j_{2 r+1}}} \cap \Gamma_{j_{2 r+2}}^{\leq t_{j_{2 r+2}}} \neq \varnothing$, on en déduit que $j_{2 r+2} \notin\left\{j_{2 s}+1, \ldots, j_{2 s-1}-1\right\}$.

Si $T=\left(t_{i}\right)_{i \in \mathbb{Z} / n \mathbb{Z}} \in \mathcal{T}_{*}$ est une configuration d'ordre $n$, on sait que $\left|t_{i}\right|<K$, pour tout $i \in \mathbb{Z} / n \mathbb{Z}$, d'après le Lemme 5.2. L'ensemble des configurations $T \in \mathcal{T}_{*}$ d'ordre $n$, qui est fermé, est donc compact. Considérons l'ordre lexicographique défini sur $\mathcal{T}$ par un ordre quelquonque sur $\mathbb{Z} / n \mathbb{Z}$. On peut alors trouver une configuration maximale

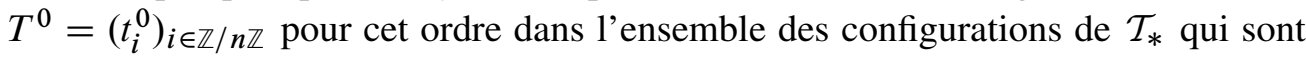
d'ordre $n$. On vérifie facilement, utilisant les arguments vus dans la preuve précédente, que pour tout $i$, il existe $i^{\prime} \neq i$ unique tel que $\Gamma_{i}^{t_{i}^{0}} \in \Gamma_{i^{\prime}}$ et que $\Gamma_{i}^{t_{i}^{0}} \in \Gamma_{i^{\prime}}^{<t_{i^{\prime}}^{0}}$. On se fixera dorénavant une telle configuration et on posera $\Gamma_{i}^{-}=\Gamma_{i}^{\leq t_{i}^{0}}$ et $\sigma_{i}^{0}=\Gamma^{t_{i}^{0}}$. On définira également $\Gamma_{i}^{+}=\Gamma_{i}^{\geq K+1 / 2}$. Sur la Figure 7 nous donnons un exemple avec $n=4$, où l'ordre sur $\mathbb{Z} / 4 \mathbb{Z}$ vérifie $1<2<3<4$. Il n'y a qu'une configuration d'ordre $n=4$ maximale possible dans cet exemple.
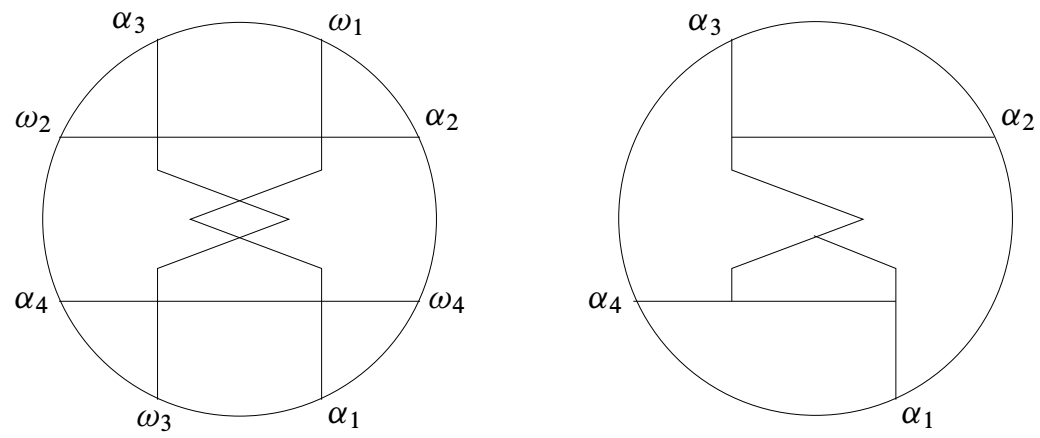

Figure 7

Proposition 5.5 Nous avons les propriétés suivantes:

- $\Gamma_{i}^{+} \cap \Gamma_{i^{\prime}}^{-}=\Gamma_{i}^{+} \cap \Gamma_{i^{\prime}}^{+}=\varnothing$, si $i^{\prime} \neq i$;

- $\Gamma_{i}^{+} \cap \Gamma_{i}^{-}=\varnothing$; 
- $\left(\Gamma_{i}^{-} \backslash\left\{\sigma_{i}^{0}\right\}\right) \cap\left(\Gamma_{i^{\prime}}^{-} \backslash\left\{\sigma_{i^{\prime}}^{0}\right\}\right)=\varnothing$, si $i^{\prime} \neq i$;

- $\Gamma_{i}^{\leq-K} \cap \Gamma_{i^{\prime}}^{-}=\varnothing$, si $i^{\prime} \neq i$;

- l'ensemble $\Gamma_{i}^{-} \backslash \bigcup_{i^{\prime} \neq i} \Gamma_{i^{\prime}}^{-}$est inclus dans une composante connexe de $\mathbf{D} \backslash$ $\bigcup_{i^{\prime} \neq i} \Gamma_{i^{\prime}}^{-}$

- pour tout $i \in \mathbb{Z} / n \mathbb{Z}$, il existe $i^{\prime} \notin\{i, i-1\}$ tel que $\Gamma_{i}^{-} \cap \Gamma_{i^{\prime}}^{-} \neq \varnothing$;

- $s i \Gamma_{i}^{-} \cap \Gamma_{i^{\prime}}^{-} \neq \varnothing$, avec $i^{\prime} \notin\{i, i-1\}$, alors la composante connexe de $\mathbf{D} \backslash\left(\Gamma_{i}^{-} \cup \Gamma_{i^{\prime}}\right)$ qui contient $\Gamma_{i-1}^{\leq-K}$ est différente de celle qui contient $\Gamma_{i-1}^{+}$.

Démonstration Le seul point qui n'est pas immédiat ou qui n'a pas été mentionné précédemment est le cinquième point. Il n'est cependant pas très difficile à prouver. $\mathrm{Si}$ le point $\Gamma_{i}^{t}, t<t_{i}^{0}$, n'appartient à aucun $\Gamma_{i^{\prime}}^{-}, i^{\prime} \neq i$, il n'y a en effet aucune difficulté à perturber l'arc $\Gamma_{i}$ sur $[-K, t]$ pour qu'il aboutisse en $\Gamma_{i}^{t}$ tout en évitant $\bigcup_{i^{\prime} \neq i} \Gamma_{i^{\prime}}^{-}$. En effet on peut contourner chaque point de $\left.\Gamma_{i}\right|_{[-K, t]}$ qui est sur un $\Gamma_{i^{\prime}}^{-}, i^{\prime} \neq i$, au voisinage de ce point (voir Figure 8).

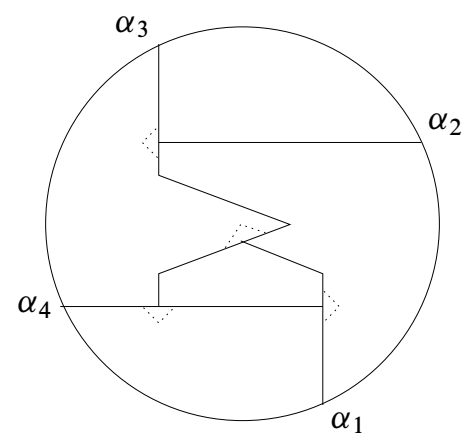

Figure 8

\section{Construction du graphe modèle}

Nous allons construire dans cette section un graphe $G$ qui sera le modèle unidimensionnel de notre décomposition en briques finale. Rappelons que l'extrémité $\sigma_{i}^{0}$ de $\Gamma_{i}^{-}$ appartient à un unique arc $\Gamma_{i^{\prime}}^{-}, i^{\prime} \neq i$, que c'est un point simple de $\Gamma_{i}$ et de $\Gamma_{i^{\prime}}$, qu'il n'appartient pas à $O_{i}$ et que $\sigma_{i}^{0} \neq \sigma_{i^{\prime}}^{0}$. On appelera singularité de $\Gamma_{i}^{-}$tout point qui appartient à $O_{i}$ ou qui est l'extrémité d'un arc $\Gamma_{i^{\prime}}^{-}$. Les singularités étant des points simples de $\Gamma_{i}$, on peut les paramétrer sous la forme $\sigma_{i}^{l}=\Gamma_{i}\left(t_{i}^{l}\right)$, où $\left(t_{i}^{l}\right)_{l \leq 0}$ est une suite strictement croissante uniquement déterminée. On se fixe alors $L$ suffisament 
grand pour que $t_{i}^{-L} \leq-K$. Les singularités $\sigma_{i}^{l}, l \leq-L$, sont alors nécessairement des points de l'orbite $O_{i}$. Remarquons qu'on ne peut pas trouver $i_{0}$ et $i_{1}$ tels que

$$
\sigma_{i_{0}}^{-1}=\sigma_{i_{1}}^{0}, \quad \sigma_{i_{0}}^{0}=\sigma_{i_{1}}^{-1} \text {. }
$$

En effet, supposons que $i_{0}>i_{1}$ (pour l'ordre sur $\mathbb{Z} / n \mathbb{Z}$ qui nous a permi de définir $\left.T^{0}\right)$. Si $t>t_{i}^{0}$ est proche de $t_{i}^{0}$, la configuration $T^{\prime}=\left(t_{i}\right)_{i \in \mathbb{Z} / n \mathbb{Z}}$ où $t_{i_{0}}^{\prime}=t, t_{i_{1}}^{\prime}=t_{i_{1}}^{-1}$ et $t_{i}^{\prime}=t_{i}^{0}$ si $i \notin\left\{i_{0}, i_{1}\right\}$ vérifie les conditions (A) et (B) et est plus grande que $T^{0}$ pour l'ordre lexicographique, ce qui contredit la maximalité de $T^{0}$.

Définissons maintenant une suite $\left(t^{l}\right)_{l \geq 1}$ en posant

$$
\left\{\begin{array}{l}
t^{1}=K+1 / 2 \\
t^{l}=K+l-1 \quad \text { si } l \geq 2
\end{array}\right.
$$

et définissons les singularités $\sigma_{i}^{l}=\Gamma_{i}\left(t^{l}\right)$ de $\Gamma_{i}^{+}$. La Proposition 3.1 nous permet d'affirmer l'existence d'une suite de disques de sécurité $\left(V_{i}^{l}\right)_{l \neq 0}$ adaptée à notre nouvelle indexation. Plus précisément:

Lemme 6.1 On peut construire, pour tout $i \in \mathbb{Z} / n \mathbb{Z}$, une suite $\left(V_{i}^{l}\right)_{l \neq 0}$ de disques ouverts telle que:

- chaque $V_{i}^{l}$ contient le segment $\left.\Gamma_{i}\right|_{\left[t_{i}^{l}, t_{i}^{l+1}\right]}$;

- $V_{i}^{l} \cap V_{i^{\prime}}^{l^{\prime}}=\varnothing$, si $i \neq i^{\prime}$ et $l>0$;

- $V_{i}^{l} \cap V_{i}^{l^{\prime}}=\varnothing$, si $l^{\prime}<0<l$;

- $f\left(V_{i}^{l}\right) \cap V_{i}^{l^{\prime}}=\varnothing$, si $l^{\prime}<l$;

- la suite $\left(\bar{V}_{i}^{l}\right)_{l>0}$ converge vers $\left\{\omega_{i}\right\}$;

- la suite $\left(\bar{V}_{i}^{l}\right)_{l<0}$ converge vers $\left\{\alpha_{i}\right\}$.

On définit maintenant deux suites d'arcs d'attache $\left(\alpha_{i}^{l}\right)_{l<0}$ et $\left(\alpha_{i}^{l}\right)_{l>0}$ de la façon suivante:

$\alpha_{i}^{-1}=\left.\Gamma_{i}\right|_{\left[t_{i}^{-1}, t_{i}^{0}\right]}$

$\alpha_{i}^{1}=\left.\Gamma_{i}\right|_{\left[t_{i}^{1}, t_{i}^{2}\right]}$

si $l<-1$, alors $\alpha_{i}^{l}=\left.\Gamma_{i}\right|_{\left[t_{i}^{l}, t_{i}^{\prime l}\right]}$, en notant ${t^{\prime}}_{i}^{l}$ le premier instant où $\left.\Gamma_{i}\right|_{\left[t_{i}^{l}, t_{i}^{l+1}\right]}$ rencontre $\bigcup_{l<m<0} \alpha_{i}^{m}$ 
si $l>1$, alors $\alpha_{i}^{l}=\left.\Gamma_{i}\right|_{\left[t_{i}^{\prime}, t_{i}^{l+1}\right]}$, en notant $t_{i}^{\prime l}$ le dernier instant où $\left.\Gamma_{i}\right|_{\left[t_{i}^{l}, t_{i}^{l+1}\right]}$ rencontre $\bigcup_{0<m<l} \alpha_{i}^{m}$.

Remarquons que $\alpha_{i}^{l}$ est un segment (i.e. ne se réduit pas à un point) puisque les singularités sont des points simples de $\Gamma_{i}$. Remarquons également que $\alpha_{i}^{l} \subset V_{i}^{l}$. Toujours grâce à la Proposition 4.4, on sait que pour tout $l<-1$, le point $\Gamma_{i}\left(t^{\prime l}{ }_{i}\right)$ appartient à un unique arc $\alpha_{i}^{m}, l<m<0$; qu'il est différent de l'extrémité $\Gamma_{i}\left(t_{i}^{\prime m}\right)$ et qu'il ne peut être égal à l'extrémité $\sigma_{i}^{m}$ que dans le cas où $m=l-1$ et $t_{i}^{\prime l}=t_{i}^{l-1}$. Nous avons un résultat analogue pour les $\alpha_{i}^{l}, l>0$. Parmi les singularités, nous porterons particulièrement attention à la singularité $\sigma_{i}^{*}=\Gamma_{i}\left[\left(t_{i}^{0}\right)\right]$, où $[t]$ désigne la partie entière d'un réel $t$. Il s'agit de la singularité de $\Gamma_{i}^{-}$la plus "proche" de l'extrémité $\sigma_{i}^{0}$ parmi les singularités qui sont sur $O_{i}$. Si on écrit $\sigma_{i}^{*}=\sigma_{i}^{l_{i}}$, on peut remarquer que $\alpha_{i}^{l}=\left.\Gamma_{i}\right|_{\left[t_{i}^{l}, t_{i}^{l+1}\right]}$ si $l_{i} \leq l<0$

On construit ainsi des arbres localement finis $A_{i}^{-}=\bigcup_{l<0} \alpha_{i}^{l}$ et $A_{i}^{+}=\bigcup_{l>0} \alpha_{i}^{l}$ : seul un nombre fini de $\alpha_{i}^{l}$ rencontrent une partie compacte de $\mathbf{D}$ donnée. L'arbre $A_{i}^{-}$est inclus dans $\Gamma_{i}^{-}$et contient toutes les singularités de $\Gamma_{i}^{-}$. C'est sur le graphe $G=\bigcup_{i \in \mathbb{Z} / n \mathbb{Z}}\left(A_{i}^{-} \cup A_{i}^{+}\right)$que nous allons modeler notre décomposition. Nous illustrons la construction du graphe $G$ sur la Figure 9. Les singularités qui correspondent à des points de l'orbite sont représentées par des points noirs, celles qui sont des extrémités sont représentées par des points blancs.

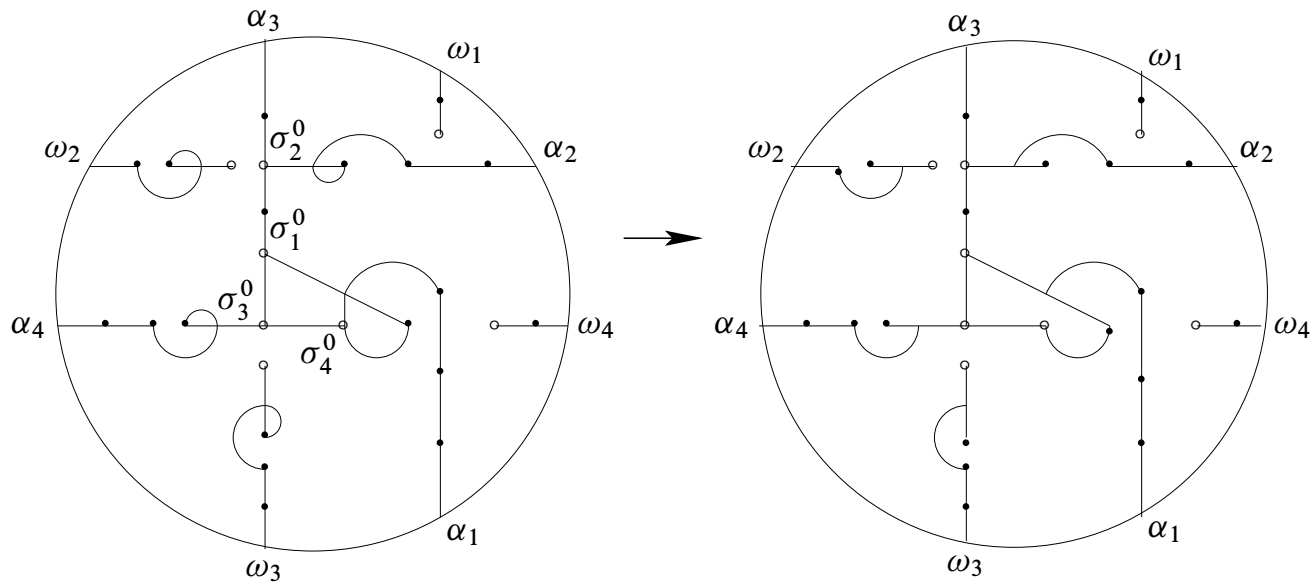

Figure 9

On déduit facilement de la propriété 5.5 et des inclusions $A_{i}^{-} \subset \Gamma_{i}^{-}$et $A_{i}^{+} \subset \Gamma_{i}^{+}$le résultat qui suit: 
Proposition 6.2 Nous avons les propriétés suivantes:

- $A_{i}^{+} \cap A_{i^{\prime}}^{-}=\varnothing$, pour tous $i^{\prime}$ et $i$ dans $\mathbb{Z} / n \mathbb{Z}$;

- $A_{i}^{+} \cap A_{i^{\prime}}^{+}=\varnothing$, si $i \neq i^{\prime}$;

- $\left(A_{i}^{-} \backslash\left\{\sigma_{i}^{0}\right\}\right) \cap\left(A_{i^{\prime}}^{-} \backslash\left\{\sigma_{i^{\prime}}^{0}\right\}\right)=\varnothing$, si $i \neq i^{\prime}$;

- $\alpha_{i}^{l} \cap A_{i^{\prime}}=\varnothing$, si $i \neq i^{\prime}$ et $l<-L$;

- l'ensemble $A_{i}^{-} \backslash \bigcup_{i^{\prime} \neq i} A_{i^{\prime}}^{-}$est inclus dans une composante connexe de $\mathbf{D} \backslash \bigcup_{i^{\prime} \neq i} A_{i^{\prime}}^{-}$;

- pour tout $i \in \mathbb{Z} / n \mathbb{Z}$, il existe $i^{\prime} \notin\{i, i-1\}$ tel que $A_{i}^{-} \cap A_{i^{\prime}}^{-} \neq \varnothing$;

- si $A_{i}^{-} \cap A_{i^{\prime}}^{-} \neq \varnothing$, avec $i^{\prime} \notin\{i, i-1\}$, alors la composante connexe de $\mathbf{D} \backslash\left(A_{i}^{-} \cup A_{i^{\prime}}^{-}\right)$qui contient les $\alpha_{i-1}^{l}, l<-L$, est différente de celle qui contient $A_{i-1}^{+}$.

\section{Construction d'une décomposition adaptée}

Nous construirons dans cette section une décomposition en briques libre maximale modelée sur le graphe $G=\bigcup_{i \in \mathbb{Z} / n \mathbb{Z}}\left(A_{i}^{-} \cup A_{i}^{+}\right)$. Nous utiliserons implicitement le théorème de Schoenflies, ou plutôt la version généralisée qu'en donne Homma [13]. La construction se fera en plusieurs étapes. Commençons par la plus simple, en rappelant

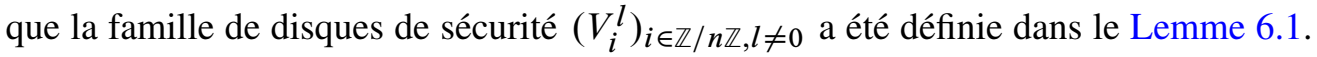

Construction de la famille $\left(b^{\prime l}\right)_{i \in \mathbb{Z} / n \mathbb{Z}, l>0}$

Lemme 7.1 On peut construire une famille $\left(b_{i}^{\prime l}\right)_{i \in \mathbb{Z} / n \mathbb{Z}, l>0}$ de disques fermés de $\mathbf{D}$ telle que:

(i) chaque $b_{i}^{\prime l}$ est libre et inclus dans $V_{i}^{l}$;

(ii) $\operatorname{Int}\left({b^{\prime}}_{i}^{\prime l}\right) \cap \operatorname{Int}\left({b^{\prime}}_{i}^{l^{\prime}}\right)=\varnothing$, si $l \neq l^{\prime}$;

(iii) $\operatorname{Int}\left(b_{i}^{\prime 1}\right)$ contient $\sigma_{i}^{1}$ et $\sigma_{i}^{2}$;

(iv) $\operatorname{Int}\left(b_{i}^{\prime l}\right)$ contient $\sigma_{i}^{l+1}$, si $l \geq 2$;

(v) $\operatorname{Int}\left(\bigcup_{m \leq l} b_{i}^{\prime l}\right)$ contient $\bigcup_{m \leq l} \alpha_{i}^{l}$;

(vi) $\alpha_{i}^{l}, l>1$, rencontre un unique disque $b_{i}^{\prime m}, m<l$, que l'on note ${b^{\prime}}_{i}^{m[l]}$;

(vii) si $b_{i}^{\prime m}$ intersecte un arc $\alpha_{i}^{l}=\left.\Gamma_{i}\right|_{\left[t_{i}^{\prime \prime}, t_{i}^{l+1}\right]}, l>m$, alors $b_{i}^{\prime m} \cap \alpha_{i}^{l}$ est un soussegment non trivial $\left.\Gamma_{i}\right|_{\left[t_{i}^{\prime}, t_{i}^{\prime \prime l}\right]}$ de $\alpha_{i}^{l}$ et $\left.\Gamma_{i}\right|_{\left[t_{i}^{\prime}{ }_{i}, t^{\prime \prime}{ }_{i}[\right.} \subset \operatorname{Int}\left(b_{i}^{\prime m}\right)$; 
(viii) un disque $b_{i}^{\prime l}, l>1$, ne rencontre aucun disque $b_{i}^{\prime m}, m<l$, autre que ${b^{\prime}}_{i}^{m[l]}$;

(ix) $b_{i}^{\prime l} \cap{b^{\prime}}_{i}^{m[l]}=\partial{b^{\prime}}_{i}^{l} \cap \partial{b^{\prime}}_{i}^{m[l]}$ est un segment.

Démonstration Puisque $\left.\Gamma_{i}\right|_{[K, K+1]}$ est un arc de translation, le segment $\alpha_{i}^{1}=$ $\left.\Gamma_{i}\right|_{[K+1 / 2, K+1]}$ est libre. Nous pouvons donc construire un disque fermé $b_{i}^{\prime 1} \subset V_{i}^{1}$ qui est libre et dont l'intérieur contient $\alpha_{i}^{1}$. Nous pouvons de plus supposer que $b_{i}^{\prime 1}$ ne contient aucune singularité $\sigma_{i}^{l}, l>2$. Soyons plus précis. Il n'y a qu'un nombre fini d'arcs $\alpha_{i}^{l}, l>1$, qui rencontrent $\alpha_{i}^{1}$. Nous pouvons supposer, d'une part que ce sont les seuls que rencontre $b_{i}^{\prime}{ }_{i}$, d'autre part que $b_{i}^{\prime}$ vérifie la condition (vii) du Lemme 7.1 .

Supposons maintenant que les disques $b_{i}^{\prime m}$ ont été construits jusqu'à l'ordre $l$ et qu'ils vérifient les conditions du Lemme 7.1. Ceci a un sens si on remplace la condition (vi) par la condition suivante:

(vi') un arc d'attache $\alpha_{i}^{l^{\prime}}, l^{\prime}>1$, rencontre au plus un disque $b_{i}^{\prime m}, m \leq \min \left(l, l^{\prime}-1\right)$. Considérons l'arc d'attache $\alpha_{i}^{l+1}=\left.\Gamma_{i}\right|_{\left[t^{\prime \prime}{ }^{l+1}, t_{i}^{l+2}\right]}$. Nous savons que $\Gamma_{i}\left(t^{\prime l+1}\right)$ appartient à un arc $\alpha_{i}^{m}, m \leq l$, et donc à un disque $b_{i}^{m^{\prime}}, m^{\prime} \leq l$ d'après (v). Ce disque est unique d'après (vi'), on le note $b_{i}^{m[l+1]}$. D'après (viii), nous savons que $\alpha_{i}^{l+1} \cap b_{i}^{m[l+1]}=\left.\Gamma_{i}\right|_{\left[t^{\prime}{ }_{i}+1, t^{\prime \prime}{ }_{i}^{l+1}\right]}$, où $t_{i}^{\prime l+1}<t^{\prime \prime l}{ }_{i}^{l+1}<t_{i}^{l+2}$. Le segment $\left.\Gamma_{i}\right|_{\left[t^{\prime \prime}{ }_{i}+1, t_{i}^{l+2}\right]}$ est libre, puisque $\left.\Gamma_{i}\right|_{\left[t_{i}^{l+1}, t_{i}^{l+2}\right]}$ est un arc de translation. Il est également inclus dans $V_{i}^{l+1}$. On peut donc construire un disque fermé $b_{i}^{\prime l+1} \subset V_{i}^{l+1}$ qui est libre, dont l'intérieur contient $\left.\Gamma_{i}\right|_{\left.] t^{\prime \prime \prime}{ }_{i}+1, t_{i}^{l+2}\right]}$, et qui ne rencontre qu'un seul disque ${b^{\prime}}_{i}^{m}, m \leq l$, à savoir ${b^{\prime}}_{i}^{m[l+1]}$. On peut également supposer que ${b^{\prime}}_{i}^{l+1} \cap{b^{\prime}}_{i}^{m[l+1]}=\partial{b^{\prime}}_{i}^{l+1} \cap \partial{b^{\prime}}_{i}^{m[l+1]}$ est un segment dont $\Gamma_{i}\left(t_{i}^{\prime \prime l}{ }_{i}+1\right)$ n'est pas une extrémité. Tout arc d'attache $\alpha_{i}^{l^{\prime}}, l^{\prime}>l+1$, qui rencontre $\left.\Gamma_{i}\right|_{\left[t_{i}^{\prime \prime}{ }_{i}+t_{i}+2\right]}$ est disjoint de tous les disques $b_{i}^{\prime m}, m \leq l$, d'après (vii). On peut donc supposer que $b_{i}^{\prime l+1}$ vérifie également la condition (vii). Remarquons maintenant que la famille $\left(b_{i}^{\prime m}\right)_{1 \leq m \leq l+1}$ vérifie toutes les conditions du lemme. Nous avons dessiné les disques $b_{i}^{\prime l}, i \in \mathbb{Z} / n \mathbb{Z}, l>0$, sur la Figure 10.

\section{Construction de la famille $\left(b_{i}^{l}\right)_{i \in \mathbb{Z} / n \mathbb{Z}, l<0}$}

La construction sera similaire, à savoir un épaississement des arbres $A_{i}^{-}, i \in \mathbb{Z} / n \mathbb{Z}$. La situation est cependant plus délicate car ces arbres ne sont pas disjoints. Rappelons que l'extrémité $\sigma_{i}^{0}$ n'est pas sur $O_{i}$ et que le segment $\left.\Gamma_{i}\right|_{\left[\left[t_{i}^{0}\right], t_{i}^{0}\right]}$ qui joint la singularité $\sigma_{i}^{*}$ à $\sigma_{i}^{0}$ est donc libre. Il en est de même, bien sûr, du sous-segment $\alpha_{i}^{-1}$. Rappelons également que $\sigma_{i}^{0}$, qui appartient à un unique arc $\Gamma_{i_{0}}, i_{0} \neq i$, est sur $A_{i_{0}}^{-}$. Dans le 


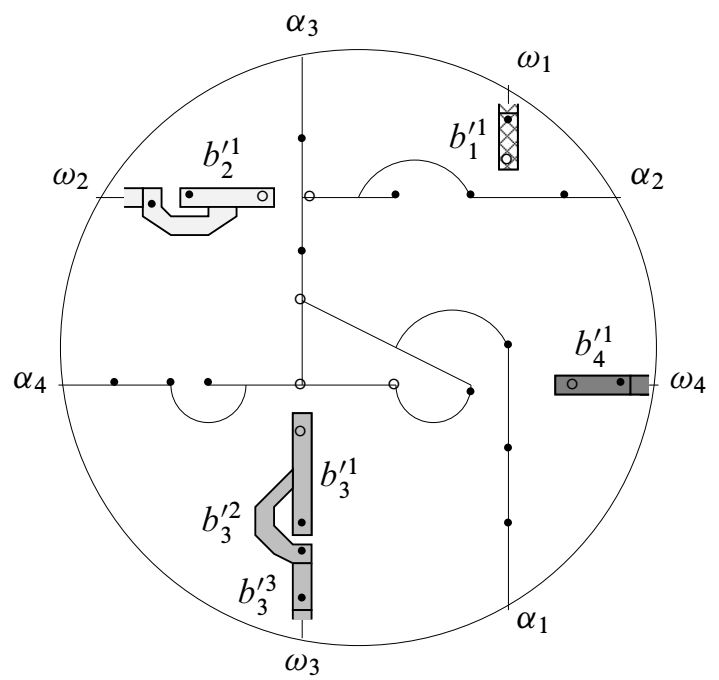

Figure 10

cas où $\sigma_{i}^{-1}=\sigma_{i}^{*}$, on sait que $\alpha_{i}^{-1} \backslash \sigma_{i}^{0}$ est disjoint de tout arc $\Gamma_{i^{\prime}}^{-}, i^{\prime} \neq i$, et donc de $A_{i^{\prime}}^{-}$. Dans le cas contraire, $\sigma_{i}^{-1}$ appartient à un unique $\operatorname{arc} \Gamma_{i_{1}}, i_{1} \neq i$, et c'est l'extrémité $\sigma_{i_{1}}^{0}$ de $A_{i_{1}}^{-}$. Dans ce cas $\operatorname{int}\left(\alpha_{i}^{1}\right)$ ne rencontre aucun arc $\Gamma_{i^{\prime}}^{-}, i^{\prime} \neq i$. On peut donc construire une famille $\left(b_{i}^{-1}\right)_{i \in \mathbb{Z} / n \mathbb{Z}}$ de disques fermés libres de $\mathbf{D}$, vérifiant $\alpha_{i}^{-1} \subset b_{i}^{-1} \subset V_{i}^{-1}$, dont les intérieurs sont disjoints deux à deux, et tels que

$$
b_{i}^{-1} \cap b_{i^{\prime}}^{-1} \neq \varnothing \text { et } i \neq i^{\prime} \Longrightarrow \sigma_{i}^{0}=\sigma_{i^{\prime}}^{-1} \text { ou } \sigma_{i}^{-1}=\sigma_{i^{\prime}}^{0}
$$

On va être plus précis. Pour toute singularité $\sigma_{i}^{l}=\Gamma_{i}\left(t_{i}^{l}\right), l<0$, choisissons $\varepsilon_{i}^{l}>0$ tel que:

- $t_{i}^{l}+\varepsilon_{i}^{l}<t_{i}^{\prime l}$

- $\left.\Gamma_{i}\right|_{\left[t_{i}^{l}-\varepsilon_{i}^{l}, t_{i}^{l}+\varepsilon_{i}^{l}\right]} \subset V_{i}^{l}$;

- $\left.\quad \Gamma_{i}\right|_{\left[t_{i}^{l}-\varepsilon_{i}^{l}, t_{i}^{l}+\varepsilon_{i}^{l}\right]} \cap\left(\bigcup_{i^{\prime} \neq i} \Gamma_{i^{\prime}}\right)=\varnothing$, si $\sigma_{i}^{l} \in O_{i}$;

- $\left.\Gamma_{i}\right|_{\left[t_{i}^{l}-\varepsilon_{i}^{l}, t_{i}^{l}+\varepsilon_{i}^{l}\right]} \cap\left(\bigcup_{i^{\prime} \neq i} \Gamma_{i^{\prime}}\right)=\left\{\sigma_{i}^{l}\right\}$, si $\sigma_{i}^{l} \notin O_{i}$.

Lemme 7.2 On peut constuire une famille $\left(b_{i}^{-1}\right)_{i \in \mathbb{Z} / n \mathbb{Z}}$ de disques fermés de $\mathbf{D}$ et une famille $\left(\eta_{i}^{-1}\right)_{i \in \mathbb{Z} / n \mathbb{Z}}$ de réels $>0$, telles que:

(i) $b_{i}^{-1}$ contient $\alpha_{i}^{-1}$;

(ii) $b_{i}^{-1}$ est libre et inclus dans $V_{i}^{-1}$;

(iii) $\operatorname{Int}\left(b_{i}^{-1}\right) \cap \operatorname{Int}\left(b_{i^{\prime}}^{-1}\right)=\varnothing$, si $i \neq i^{\prime}$; 
(iv) si $b_{i}^{-1}$ intersecte un arc d'attache $\alpha_{i}^{l}=\left.\Gamma_{i}\right|_{\left[t_{i}^{l}, t^{\prime}{ }_{i}\right]}, l \leq-2$, alors $b_{i}^{1} \cap \alpha_{i}^{l}$ est un sous-segment $\left.\Gamma_{i}\right|_{\left[t^{\prime \prime}{ }_{i}, t^{\prime}{ }_{i}\right]}$ de $\alpha_{i}^{l}$, avec $t_{i}^{l}<t^{\prime \prime l}{ }_{i}^{l}<t^{\prime l}{ }_{i}$;

(v) $0<\eta_{i}^{-1}<\min \left(\varepsilon_{i}^{-1}, \varepsilon_{i_{0}}^{l_{0}}\right)$, où on écrit $\sigma_{i}^{0}=\sigma_{i_{0}}^{l_{0}}$;

(vi) $b_{i}^{-1} \cap A_{i_{0}}^{-}=\left.\Gamma_{i_{0}}\right|_{\left[t_{i_{0}}^{l_{0}}, t_{i}^{l_{0}}+\eta_{i}^{-1}\right]}$;

(vii) si $\sigma_{i}^{-1}=\sigma_{i}^{*}$, alors $b_{i}^{-1} \cap A_{i^{\prime}}^{-}=b_{i}^{-1} \cap b_{i^{\prime}}^{-1}=\varnothing$, si $i^{\prime} \notin\left\{i, i_{0}\right\}$;

(viii) si $\sigma_{i}^{-1}=\sigma_{i}^{*}$, alors $\sigma_{i}^{-1} \in \operatorname{Int}\left(b_{i}^{-1}\right)$;

(ix) si $\sigma_{i}^{-1}=\sigma_{i_{1}}^{0}$ est une extrémité, alors $b_{i}^{-1} \cap A_{i^{\prime}}^{-}=b_{i}^{-1} \cap b_{i^{\prime}}^{-1}=\varnothing$, si $i^{\prime} \notin$ $\left\{i, i_{0}, i_{1}\right\}$;

(x) si $\sigma_{i}^{-1}=\sigma_{i_{1}}^{0}$ est une extrémité, alors $\partial b_{i}^{-1}$ contient $\left.\Gamma_{i}\right|_{\left[t_{i}^{-1}-\eta_{i}^{-1}, t_{i}^{-1}+\eta_{i}^{-1}\right]}$;

(xi) si $\sigma_{i}^{-1}=\sigma_{i_{1}}^{0}$ est une extrémité, alors

$$
\left.b_{i}^{-1} \cap b_{i_{1}}^{-1}=\partial b_{i}^{-1} \cap \partial b_{i_{1}}^{-1}=\left.\Gamma_{i_{1}}\right|_{\left[t_{i_{1}}, t_{i_{1}}\right.} ^{l_{1}}, \min \left(\eta_{i}^{-1}, \eta_{i_{1}}^{-1}\right)\right]^{l_{1}}
$$

Nous avons dessiné les disques $b_{i}^{-1}, i \in \mathbb{Z} / n \mathbb{Z}$, sur la Figure 11 .

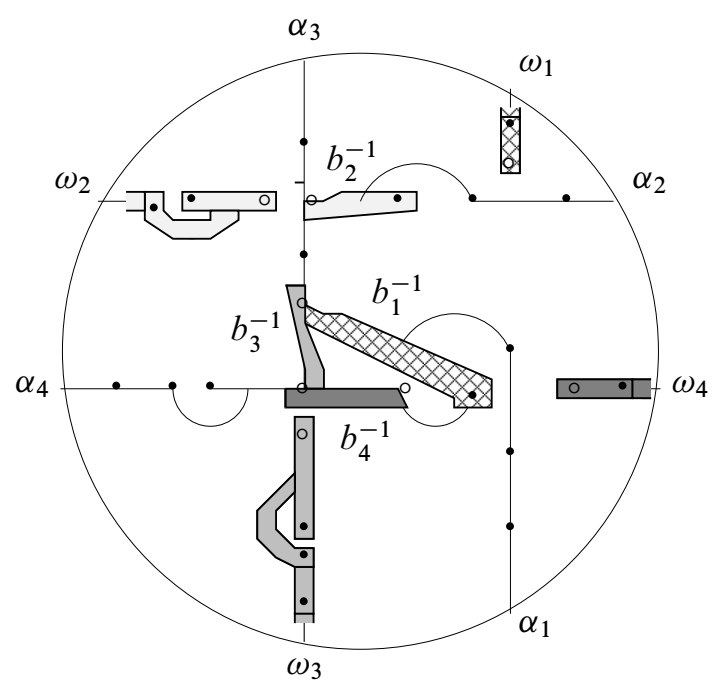

Figure 11

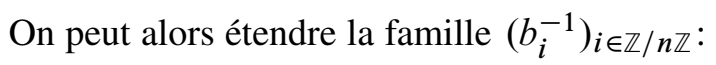

Lemme 7.3 On peut étendre la famille $\left(b_{i}^{-1}\right)_{i \in \mathbb{Z} / n \mathbb{Z}}$ en une famille $\left(b_{i}^{l}\right)_{i \in \mathbb{Z} / n \mathbb{Z}, l<0}$ de disques fermés de $\mathbf{D}$ et la famille $\left(\eta_{i}^{-1}\right)_{i \in \mathbb{Z} / n \mathbb{Z}}$ en une famille $\left(\eta_{i}^{l}\right)_{i \in \mathbb{Z} / n \mathbb{Z}, l<0}$ de réels $>0$ telles que: 
(i) $0<\eta_{i}^{l}<\varepsilon_{i}^{l}$;

(ii) chaque $b_{i}^{l}$ est libre et inclus dans $V_{i}^{l}$;

(iii) $\sigma_{i}^{l} \in \operatorname{Int}\left(b_{i}^{l}\right)$, si $\sigma_{i}^{l} \in O_{i}$;

(iv) $\sigma_{i}^{l} \in \partial b_{i}^{l}$, si $\sigma_{i}^{l} \notin O_{i}$, plus précisément, $\left.\Gamma_{i}\right|_{\left[t_{i}^{l}-\eta_{i}^{l}, t_{i}^{l}+\eta_{i}^{l}\right]} \subset \partial b_{i}^{l}$;

(v) $\bigcup_{m \geq l} b_{i}^{m}$ contient $\bigcup_{m \geq l} \alpha_{i}^{m}$;

(vi) $\operatorname{Int}\left(b_{i}^{l}\right) \cap \operatorname{Int}\left(b_{i^{\prime}}^{l^{\prime}}\right)=\varnothing$, si $(i, l) \neq\left(i^{\prime}, l^{\prime}\right)$;

(vii) $\operatorname{Int}\left(b_{i}^{l}\right) \cap A_{i^{\prime}}^{-}=\varnothing$, si $i \neq i^{\prime}$;

(viii) un arc d'attache $\alpha_{i}^{l}, l<-1$, rencontre un unique disque $b_{i}^{m}, m>l$, que l'on note $b_{i}^{m[l]}$

(ix) si $b_{i}^{m}$ intersecte un arc d'attache $\alpha_{i}^{l}=\left.\Gamma_{i}\right|_{\left[t_{i}^{l}, t_{i}^{\prime \prime}\right]}, l<m$, alors $b_{i}^{m} \cap \alpha_{i}^{l}$ est un sous-segment $\left.\Gamma_{i}\right|_{\left[t^{\prime \prime}{ }_{i}, t^{\prime \prime}{ }_{i}+1\right]}$ de $\alpha_{i}^{l}$, où $t_{i}^{l}<t^{\prime \prime l}{ }_{i}<t_{i}^{l}$;

(x) un disque $b_{i}^{l}, l<-1$, ne rencontre aucun disque $b_{i}^{m}, m>l$, autre que $b_{i}^{m[l]}$;

(xi) $b_{i}^{l} \cap b_{i}^{m[l]}=\partial b_{i}^{l} \cap \partial b_{i}^{m[l]}$ est un segment;

(xii) un disque $b_{i}^{l}, l<-1$, ne rencontre aucun disque $b_{i^{\prime}}^{l^{\prime}}, i^{\prime} \neq i$, si $\sigma_{i}^{l} \in O_{i}$;

(xiii) un disque $b_{i}^{l}, l<-1$, ne rencontre aucun disque $b_{i^{\prime}}^{l^{\prime}}, i^{\prime} \neq i$, autre que $b_{i_{1}}^{-1}$, si $\sigma_{i}^{l}$ est l'extrémité $\sigma_{i_{1}}^{0}$, et on a $b_{i}^{l} \cap b_{i_{1}}^{-1}=\partial b_{i}^{l} \cap \partial b_{i_{1}}^{-1}=\left.\Gamma_{i}\right|_{\left[t_{i}^{l}, t_{i}^{l}+\eta_{i_{1}}^{-1}\right]}$.

Démonstration On raisonne par récurrence, comme dans la preuve du Lemme 7.1. On suppose donc qu'une famille $\left(b_{i}^{m}\right)_{i \in \mathbb{Z} / n \mathbb{Z}, m \geq l}$ a été construite qui vérifie toutes les hypothèses du Lemme 7.3 en remplaçant (viii) par:

(viii') un arc d'attache $\alpha_{i}^{l^{\prime}}, l^{\prime}<0$, rencontre au plus un disque $b_{i}^{m}, m \geq \max \left(l, l^{\prime}+1\right)$.

Fixons $i \in \mathbb{Z} / n \mathbb{Z}$ et considérons l'arc d'attache $\alpha_{i}^{l-1}=\left.\Gamma_{i}\right|_{\left[t_{i}^{l-1}, t_{i}^{\prime l}\right]}$.

Nous savons que $\Gamma_{i}\left(t_{i}^{\prime l-1}\right)$ appartient à un arc $\alpha_{i}^{m}, m \geq l$, et donc à un disque $b_{i}^{m^{\prime}}$, $m^{\prime} \geq l$, d'après (v). D'après (viii'), ce disque est le seul disque $b_{i}^{m}, m \geq l$ que rencontre $\alpha_{i}^{l-1}$. On le note $b_{i}^{m[l-1]}$. D'après (ix), nous savons que $\alpha_{i}^{l-1} \cap b_{i}^{m[l-1]}=$ $\left.\Gamma_{i}\right|_{\left[t_{i}^{\prime \prime l}{ }_{i}, t_{i}^{\prime l-1}\right]}$, où $t_{i}^{l-1}<t_{i}^{\prime \prime l} l-1<t_{i}^{\prime l-1}$. Le segment $\left.\Gamma_{i}\right|_{\left.{ }_{t_{i}}^{l-1}, t^{\prime \prime \prime}{ }_{i}-1\right]}$ est libre et inclus dans $V_{i}^{l-1}$. Dans le cas où $\sigma_{i}^{l-1} \in O_{i}$, le segment $\left.\Gamma_{i}\right|_{\left[t_{i}^{l-1}, t_{i}^{\prime \prime l}{ }_{i}-1\right.}$ ne rencontre aucun arbre $A_{i^{\prime}}^{-}, i^{\prime} \neq i$, et aucun disque $b_{i^{\prime}}^{m}, i^{\prime} \neq i, m \geq l$. Dans le cas où $\sigma_{i}^{l-1}$ est l'extrémité $\sigma_{i_{1}}^{0}$, ce segment ne rencontre aucun arbre $A_{i^{\prime}}^{-}, i^{\prime} \notin\left\{i, i_{1}\right\}$, et n'intersecte $A_{i_{1}}^{-}$qu'en son extrémité $\sigma_{i_{1}}^{0}$. De même, il ne rencontre aucun disque $b_{i^{\prime}}^{m}, i^{\prime} \neq i$, 
$m \geq l$, autre que $b_{i_{1}}^{-1}$ et on a $\left.b_{i_{1}}^{-1} \cap \Gamma_{i}\right|_{\left[t_{i}^{l-1}, t_{i}^{\prime \prime l}{ }_{i}\right]}=\left.\Gamma_{i}\right|_{\left[t_{i}^{l-1}, t_{i}^{l-1}+\eta_{i_{1}}^{-1}\right]}$. On peut donc

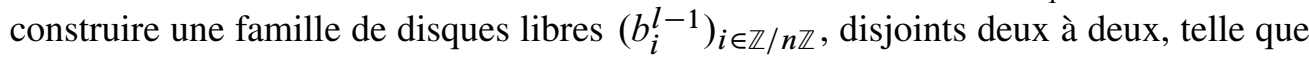

- $b_{i}^{l-1}$ est inclus dans $V_{i}^{l-1}$,

- $b_{i}^{l-1}$ contient $\left.\Gamma_{i}\right|_{\left[t_{i}^{l-1}, t^{\prime \prime}{ }_{i}^{l-1}\right]}$;

- $b_{i}^{l-1}$ ne rencontre aucun disque $b_{i}^{m}, m \geq l$, autre que $b_{i}^{m[l-1]}$;

- $b_{i}^{l-1} \cap b_{i}^{m[l-1]}=\partial b_{i}^{l-1} \cap \partial b_{i}^{m[l-1]}$ est un segment dont $\Gamma\left(t_{i}^{\prime \prime l-1}\right)$ n'est pas une extrémité;

- $b_{i}^{l-1}$ ne rencontre aucun disque $b_{i^{\prime}}^{m^{\prime}}, i^{\prime} \neq i, m^{\prime} \geq l$, dans le cas où $\sigma_{i}^{l-1} \in O_{i}$;

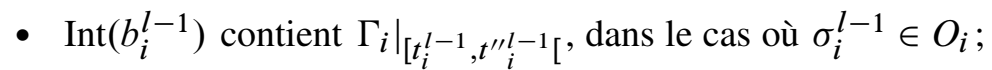

- $b_{i}^{l-1}$ ne rencontre aucun disque $b_{i^{\prime}}^{m^{\prime}}, i^{\prime} \neq i, m^{\prime} \geq l$, autre que $b_{i_{1}}^{-1}$ dans le cas où $\sigma_{i}^{l-1}=\sigma_{i_{1}}^{0}$;

- $\partial b_{i}^{l-1}$ contient $\left.\Gamma_{i}\right|_{\left[t_{i}^{l+1}-\eta_{i}^{l-1}, t_{i}^{l-1}+\eta_{i}^{l-1}\right]}$ si $\sigma_{i}^{l-1} \notin O_{i}$.

Puisque par hypothèse, tout arc attache $\eta_{i}^{l^{\prime}}, l^{\prime}<l-1$ qui rencontre $\left.\Gamma_{i}\right|_{\left[_{i}^{l-1}, t_{i}^{\prime \prime l}{ }_{i}\right]}$ est disjoint de toute brique $b_{i}^{m}, m \geq l$, on peut supposer de plus que la condition (x) est également remplie.

On peut vérifier que les conditions du lemme sont maintenant vérifiées jusqu'à l'ordre $l-1$. Nous avons dessiné les disques $b_{i}^{l}, i \in \mathbb{Z} / n \mathbb{Z}, l<0$, sur la Figure 12 .

Remarque Écrivons $\sigma_{i}^{*}=\sigma_{i}^{l_{i}}$. Puisque les $V_{i}^{l}$ peuvent être choisis arbitrairement proches des $\alpha_{i}^{l}$ et puisque $\left.\Gamma_{i}\right|_{\left[\left[t_{i}^{0}\right], t_{i}^{0}\right]}$ est libre, on peut supposer que $\bigcup_{l_{i} \leq l<0} b_{i}^{l_{i}}$ (qui est un disque fermé) est libre.

\section{Construction de la famille $\left(b_{i}^{\prime l}\right)_{i \in \mathbb{Z} / n \mathbb{Z}, l<0}$}

On va modifier de façon naturelle la famille $\left(b_{i}^{l}\right)_{i \in \mathbb{Z} / n \mathbb{Z}, l<0}$. On définit, pour tout $r<0$, une famille $\left(b_{i, r}^{l}\right)_{i \in \mathbb{Z} / n \mathbb{Z}, l<0}$ de disques libres par les relations de récurrence suivantes, où $m[l] \in\{l+1, \ldots,-1\}$ est défini par l'assertion (viii) du Lemme 7.2:

- $b_{i,-1}^{l}=b_{i}^{l}$, pour tout $l<0$;

- $b_{i, r}^{l}=b_{i}^{l}$, pour tout $l<r$; 


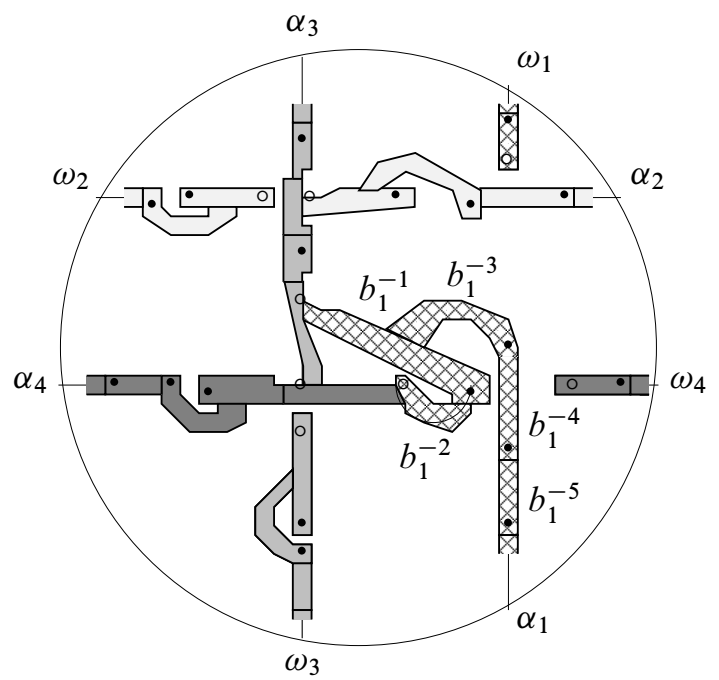

Figure 12

- $\quad$ si $b_{i}^{r} \cup b_{i, r+1}^{m[r]}$ n'est pas libre, on pose

$$
b_{i, r}^{l}=b_{i, r+1}^{l}, \text { pour tout } l<0 ;
$$

si $b_{i}^{r} \cup b_{i, r+1}^{m[r]}$ est libre, on pose

$$
\begin{cases}b_{i, r}^{r}=b_{i}^{r} \cup b_{i, r+1}^{m[r]}, & \\ b_{i, r}^{l}=b_{i}^{r} \cup b_{i, r+1}^{m[r]}, & \text { si } l>r \text { et } b_{i, r+1}^{l}=b_{i, r+1}^{m[r]}, \\ b_{i, r}^{l}=b_{i, r+1}^{l}, & \text { si } l>r \text { et } b_{i, r+1}^{l} \neq b_{i, r+1}^{m[r]} .\end{cases}
$$

Chaque disque $b_{i, r}^{l}$ est une réunion de disques $b_{i}^{m}, m \geq l$, et contient $b_{i}^{l}$. Puisque $b_{i}^{l}$ est inclus dans le disque de sécurité $V_{i}^{l}$ et puisque $f\left(V_{i}^{m}\right) \cap V_{i}^{l}=\varnothing$, si $m>l$, on peut remarquer, dans la construction précédente que $f\left(b_{i}^{r}\right) \cap b_{i, r+1}^{m[r]} \neq \varnothing$, dans le cas où $b_{i}^{r} \cup b_{i, r+1}^{m[r]}$ n'est pas libre. On en déduit:

Lemme 7.4 Pour tout $r<-1$ et tout $l \in\{r, \ldots,-2\}$, il existe $m \in\{l+1, \ldots,-1\}$ tel que $b_{i, r}^{\prime l}=b_{i, r}^{\prime m}$ ou $f\left(b_{i, r}^{\prime l}\right) \cap b_{i, r}^{\prime m} \neq \varnothing$.

Remarquons maintenant:

Lemme 7.5 Deux cas sont possibles: (i) $f$ est récurrent;

(ii) tout disque $b_{i, r}^{l}$ contient au plus $n-1$ disques $b_{i}^{m}$. 
Démonstration Chaque disque $b_{i}^{m}, m<0$, contient $\sigma_{i}^{m}$. Parmi ces singularités, il y en a au plus $n-1$ qui sont l'extrémité d'un graphe $A_{i^{\prime}}^{-}, i^{\prime} \neq i$. Ainsi un disque $b_{i, r}^{l}$ qui contient au moins $n$ disques $b_{i}^{m}$ contient deux points de l'orbite $O_{i}$ et rencontre donc l'un de ses itérés. Puisque $b_{i, r}^{l}$ est libre, $f$ est nécessairement récurrent, d'après la Proposition 1.4.

Nous supposerons dorénavant que tout disque $b_{i, r}^{l}$ contient au plus $n-1$ disques $b_{i}^{m}$. La suite $\left(b_{i, r}^{l}\right)_{r<0}$ est alors stationnaire. On définit donc une famille de disques $\left(b_{i}^{\prime l}\right)_{i \in \mathbb{Z} / n \mathbb{Z}, l<0}$ en notant $b_{i}^{\prime l}$ la valeur de $b_{i, r}^{l}$, pour $r$ assez petit. Nous avons dessiné les disques $b_{i}^{\prime l}, i \in \mathbb{Z} / n \mathbb{Z}, l<0$, sur la Figure 13 .

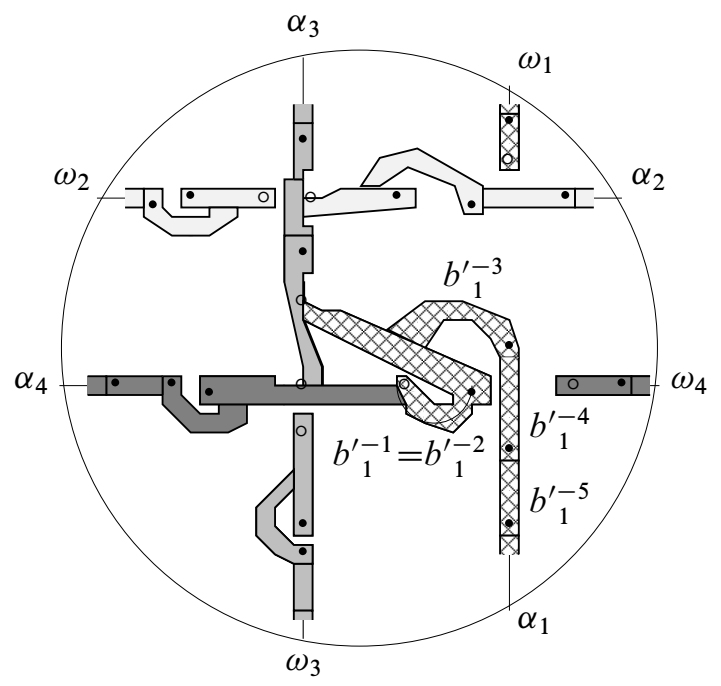

Figure 13

La remarque qui suit le Lemme 7.3 implique le résultat suivant:

Lemme 7.6 Si on écrit $\sigma_{i}^{*}=\sigma_{i}^{l_{i}}$, les briques $b_{i}^{\prime m}, l_{i} \leq m<0$, sont toutes égales. En particulier, $b_{i}^{\prime-1}$ contient $\sigma_{i}^{*}$.

Grâce au Lemme 7.4, on sait que pour tout $l<-1$, il existe $m \in\{l+1, \ldots,-1\}$ tel que $b_{i}^{\prime l}=b_{i}^{\prime m}$ ou $f\left(b_{i}^{\prime l}\right) \cap b_{i}^{\prime m} \neq \varnothing$. On en déduit:

Lemme 7.7 Supposons que $l<-1$ et que ${b^{\prime}}_{i}^{l} \neq b_{i}^{-1}$. Il existe alors une chaîne de disques choisis dans les $b_{i}^{\prime l}, l \leq m<0$, qui joint $b_{i}^{\prime l}$ à $b_{i}^{\prime-1}$.

Utilisant maintenant le fait que chaque $b_{i}^{\prime l}, l>0$, contient $\sigma_{i}^{l+1}$ (qui est un point de $O_{i}$ ), le fait que $b_{i}^{\prime-1}$ contient $\sigma_{i}^{*}$ et le résultat que l'on vient de noter, on obtient: 
Lemme 7.8 Soient $l$ et $l^{\prime}$ deux entiers non nuls tels que $l<l^{\prime}$. Il existe une chaîne de disques choisis dans les ${b^{\prime}}_{i}^{m}, l \leq m \leq l^{\prime}$, qui joint ${b^{\prime}}_{i}^{l}$ à $b_{i}^{\prime l^{\prime}}$ dans les cas suivants:

- $l<l^{\prime}=-1$ et $b_{i}^{\prime l} \neq b_{i}^{\prime-1}$;

- $l<0<l^{\prime}$

- $0<l<l^{\prime}$.

\section{Construction de la famille $\left(b^{\prime \prime l}\right)_{i \in \mathbb{Z} / n \mathbb{Z}, l \neq 0}$}

On peut remarquer que l'ensemble $\bigcup_{i \in \mathbb{Z} / n \mathbb{Z}, l \neq 0} \partial b_{i}^{\prime l}$ est le squelette d'une décomposition en briques $\mathcal{D}^{\prime}$ de $\mathbf{D} \backslash \operatorname{Fix}(f)$ dont les $b_{i}^{\prime l}$ sont des briques libres. On peut décomposer les briques de $\mathcal{D}^{\prime}$ qui ne sont pas libres pour obtenir une décomposition libre de $\mathbf{D} \backslash \operatorname{Fix}(f)$. On peut ensuite considèrer une sous-décomposition libre maximale $\mathcal{D}^{\prime \prime}=\left(S^{\prime \prime}, A^{\prime \prime}, B^{\prime \prime}\right)$ de cette décomposition. Chaque disque $b_{i}^{\prime l}$ est alors contenu dans une brique $b_{i}^{\prime \prime l}$. Il faut noter qu'on peut avoir $b_{i}^{\prime \prime l}=b_{i^{\prime}}^{\prime \prime l^{\prime}}$ même si $b_{i}^{\prime l} \neq b_{i^{\prime}}^{\prime l^{\prime}}$ et même si $i \neq i^{\prime}$. Nous avons dessiné les disques $b_{i}^{\prime \prime \prime}, i \in \mathbb{Z} / n \mathbb{Z}, l \neq 0$, sur la Figure 14 .

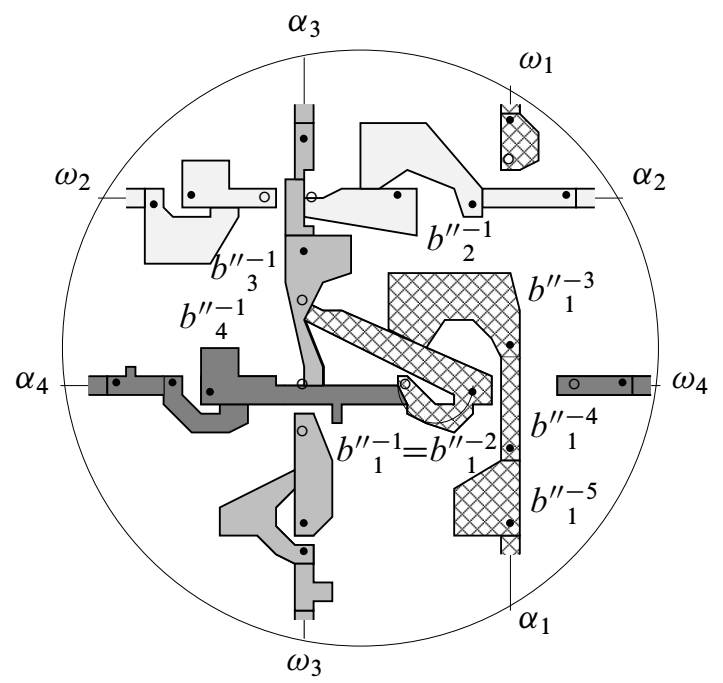

Figure 14

\section{Démonstration du caractère récurrent de $f$}

Nous allons prouver dans cette section que $f$ est récurrent en construisant une chaîne fermée de briques de $\mathcal{D}^{\prime \prime}$. En d'autres termes, nous allons trouver une brique $b^{\prime \prime} \in$ $B^{\prime \prime}$ qui appartient à son futur strict, c'est-à-dire une brique $b^{\prime \prime}$ telle que $b^{\prime \prime} \in b_{>}^{\prime \prime}$. 
Rassemblons dans une proposition les propriétés que nous allons utiliser. On peut supposer que la première est vérifiée, d'après la Proposition 2.2. Les autres se déduisent des résultats de la section précédente et du fait que $b^{\prime \prime l}{ }_{i}$ contient $b_{i}^{\prime l}$.

Proposition 8.1 Nous avons les résultats suivants:

(i) pour tout $b^{\prime \prime} \in B^{\prime \prime}$, les ensembles $b^{\prime \prime} \geq, b^{\prime \prime} \leq, b^{\prime \prime}>$ et $b^{\prime \prime}<$ sont connexes;

(ii) $b_{i}^{\prime \prime \prime} l_{i}^{\prime} \in\left(b_{i}^{\prime \prime l}\right)_{\geq}$, si $0<l<l^{\prime}$, si $l<l^{\prime}=-1$ ou si $l<0<l^{\prime}$;

(iii) $\sigma_{i}^{l+1} \in \operatorname{Int}\left(b_{i}^{\prime \prime l}\right)$ si $l>0$;

(iv) $\sigma_{i}^{l} \in b_{i}^{\prime \prime l}$ si $l<0$;

(v) $\sigma_{i}^{\prime \prime l} \in \operatorname{Int}\left(b_{i}^{\prime \prime l}\right)$, si $\sigma_{i}^{l} \in O_{i}$ et $l<0$;

(vi) $\sigma_{i}^{*} \in \operatorname{Int}\left(b_{i}^{\prime \prime-1}\right)$.

Nous prouverons donc:

Proposition 8.2 Il existe $i \in \mathbb{Z} / n \mathbb{Z}$ tel que $b_{i}^{\prime \prime-1} \in\left(b_{i}^{\prime \prime-1}\right)_{>}$.

Démonstration Fixons $i \in \mathbb{Z} / n \mathbb{Z}$. On sait, d'après la Proposition 6.2, qu'il existe $i^{\prime} \notin\{i, i+1\}$ tel que $A_{i+1}^{-} \cap A_{i^{\prime}}^{-} \neq \varnothing$ et que $A_{i+1}^{-} \cup A_{i^{\prime}}^{-}$est donc connexe. Par construction des briques $b_{i}^{l}, b_{i}^{\prime l}$ et $b_{i}^{\prime \prime l}$, on sait que

$$
A_{i+1}^{-} \cup A_{i^{\prime}}^{-} \subset X=\left(\bigcup_{l<0} b^{\prime \prime l}{ }_{i+1}\right) \cup\left(\bigcup_{l<0} b^{\prime \prime l}{ }_{i^{\prime}}\right) .
$$

On sait de plus que $X$ est connexe comme réunion de briques qui rencontrent $A_{i+1}^{-} \cup$ $A_{i^{\prime}}^{-}$. Nous allons montrer que le futur $\left(b^{\prime \prime}{ }_{i}^{-1}\right) \geq$ de $b_{i}^{\prime \prime}{ }_{i}^{-1}$ contient au moins une brique qui est dans $X$. On sait, d'après la Proposition 6.2, que les points $\sigma_{i}^{l}, l>0$, sont séparés de $\sigma_{i}^{*}$ par $A_{i+1}^{-} \cup A_{i^{\prime}}^{-}$. Puisque $\left(b_{i}^{\prime \prime}-1\right) \geq$ est connexe, la partie Int $\left(\left(b_{i}^{\prime \prime}-1\right) \geq\right) \subset \mathbf{D}$ est également connexe. Puisque la partie Int $\left(\left(b^{\prime \prime}{ }_{i}^{-1}\right)_{\geq}\right)$contient les points $\sigma_{i}^{l}, l>0$, ainsi que le point $\sigma_{i}^{*}$, elle rencontre $A_{i+1}^{-} \cup A_{i^{\prime}}^{-}$. Ceci implique que l'une au moins des briques de $\left(b^{\prime \prime}{ }_{i}^{-1}\right) \geq$ est une brique de $X$. En utilisant l'assertion (ii) de la Proposition 8.2 , on en déduit que le futur de $b^{\prime \prime}{ }_{i}^{-1}$ contient $b_{i+1}^{\prime \prime-1}$ ou $b^{\prime \prime} i^{\prime}$. En conclusion, on sait que pour tout $i \in \mathbb{Z} / n \mathbb{Z}$, il existe $i^{\prime} \neq i$ tel que $b_{i^{\prime}}^{\prime \prime-1} \in\left(b^{\prime \prime}-1\right) \geq$.

Il vaut maintenant envisager deux cas:

- les briques $b^{\prime \prime}-1, i \in \mathbb{Z} / n \mathbb{Z}$, sont toutes distinctes;

- il existe $i_{0}$ et $i_{1}$ distincts tels que $b^{\prime \prime-1} i_{0}=b^{\prime \prime-1} i_{1}$. 
Dans le premier cas, le fait que pour tout $i \in \mathbb{Z} / n \mathbb{Z}$, il existe $i^{\prime} \neq i$ tel que $b^{\prime \prime-1} \in$ $\left(b^{\prime \prime}-1\right)_{i}$ implique qu'il existe $i \in \mathbb{Z} / n \mathbb{Z}$ tel que $b_{i}^{\prime \prime-1} \in\left(b_{i}^{\prime \prime-1}\right)_{>}$, la proposition est démontrée.

Plaçons nous maintenant dans le second cas et supposons qu'il existe $i_{0}$ et $i_{1}$ distincts tels que $b^{\prime \prime} i_{0}=b^{\prime \prime}{ }_{i_{1}}$. Nous allons prouver que $b^{\prime \prime} \in b_{>}^{\prime \prime}$, où $b^{\prime \prime}=b^{\prime \prime} i_{0}=b^{\prime \prime}{ }_{i_{1}}$. On peut bien sûr supposer que $b^{\prime \prime}$ ne rencontre aucun de ses itérés stricts et rencontre donc toute orbite $O_{i}$ en au plus un point. Nous avons illustré cette situation sur la Figure 15.

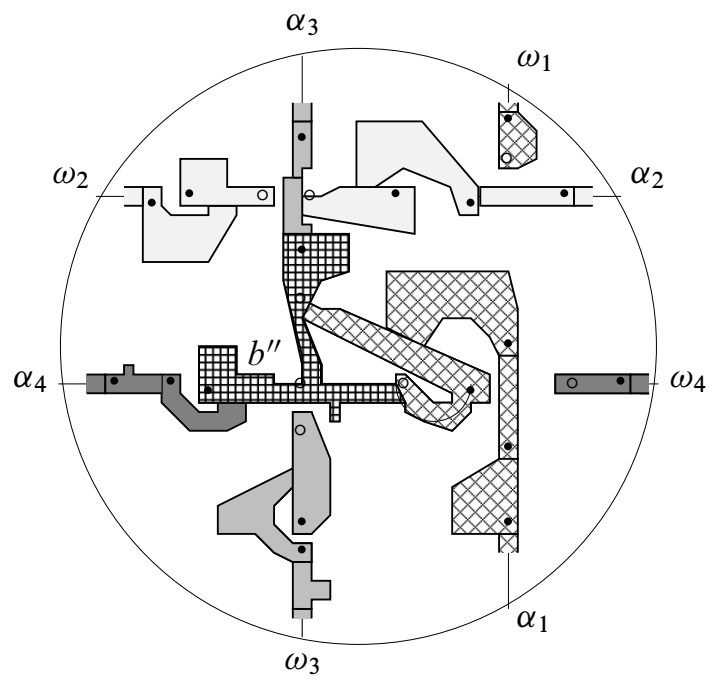

Figure 15

Commençons par prouver que pour tout $i \in \mathbb{Z} / n \mathbb{Z}$, il existe $l_{i}<0$ tel que $b^{\prime \prime \prime}{ }_{i} \in b_{<}^{\prime \prime}$ si $l \leq l_{i}$. Pour cela, notons $I$ l'ensemble des indices satisfaisant cette condition. Nous savons déjà que $b_{i}^{\prime \prime l} \neq b^{\prime \prime}$ pour $l$ petit puisque la singularité $\sigma_{i}^{l}$ (qui est dans $b^{\prime \prime l}{ }_{i}$ ) appartient alors à $O_{i}$. Remarquons que $I$ contient $i_{0}$ et $i_{1}$. En effet, on sait que les briques ${b^{\prime \prime}}_{i_{0}}^{\prime \prime}$ et $b^{\prime \prime l}{ }_{i_{1}}$ appartiennent à $\left(b^{\prime \prime-1}\right)_{\leq}$et qu'elles sont distinctes de $b^{\prime \prime-1}$ si $l$ est petit. Pour prouver que $I=\mathbb{Z} / n \mathbb{Z}$, il suffit donc de montrer que $i-1 \in I$ dès que $i \in I$. Posons $i^{\prime}=i_{1}$ si $i \neq i_{1}$ et $i^{\prime}=i_{2}$ si $i=i_{1}$. Raisonnons par l'absurde et supposons que $i-1 \notin I$. Puisque $b_{i-1}^{\prime \prime l} \in\left(b_{i-1}^{\prime \prime} l_{i}^{\prime}\right)_{\leq}$, si $l<0<l^{\prime}$, on en déduit que $b_{<}^{\prime \prime}$ ne contient aucune brique $b_{i-1}^{\prime \prime} l^{\prime}, l^{\prime}>0$. Puisque $b^{\prime \prime}<$ est connexe (assertion (i) de la Proposition 8.1) et contient toutes les briques $b_{i}^{\prime \prime l}$ et $b^{\prime \prime}{ }_{i^{\prime}}$, pour $l<0$ assez petit, l'ensemble $b^{\prime \prime}<$ sépare les points $\sigma_{i-1}^{-l}$ et $\sigma_{i-1}^{l+1}$, dès que $l$ est assez grand. Or le futur $\left(b^{\prime \prime}{ }_{i-1}^{-l}\right)_{\geq}$est connexe et contient $b_{i-1}^{\prime \prime l}$. La partie $\operatorname{Int}\left(\left(b_{i-1}^{\prime \prime}-l\right) \geq\right) \subset \mathbf{D}$ est connexe, elle contient $\sigma_{i-1}^{-l}$ et $\sigma_{i-1}^{l+1}$, et rencontre donc l'ensemble $b_{<}^{\prime \prime} \subset \mathbf{D}$. Les parties 
$\left(b^{\prime \prime}-l-1\right)_{\geq}$et $b_{<}^{\prime \prime}$ de $B^{\prime \prime}$ ont donc au moins une brique en commun, ce qui implique que $b_{i-1}^{\prime \prime}-l \in b_{<}^{\prime \prime}$. Ainsi $i-1 \in I$, ce qui contredit l'hypothèse.

Un argument très proche permet alors de montrer que $b^{\prime \prime} \in b^{\prime \prime}>$. Là encore, raisonnons par l'absurde et supposons que $b^{\prime \prime}<$ et $b^{\prime \prime} \geq$ n'ont aucune brique en commun. Utilisant de nouveau la connexité de $b^{\prime \prime}<$ et le fait que $I=\mathbb{Z} / n \mathbb{Z}$, on peut remarquer que si $l$ est grand, $b^{\prime \prime \prime} i_{0}$ et $b^{\prime \prime l}{ }_{i_{1}}$, qui sont des briques de $b^{\prime \prime} \geq$ et donc qui n'appartiennent pas à $b^{\prime \prime}<$, sont alors dans des composantes connexes différentes de $B^{\prime \prime} \backslash\left(b^{\prime \prime}<\right)$. Ceci est impossible puisque $b^{\prime \prime} \geq$ est connexe, contient $b^{\prime \prime \prime}{ }_{i_{0}}$ et $b^{\prime \prime l}{ }_{i_{1}}$ et n'a aucune brique en commun avec $b^{\prime \prime}<$.

\section{References}

[1] L E J Brouwer, Beweis des ebenen Translationssatzes, Math. Ann. 72 (1912) 37-54 MR1511684

[2] M Brown, A new proof of Brouwer's lemma on translation arcs, Houston J. Math. 10 (1984) 35-41 MR736573

[3] M Brown, J M Kister, Invariance of complementary domains of a fixed point set, Proc. Amer. Math. Soc. 91 (1984) 503-504 MR744656

[4] A Fathi, An orbit closing proof of Brouwer's lemma on translation arcs, Enseign. Math. (2) 33 (1987) 315-322 MR925994

[5] A Floer, Proof of the Arnol'd conjecture for surfaces and generalizations to certain Kähler manifolds, Duke Math. J. 53 (1986) 1-32 MR835793

[6] M Flucher, Fixed points of measure preserving torus homeomorphisms, Manuscripta Math. 68 (1990) 271-293 MR1065931

[7] J Franks, Generalizations of the Poincaré-Birkhoff theorem, Ann. of Math. (2) 128 (1988) 139-151 MR951509

[8] J Franks, Area preserving homeomorphisms of open surfaces of genus zero, New York J. Math. 2 (1996) 1-19 MR1371312

[9] J Franks, Rotation vectors and fixed points of area preserving surface diffeomorphisms, Trans. Amer. Math. Soc. 348 (1996) 2637-2662 MR1325916

[10] L Guillou, Théorème de translation plane de Brouwer et généralisations du théorème de Poincaré-Birkhoff, Topology 33 (1994) 331-351 MR1273787

[11] M Handel, A fixed-point theorem for planar homeomorphisms, Topology 38 (1999) 235-264 MR1660349

[12] M Handel, W P Thurston, New proofs of some results of Nielsen, Adv. in Math. 56 (1985) 173-191 MR788938 
[13] T Homma, An extension of the Jordan curve theorem, Yokohama Math. J. 1 (1953) 125-129 MR0058194

[14] P Le Calvez, Une version feuilletée équivariante du théorème de translation de Brouwer, Publ. Math. Inst. Hautes Études Sci. (2005) 1-98 MR2217051

[15] F Le Roux, Homéomorphismes de surfaces: théorèmes de la fleur de Leau-Fatou et de la variété stable, Astérisque (2004) vi+210 MR2068866

[16] S Matsumoto, Arnold conjecture for surface homeomorphisms, from: "Proceedings of the French-Japanese Conference: Hyperspace Topologies and Applications (La Bussière, 1997)", Topology Appl. 104 (2000) 191-214 MR1780985

[17] A Sauzet, Application des décompositions libres à l'étude des homéomorphismes de surface Thèse de l'Université Paris 13 (2001)

[18] S Schwartzman, Asymptotic cycles, Ann. of Math. (2) 66 (1957) 270-284 MR0088720

[19] J-C Sikorav, Points fixes d'une application symplectique homologue à l'identité, J. Differential Geom. 22 (1985) 49-79 MR826424

Laboratoire Analyse, Géométrie et Applications, C.N.R.S.-U.M.R 7539, Institut Galilée Université Paris 13, Avenue J.-B.Clément, 93430 Villetaneuse, France

lecalvez@math.univ-paris13.fr

Proposed: Leonid Polterovich

Seconded: Yasha Eliashberg, Benson Farb
Received: 1 June 2006 Accepted: 28 October 2006 